\title{
Synthesis and peptide binding properties of methoxypyrrole amino acids (MOPAS)
}

Christoph Bonauer, Manfred Zabel ${ }^{\S}$ and Burkhard König*

Institut für Organische Chemie, Universität Regensburg,D-93040

Regensburg, Germany

\section{SUPPORTING INFORMATION}

page

1. Experimental procedures and compound characterisation 2

2. X-ray crystal structures 12

3. Details of NMR titrations 58

4. NMR data for structure determination 68

5. Copies of ${ }^{1} \mathrm{H}$ NMR spectra $\quad 75$ 


\section{Experimental procedures and compound characterization}

Ethyl 5-formyl-3-methoxy-4-methyl-1H-pyrrole-2-carboxylate (3): A solution of $4.55 \mathrm{~g}$ (24.8 mmol) of ethyl 3-methoxy-4-methyl-1H-pyrrole-2-carboxylate (2) in $50 \mathrm{~mL}$ of 1,2-dichlorethane was added drop wise to an ice cold solution of dimethylformamide $(1.81 \mathrm{~g}$, $24.8 \mathrm{mmol}$ ) in $50 \mathrm{~mL}$ of 1,2-dichlorethane containing $\mathrm{POCl}_{3}(3.81 \mathrm{~g}, 24.8 \mathrm{mmol})$. After stirring for $1 \mathrm{~h}$ at room temp. the mixture was heated to reflux for $5 \mathrm{~h}$, then cooled to room temp., $150 \mathrm{~mL}$ of EtOAc and $200 \mathrm{~mL}$ of $\mathrm{H}_{2} \mathrm{O}$ were added and the organic layer was separated. The aqueous layer was washed twice with $100 \mathrm{~mL}$ of ether and the combined organic layers were washed five times with $400 \mathrm{~mL}$ of $10 \%$ aqueous $\mathrm{Na}_{2} \mathrm{CO}_{3}$. Evaporation of the solvent gave $5.20 \mathrm{~g}$ of crude product which was purified by column chromatography on silica gel (PE : EtOAc, 70:30). Yield: $5.01 \mathrm{~g}(23.6 \mathrm{mmol}, 95 \%)$ of 3. $R_{\mathrm{f}}=0.29$. M.p. $=95.5-96{ }^{\circ} \mathrm{C}$. ${ }^{1} \mathrm{H}$ NMR (250 MHz, $\mathrm{CDCl}_{3}$ ): $\delta=1.39$ (t, $\left.J=7.13 \mathrm{~Hz}, 3 \mathrm{H}\right), 2.29$ (s, $\left.3 \mathrm{H}\right), 3.89$ (s, $\left.3 \mathrm{H}\right), 4.35$ (q, $J=7.13 \mathrm{~Hz}, 2 \mathrm{H}), 9.22$ (bs, $1 \mathrm{H}), 9.74$ (bs, $1 \mathrm{H}) .{ }^{13} \mathrm{C} \mathrm{NMR}\left(62 \mathrm{MHz}, \mathrm{CDCl}_{3}\right): \delta=6.81(+$, $1 \mathrm{C}), 14.34$ (+, $1 \mathrm{C}), 61.14(-, 1 \mathrm{C}), 62.48(+, 1 \mathrm{C}), 117.44\left(\mathrm{C}_{\text {quat }}, 1 \mathrm{C}\right), 122.30\left(\mathrm{C}_{\text {quat }}, 1 \mathrm{C}\right)$, $127.74\left(\mathrm{C}_{\text {quat }}, \quad 1 \mathrm{C}\right), \quad 150.29\left(\mathrm{C}_{\text {quat }}, \quad 1 \mathrm{C}\right), 159.59 \quad\left(\mathrm{C}_{\text {quat }}, 1 \mathrm{C}\right), 179.12 \quad(+, \quad 1 \mathrm{C})$. IR (KBr): $\tilde{v}=3273 \mathrm{~cm}^{-1}$ (s), $2991(\mathrm{w}), 2819$ (w), 1676 (s), 1557 (m), 1515 (s), 1489 (s), 1139 (s). MS (EI, $70 \mathrm{eV}): m / z(\%)=211(100)\left[\mathrm{M}^{+}\right], 164(54.32)\left[\mathrm{M}^{+}-\mathrm{C}_{2} \mathrm{H}_{6} \mathrm{O}\right], 150$ (43.06) $\left[\mathrm{M}^{+}-\right.$ $\mathrm{C}_{3} \mathrm{H}_{9} \mathrm{O}$ ]. Anal. calcd. for $\mathrm{C}_{10} \mathrm{H}_{13} \mathrm{NO}_{4}$ : C, 56.87; $\mathrm{H}, 6.20 ; \mathrm{N}, 6.63$, found C, 56.79; $\mathrm{H}, 6.05$; $\mathrm{N}, 6.58$.

\section{Ethyl 5-[tert-butoxycarbonylamino-(toluene-4-sulfonyl)-methyl]-3-methoxy-4-methyl-}

1H-pyrrole-2-carboxylate (4): A mixture of $t$-butylcarbamate $(1.1 \mathrm{~g}, 9.42 \mathrm{mmol}), 3.80 \mathrm{~mL}$ of THF, $4.00 \mathrm{~mL}$ of $\mathrm{H}_{2} \mathrm{O}$, sodium $p$-toluenesulfinate ( $\left.1.68 \mathrm{~g}, 9.42 \mathrm{mmol}\right)$, aldehyde 3 ( $2 \mathrm{~g}$, $9.42 \mathrm{mmol})$ and formic acid $(2.34 \mathrm{~mL}, 2.85 \mathrm{~g}, 62.0 \mathrm{mmol})$ was stirred until it became homogeneous. After addition of additional $\mathrm{H}_{2} \mathrm{O}(4.5 \mathrm{~mL})$, the mixture was stirred for $6 \mathrm{~d}$ at room temp. The crystalline sulfone $\mathbf{4}$ was filtered off with suction, washed successively with water and $\mathrm{PE}$ and dried over $\mathrm{P}_{2} \mathrm{O}_{5}$. Sulfone 4 was used without further purification for subsequent steps. ${ }^{1} \mathrm{H}$ NMR $\left(250 \mathrm{MHz}, \mathrm{CDCl}_{3}\right): \delta=1.31(\mathrm{~s}, 9 \mathrm{H}), 1.42(\mathrm{t}, J=6.8 \mathrm{~Hz}, 3 \mathrm{H}), 1.78(\mathrm{~s}, 3 \mathrm{H})$, 2.39 (s, $3 \mathrm{H}), 3.83$ (s, $3 \mathrm{H}), 4.46$ (q, $J=6.88 \mathrm{~Hz}, 2 \mathrm{H}), 6.00$ (bd, $J=10.7 \mathrm{~Hz}, 1 \mathrm{H}), 6.92$ (bd, $J=10.7 \mathrm{~Hz}, 1 \mathrm{H}), 7.28(\mathrm{~m}, 2 \mathrm{H}), 7.68$ (m, $2 \mathrm{H}), 10.05$ (bs, $1 \mathrm{H})$. - IR (KBr): $\widetilde{v}=3330 \mathrm{~cm}^{-1}$ (s), 2979 (m), 2932 (sh), 1717 (s), 1654 (s), 151529 (S), 1474 (s). 


\section{Boc-MOPAS-OEt， (Ethyl 5-(tert-butoxycarbonylamino-methyl)-3-methoxy-4-methyl-}

1H-pyrrole-2-carboxylate) (5): To a suspension of $\mathrm{NaBH}_{4}(433 \mathrm{mg}, 11.4 \mathrm{mmol})$ in $20 \mathrm{~mL}$ of THF, solid crude $\alpha$-amidoalkylsulfone 4 (2.59 g, $5.55 \mathrm{mmol})$ was added portion wise while the mixture was ice cooled. Stirring was continued for 15 min with ice cooling and $2 \mathrm{~h}$ at room temp. The mixture was ice cooled again, quenched with $5 \mathrm{~mL}$ of satd. aqueous $\mathrm{NH}_{4} \mathrm{Cl}$, stirring was continued for $30 \mathrm{~min}$, the organic layer was separated and the aqueous phase was extracted twice with $50 \mathrm{~mL}$ of $\mathrm{CH}_{2} \mathrm{Cl}_{2}$. The combined organic layers were dried over $\mathrm{MgSO}_{4}$ and evaporated to give $1.73 \mathrm{~g}$ (5.54 mmol, quant.) of the 5, m.p. $=114-115{ }^{\circ} \mathrm{C}$. ${ }^{1} \mathrm{H}$ NMR (250 MHz, $\left.\mathrm{CDCl}_{3}\right): \delta=1.35(\mathrm{t}, J=7.13 \mathrm{~Hz}, 3 \mathrm{H}), 1.46(\mathrm{~s}, 9 \mathrm{H}), 1.95$ (s, $\left.3 \mathrm{H}\right), 3.86$ $(\mathrm{s}, 3 \mathrm{H}), 4.16(\mathrm{~d}, J=5.94 \mathrm{~Hz}, 2 \mathrm{H}), 4.31$ (q, $J=7.13 \mathrm{~Hz}, 2 \mathrm{H}), 4.96$ (bs, $1 \mathrm{H}), 8.97$ (bs, $1 \mathrm{H})$. ${ }^{13} \mathrm{C} \mathrm{NMR}\left(62 \mathrm{MHz}, \mathrm{CDCl}_{3}\right): \delta=7.02(+, 1 \mathrm{C}), 14.47$ (+, $\left.1 \mathrm{C}\right), 28.37$ (+, $\left.1 \mathrm{C}\right), 35.89(-, 1 \mathrm{C})$, 60.03 (-, $1 \mathrm{C}), 62.16(+, 1 \mathrm{C}), 80.04\left(\mathrm{C}_{\text {quat }}, 1 \mathrm{C}\right), 110.06\left(\mathrm{C}_{\text {quat }}, 1 \mathrm{C}\right), 110.21\left(\mathrm{C}_{\text {quat }}, 1 \mathrm{C}\right)$, $129.68\left(\mathrm{C}_{\text {quat }}, 1 \mathrm{C}\right), 151.09$ ( $\left.\mathrm{C}_{\text {quat }}, 1 \mathrm{C}\right), 156.51\left(\mathrm{C}_{\text {quat }}, 1 \mathrm{C}\right), 160.48\left(\mathrm{C}_{\text {quat }}, 1 \mathrm{C}\right) . \mathrm{MS}(\mathrm{EI}, 70$ $\mathrm{eV}): m / z(\%)=312(33.53)\left[\mathrm{M}^{+}\right], 256(64.17)\left[\mathrm{M}^{+}-\mathrm{C}_{4} \mathrm{H}_{8}\right], 255$ (33.80) $\left[\mathrm{M}^{+}-\mathrm{C}_{4} \mathrm{H}_{9}\right], 227(100)$, 165 (91.35). IR (KBr): $\widetilde{v}=3332 \mathrm{~cm}^{-1}$ (s), 3296 (s), 3059 (w), 2983 (m), 2936 (sh), 2870 (sh), 1686 (s), 1657 (s), 1538 (s), 1471 (s). Anal. calcd. for $\mathrm{C}_{15} \mathrm{H}_{24} \mathrm{~N}_{2} \mathrm{O}_{5}$ : C, 57.68; H, 17.74; $\mathrm{N}, 8.97$, found $\mathrm{C}, 57.64 ; \mathrm{H}, 7.68 ; \mathrm{N}, 8.97$.

\section{Boc-MOPAS-OH (5-(tert-Butoxycarbonylamino-methyl)-3-methoxy-4-methyl-1H-pyr-}

role-2-carboxylic acid) (6): A solution of $1.58 \mathrm{~g}(5.06 \mathrm{mmol})$ of 5 in $20 \mathrm{~mL}$ of $\mathrm{MeOH}$ and $20 \mathrm{~mL}$ of $2 \mathrm{~N} \mathrm{NaOH}$ was heated under reflux for $2.5 \mathrm{~h}$ and cooled to room temperature. The solution was washed twice with $30 \mathrm{~mL}$ of $\mathrm{CH}_{2} \mathrm{Cl}_{2}$ and the aqueous layer was acidified with 1 $\mathrm{N} \mathrm{H}_{2} \mathrm{SO}_{4}$ to $\mathrm{pH}=2$. The precipitate was collected with suction and washed with $\mathrm{H}_{2} \mathrm{O}$. M.p. = $102-103{ }^{\circ} \mathrm{C} .{ }^{1} \mathrm{H}$ NMR $\left(250 \mathrm{MHz}, \mathrm{CDCl}_{3}\right): \delta=1.46$ (s, $\left.9 \mathrm{H}\right), 2.00$ (s, $\left.3 \mathrm{H}\right), 3.92$ (s, $\left.3 \mathrm{H}\right), 4.21$ (d, $J=5.93 \mathrm{~Hz}, 2 \mathrm{H}), 5.15$ (bs, $1 \mathrm{H}), 9.53$ (bs, $1 \mathrm{H}) . \mathrm{IR}(\mathrm{KBr}): \tilde{v}=3351 \mathrm{~cm}^{-1}(\mathrm{~m}), 3261$ (sh), $2980(\mathrm{w}), 1688$ (s), $1656(\mathrm{~m}), 1584$ (w), 1513 (m), 1487 (m).

\section{Boc-MOPAS-OBt (Benzotriazol-1-yl-5-(tert-butoxycarbonylamino-methyl)-3-methoxy-} 4-methyl-1H-pyrrole-2-carboxylate) (7): To a solution of $6(286 \mathrm{mg}, 1.01 \mathrm{mmol})$ in $6 \mathrm{~mL}$ of DMF, HOBt (136 mg, $1.01 \mathrm{mmol})$ and DCC (208 mg, $1.01 \mathrm{mmol})$ were added and the mixture stirred for $40 \mathrm{~h}$ at room temp.. The reaction mixture was filtrated drop wise into 60 $\mathrm{mL}$ of ice water and left for $15 \mathrm{~min}$. The precipitate was collected with suction and dissolved in $20 \mathrm{~mL}$ of $\mathrm{CH}_{2} \mathrm{Cl}_{2}$. The organic layer was separated and added drop wise into $40 \mathrm{~mL}$ of ice cooled PE and left $4 \mathrm{~h}$ at $-20{ }^{\circ} \mathrm{C}$. The fine white powder was then collected with suction and 
dried in vacuum to yield $320 \mathrm{mg}(79 \%)$ 7, m.p. $>158{ }^{\circ} \mathrm{C}$ (decomp.). ${ }^{1} \mathrm{H}$ NMR (300 MHz, $\left.\mathrm{CDCl}_{3}\right): \delta=1.45(\mathrm{~s}, 9 \mathrm{H}), 2.02(\mathrm{~s}, 3 \mathrm{H}), 3.98$ (s, $\left.3 \mathrm{H}\right), 4.23(\mathrm{~d}, J=6.04 \mathrm{~Hz}, 2 \mathrm{H}), 5.18$ (bm, $1 \mathrm{H}), 7.39-8.10(\mathrm{~m}, 4 \mathrm{H}), 9.58(\mathrm{bs}, 1 \mathrm{H})$. MS (CI, NH $\mathrm{NH}_{3}: \mathrm{m} / z(\%)=241.2$ (90) $\left[\mathrm{MH}^{+}-\right.$ $\left.\mathrm{C}_{7} \mathrm{H}_{3} \mathrm{~N}_{3} \mathrm{O}_{2}\right], 284.2$ (28), $402.2(100)\left[\mathrm{MH}^{+}\right]$.

\section{Boc-D-Pro-Gly-Val-OMe (2-\{[(1-Methoxycarbonyl-2-methyl-propylcarbamoyl)-methyl]-} carbamoyl\}-pyrrolidine-1-carboxylic acid tert-butyl ester) (9): A solution of Boc-D-ProOH (8, $200 \mathrm{mg}, 734 \mu \mathrm{mol}), \mathrm{HOAt}(99.2 \mathrm{mg}, 734 \mu \mathrm{mol})$ and EDC (129 $\mu 1,114 \mathrm{mg}, 734 \mu \mathrm{mol})$ in $5 \mathrm{~mL}$ of $\mathrm{CH}_{2} \mathrm{Cl}_{2}$ was stirred for $15 \mathrm{~min}$. DIEA (128 $\left.\mu \mathrm{l}, 94.9 \mathrm{mg}, 734 \mu \mathrm{mol}\right)$ und $123 \mathrm{mg}$ (734 mmol) of $\mathrm{H}-\mathrm{Val}-\mathrm{OMe} \cdot \mathrm{HCl}$ were added and stirring was continued for $24 \mathrm{~h}$. After addition of $20 \mathrm{~mL}$ of $\mathrm{CH}_{2} \mathrm{Cl}_{2}$, the solution was washed twice with $10 \mathrm{~mL}$ of $5 \%$ aqueous $\mathrm{KHSO}_{4}$, once with $10 \mathrm{~mL}$ of $\mathrm{H}_{2} \mathrm{O}$ and twice with $10 \mathrm{~mL}$ of $0.5 \mathrm{~N}$ aqueous $\mathrm{NaHCO}_{3}$. The solution was dried over $\mathrm{MgSO}_{4}$, the solvent was evaporated and the crude product was purified by column chromatography $\left(\mathrm{SiO}_{2}, \mathrm{EtOAc}\right)$ to give $230 \mathrm{mg}(81 \%)$ of $\mathbf{9}, R_{\mathrm{f}}=0.24$. MS (ESI, $\left.\mathrm{CH}_{2} \mathrm{Cl}_{2} / \mathrm{MeOH}+10 \mathrm{mmol} / \mathrm{L} \mathrm{NH}_{4} \mathrm{Ac}\right): m / z(\%)=323(100), 386$ (86) $\left[\mathrm{MH}^{+}\right], 403$ (63) $\left[\mathrm{MNH}_{4}^{+}\right]$. Anal. calcd. for $\mathrm{C}_{18} \mathrm{H}_{31} \mathrm{~N}_{3} \mathrm{O}_{6}$ : C, 56.07; H, 8.11; N, 10.91; found C, 55.49; H, 8.60; N, 10.79 .

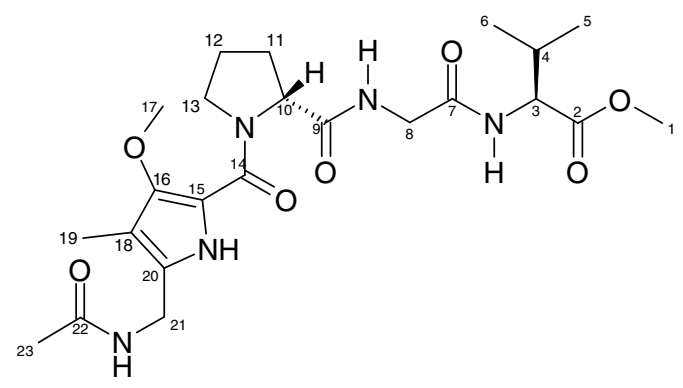

\section{Ac-MOPAS-D-Pro-Gly-Val-OMe (2-[2-(\{1-[5-(Acetylamino-methyl)-3-methoxy-4-meth-} yl-1H-pyrrole-2-carbonyl]-pyrrolidine-2-carbonyl\}-amino)-acetylamino]-4-methyl-pentanoic acid methyl ester) (10): A solution of 9 (195 mg, $506 \mu \mathrm{mol})$ in $4.5 \mathrm{ml}$ of $\mathrm{CH}_{2} \mathrm{Cl}_{2}$ containing 33\% TFA was stirred at room temperature for $20 \mathrm{~min}$. The solvent was removed in vacuum, the resulting ammonium salt dissolved in $10 \mathrm{ml}$ of $\mathrm{CH}_{2} \mathrm{Cl}_{2}$ and DIEA $(177 \mu \mathrm{l}$, $131 \mathrm{mg}, 1.01 \mathrm{mmol})$ and $203 \mathrm{mg}(506 \mu \mathrm{mol})$ of 7 were added. After stirring for $20 \mathrm{~h}$ at room temp. $20 \mathrm{~mL}$ of $\mathrm{CH}_{2} \mathrm{Cl}_{2}$ were added and the solution was washed twice with $10 \mathrm{ml}$ of $5 \%$ aqueous $\mathrm{KHSO}_{4}$, once with $10 \mathrm{~mL}$ of $\mathrm{H}_{2} \mathrm{O}$ and twice with $10 \mathrm{~mL}$ of $0.5 \mathrm{~N}$ aqueous $\mathrm{NaHCO}_{3}$. The solution was dried over $\mathrm{MgSO}_{4}$, the solvent evaporated and the crude product subjected 
to column chromatography to give $202 \mathrm{mg}$ (366 $\mu \mathrm{mol}, 72 \%)$ of the Boc-protected tetrapeptide.

This (196 mg, $355 \mathrm{mmol}$ ) was dissolved in $\mathrm{CH}_{2} \mathrm{Cl}_{2}$ containing 33\% TFA (4.5 mL) and stirred at room temperature for $30 \mathrm{~min}$. The solvent was removed in vacuum, the resulting ammonium salt dissolved in $5 \mathrm{~mL}$ of $\mathrm{CH}_{2} \mathrm{Cl}_{2}$ and DIEA (310 $\left.\mu \mathrm{L}, 230 \mathrm{mg}, 1.78 \mathrm{mmol}\right)$ and acetic anhydride $(168 \mu \mathrm{l}, 181 \mathrm{mg}, 1.78 \mathrm{mmol})$ were added. After stirring for $2 \mathrm{~h}, 20 \mathrm{~mL}$ of $\mathrm{CH}_{2} \mathrm{Cl}_{2}$ were added and the solution was washed twice with $10 \mathrm{~mL}$ of $5 \%$ aqueous $\mathrm{KHSO}_{4}$, once with $10 \mathrm{~mL}$ of $\mathrm{H}_{2} \mathrm{O}$ and twice with $10 \mathrm{~mL}$ of $0.5 \mathrm{~N}$ aqueous $\mathrm{NaHCO}_{3}$. The solution was dried over $\mathrm{MgSO}_{4}$, the solvent evaporated and the crude product recrystallised from EtOAc to give $122 \mathrm{mg}(247 \mu \mathrm{mol}, 70 \%)$ of the title compound 10. M.p. $=153-154{ }^{\circ} \mathrm{C} .{ }^{1} \mathrm{H}$ NMR $(600$ $\left.\mathrm{MHz}, \mathrm{CDCl}_{3}\right): \delta=0.92(\mathrm{~d}, J=6.77 \mathrm{~Hz}, 3 \mathrm{H}, \mathrm{COSY}: 5-\mathrm{H}$ or $6-\mathrm{H}), 0.93(\mathrm{~d}, J=6.77 \mathrm{~Hz}, 3 \mathrm{H}$, COSY: 6-H or 5-H), 1.89 - 2.15 (m, 2 H, COSY: 11-H or 12-H), 1.92 (s, 3 H, HMBC: 23-H), 1.99 (s, 3 H, HMBC: 19-H), 2.01 - 2.28 (m, 2 H, COSY: 12-H or 11-H), 2.20 (m, 1 H, COSY: 4-H), 3.71 (s, 3 H, HMBC: HMBC, COSY: 1-H), 3.73 (s, 3 H, HMBC, ROESY: 17-H), 3.77 (dd, $\left.J=17.05 \mathrm{~Hz}, 5.79 \mathrm{~Hz}, 1 \mathrm{H}, \mathrm{COSY}, \mathrm{HMBC}: 8-\mathrm{H}_{\mathrm{a} / \mathrm{b}}\right), 3.77$ - 3.93 (m, $\left.2 \mathrm{H}, \mathrm{COSY}: 13-\mathrm{H}\right)$, $4.03\left(\mathrm{dd}, J=17.05 \mathrm{~Hz}, 6.51 \mathrm{~Hz}, \operatorname{COSY}, \mathrm{HMBC}: 8-\mathrm{H}_{\mathrm{b} / \mathrm{a}}\right), 4.22(\mathrm{dd}, J=15.08 \mathrm{~Hz}, 5.01 \mathrm{~Hz}$, $\left.1 \mathrm{H}, \mathrm{COSY}, \mathrm{HMBC}: 21-\mathrm{H}_{\mathrm{a} / \mathrm{b}}\right), 4.36\left(\mathrm{dd}, J=15.08 \mathrm{~Hz}, 5.01 \mathrm{~Hz}, 1 \mathrm{H}, \mathrm{COSY}, \mathrm{HMBC}: 21-\mathrm{H}_{\mathrm{b} / \mathrm{a}}\right)$, $4.49(\mathrm{dd}, J=8.99 \mathrm{~Hz}, 6.67 \mathrm{~Hz}, 1 \mathrm{H}, \mathrm{COSY}: 3-\mathrm{H}), 4.57(\mathrm{dd}, J=8.33 \mathrm{~Hz}, 4.06 \mathrm{~Hz}, 1 \mathrm{H}$, COSY: 10-H), 6.53 (bm, 1 H, COSY: 21-NH), 7.13 (t, $J=6.03$ Hz, 1 H, COSY: 8-NH), 7.67 (bm, $1 \mathrm{H}$, COSY: 3-NH), 9.92 (s, $1 \mathrm{H}, \mathrm{HMBC}: 15-\mathrm{NH}) .{ }^{13} \mathrm{C} \mathrm{NMR}\left(150 \mathrm{MHz}, \mathrm{CDCl}_{3}\right)$ : $\delta=7.62$ (HMBC: 19-C), 18.22 (HMBC: 5-C or 6-C), 19.06 (HMBC: 6-C or 5-C), 22.94 (HMBC: 23-C), 25.16 (HMBC: 11-C or 12-C), 29.15 (HMBC: 12-C or 11-C), 31.11 (HMBC: 4-C), 34.92 (HMBC: 21-C), 42.87 (HMBC: 8-C), 48.74 (HMBC: 12-C), 52.20 (HMBC: 1-C), 57.53 (HMBC: 3-C), 61.71 (HMBC: 10-C), 62.29 (HMBC: 17-C), 109.59 (HMBC: 18-C), 112.91 (HMBC: 15-C), 128.22 (HMBC: 20-C), 147.03 (HMBC: 16-C), 162.64 (HMBC: 14C), 169.48 (HMBC: 7-C), 170.09 (HMBC: 22-C), 172.83 (HMBC: 9-C), 173.69 (HMBC: 2C). $\mathrm{MS}\left(\mathrm{ESI}, \mathrm{CH}_{2} \mathrm{Cl}_{2} / \mathrm{MeOH}+10 \mathrm{mmol} / \mathrm{L} \mathrm{NH}_{4} \mathrm{Ac}\right): m / z(\%)=494.4$ (100) $\left[\mathrm{MH}^{+}\right], 516.4$ (6) [MNa ${ }^{+}$. Anal. calcd. for $\mathrm{C}_{23} \mathrm{H}_{35} \mathrm{~N}_{5} \mathrm{O}_{7}$ : C, 55.97; H, 7.15; N, 14.19; found C, 55.44; H, 6.82; $\mathrm{N}, 14.01$. 


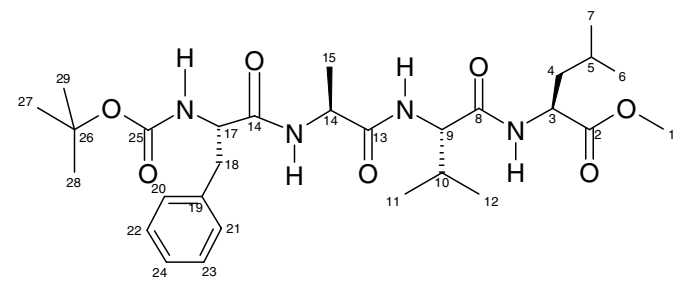

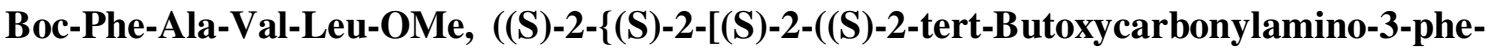
nyl-propionylamino)-propionylamino]-3-methyl-butyrylamino\}-4-methyl-pentanoic acid methyl ester) (13): A solution of Boc-Phe-Ala-OH (336 mg, $1.00 \mathrm{mmol}$ ), HOAt (136 mg, $1.00 \mathrm{mmol})$, HATU (380 mg, $1.00 \mathrm{mmol})$, DIEA (349 $\mu 1,259 \mathrm{mg}$ DIEA, $2.00 \mathrm{mmol})$ and HVal-Leu-OMe $\cdot \mathrm{CF}_{3} \mathrm{COOH}(320 \mathrm{mg}, 1.00 \mathrm{mmol})$ in $5 \mathrm{~mL}$ of dimethylformamide was stirred for $4 \mathrm{~h}$ at room temp. Then the solution was ice cooled and $20 \mathrm{~mL}$ of $\mathrm{H}_{2} \mathrm{O}$ was added. The precipitate was filtered off with suction, dissolved in $100 \mathrm{~mL}$ of $\mathrm{CH}_{2} \mathrm{Cl}_{2}$ washed twice with 20 $\mathrm{mL}$ of $5 \%$ aqueous $\mathrm{KHSO}_{4}$, once with $10 \mathrm{~mL}$ of $\mathrm{H}_{2} \mathrm{O}$ and twice with $20 \mathrm{~mL}$ of $0.5 \mathrm{~N}$ aqueous $\mathrm{NaHCO}_{3}$. The solution was dried over $\mathrm{MgSO}_{4}$ and the solvent evaporated to give $510 \mathrm{mg}$ of the crude product. Recrystallisation from EtOAc/PE gave $449 \mathrm{mg}(797 \mu \mathrm{mol}, 80 \%)$ of compound 13. ${ }^{1} \mathrm{H}$ NMR (600 MHz, DMSO): $\delta=0.87$ (d, $3 \mathrm{H}, J=5.95 \mathrm{~Hz}, \mathrm{COSY}: 6-\mathrm{H}$ or $7-$ H), 0.89 (d, 3 H, $J=6.06 \mathrm{~Hz}$, COSY: 7-H or 6-H), 0.95 (d, 3 H, $J=6.66 \mathrm{~Hz}, \mathrm{COSY}: 11-\mathrm{H}$ or 12-H), $0.97(\mathrm{~d}, 3 \mathrm{H}, J=6.76 \mathrm{~Hz}, \mathrm{COSY}: 12-\mathrm{H}$ or $11-\mathrm{H}), 1.33(\mathrm{~d}, 3 \mathrm{H}, J=6.96 \mathrm{~Hz}$, COSY, HSQC: 15-H), 1.38 (s, 9 H, COSY, HSQC: 27-H bis 29-H), 1.56 (m, $1 \mathrm{H}$, COSY, HSQC: 4- $\left.\mathrm{H}_{\mathrm{a} / \mathrm{b}}\right), 1.63\left(\mathrm{~m}, 1 \mathrm{H}, \mathrm{COSY}:\right.$ 5-H) 1.65 (m, $\left.1 \mathrm{H}, \mathrm{COSY}, \mathrm{HSQC}: 4-\mathrm{H}_{\mathrm{b} / \mathrm{a}}\right), 2.10$ (m, $1 \mathrm{H}$, COSY: 10-H), 2.95 (dd, $1 \mathrm{H}, J=13.92 \mathrm{~Hz}, 7.87 \mathrm{~Hz}$, COSY, HSQC: 18- $\mathrm{H}_{\mathrm{a} / \mathrm{b}}$ ), 3.09 (dd, $1 \mathrm{H}, J=13.93 \mathrm{~Hz}, 5.05 \mathrm{~Hz}, \mathrm{COSY}$, HSQC: 18-H $/ \mathrm{b}$ ), 3.71 (s, $3 \mathrm{H}, \mathrm{HSQC}: 1-\mathrm{H}), 4.46$ (m, $1 \mathrm{H}$, COSY, HSQC: 9-H), 4.61 (m, 1 H, COSY: 3-H), 4.62 (m, 1 H, COSY, HSQC: 17-H), 4.70 (m, $1 \mathrm{H}$, COSY: 14-H), 5.50 (d, $1 \mathrm{H}, J=6.26 \mathrm{~Hz}$, COSY, HMBC: 17-NH), 7.12-7.27 (m, $5 \mathrm{H}, \mathrm{COSY}, \mathrm{HMBC}$ : 20-H bis 24-H), 7.22 (m, $1 \mathrm{H}, \mathrm{COSY}, \mathrm{HMBC}$ : 9-NH), 7.34 (m, $1 \mathrm{H}$, COSY, HMBC: 3-NH), 7.50 (m, $1 \mathrm{H}$, COSY, HMBC: 14-NH). $-{ }^{13} \mathrm{C}$ NMR $(151 \mathrm{MHz}$, DMSO): $\delta=18.44$ (1 C, HSQC: 11-C or 12-C), 18.99 (1 C, HSQC: 12-C or 11-C), 18.99 (1 C, HSQC: 15-C), 21.78 (1 C, HSQC: 6-C or 7-C), 22.81 (1 C, HSQC: 7-C or 6-C), 24.89 (1 C, HSQC: 5-C), 28.30 (3 C, HSQC: 27-C bis 29-C), 31.02 (1 C, HSQC: 10-C), 38.87 (1 C, HSQC: 18-C), 40.81 (1 C, HSQC: 4-C), 48.77 (1 C, HSQC: 14-C), 50.74 (1 C, HSQC: 3-C), 52.19 (1 C, HSQC: 1-C), 55.38 (1 C, HSQC: 17-C), 58.59 (1 C, HSQC: 9-C), 79.87 (1 C, HMBC: 26-C), 126.78 (1 C, HMBC: 24-C), 128.43 (2 C, HSQC: 20-C, 21-C or 22-C, 23-C), 129.40 (2 C, HSQC: 22-C, 23-C or 20-C, 21-C), 138.50 (1 C, HMBC: 19-C), 155.58 (1 C, 
HMBC: 25-C), 171.14 (1 C, HMBC: 8-C), 171.41 (1 C, HMBC: 16-C), 172.47 (1 C, HMBC: 13-C), 173.20 (1 C, HMBC: 2-C). IR (KBr): $\widetilde{v}=3294 \mathrm{~cm}^{-1}$ (s), 3066 (m), 2962 (s), 1748 (sh), $1642(\mathrm{~s}), 1545$ (s).

Boc-D-Pro-Gly-Phe-Ala-Val-Leu-OMe (14): A solution of Boc-Phe-Ala-Val-Leu-OMe (200 mg, $355 \mu \mathrm{mol}$ ) in $9 \mathrm{~mL}$ of $\mathrm{CH}_{2} \mathrm{Cl}_{2}$ containing 33\% TFA was stirred at room temperature for $25 \mathrm{~min}$. The solvent was removed in vacuum, the resulting ammonium salt dissolved in $3 \mathrm{~mL}$ of dimethylformamide, $124 \mu \mathrm{L}(91.7 \mathrm{mg}, 710 \mu \mathrm{mol})$ of DIEA, $137 \mathrm{mg}$ (355 $\mu \mathrm{mol})$ of Boc-D-Pro-Gly-OH, $48.3 \mathrm{mg}(355 \mu \mathrm{mol})$ of HOAt and $135 \mathrm{mg}(355 \mu \mathrm{mol})$ of HATU were added and the solution stirred for $4 \mathrm{~h}$ at room temp. The coupling-product was precipitated by adding $20 \mathrm{~mL}$ of $\mathrm{H}_{2} \mathrm{O}$, filtered off with suction and dissolved in $700 \mathrm{~mL}$ of $\mathrm{CH}_{2} \mathrm{Cl}_{2}$. The solution was washed twice with $150 \mathrm{~mL}$ of $5 \%$ aqueous $\mathrm{KHSO}_{4}$ and twice with $150 \mathrm{~mL}$ of 0.5 $\mathrm{N}$ aqueous $\mathrm{NaHCO}_{3}$. The solution was dried over $\mathrm{MgSO}_{4}$ and the solvent evaporated and recrystallisation from EtOAc/PE $224 \mathrm{mg}$ (307 $\mu \mathrm{mol}, 86 \%$ ) gave hexapeptide 14. ${ }^{1} \mathrm{H}$ NMR (300 MHz, DMSO): $\delta=0.78-0.92$ (m, 12 H), 1.16-1.43 (m, 12 H), 1.40-2.10 (m, 8 H), 2.60-3.14 (m, 2 H), 3.18-3.42 (m, 2 H), 3.42-3.85 (m, 5 H), 3.95-4.62 (m, 5 H), 7.10-7.28 (m, 5 H), 7.60 - 7.77 (m, 1 H), 7.81-8.01 (m, 1 H), 8.01-8.12 (m, 1 H), 8.21-8.37 (m, 2 H).

Boc-MOPAS-D-Pro-Gly-Phe-Ala-Val-Leu-OMe (15): A solution of Boc-D-Pro-Gly-PheAla-Val-Leu-OMe $(223 \mathrm{mg}, 307 \mu \mathrm{mol})$ in $6 \mathrm{~mL}$ of $\mathrm{CH}_{2} \mathrm{Cl}_{2}$ containing 33\% TFA was stirred at room temp. for $25 \mathrm{~min}$. The solvent was removed in vacuum, the resulting ammonium salt dissolved in $3 \mathrm{~mL}$ of dimethylformamide, DIEA $(107 \mu \mathrm{L}, 79.3 \mathrm{mg}, 614 \mu \mathrm{mol})$ and BocMOPAS-OBt (123 mg, $307 \mu \mathrm{mol})$ were added and stirred for $23 \mathrm{~h}$ at room temp. The solution was ice cooled, $20 \mathrm{~mL}$ of $\mathrm{H}_{2} \mathrm{O}$ added, the precipitate filtered off with suction and dissolved in $300 \mathrm{~mL}$ of $\mathrm{CH}_{2} \mathrm{Cl}_{2}$. The solution was washed twice with $50 \mathrm{~mL}$ of $5 \%$ aqueous $\mathrm{KHSO}_{4}$ and twice with $50 \mathrm{~mL}$ of $0.5 \mathrm{~N}$ aqueous $\mathrm{NaHCO}_{3}$, dried over $\mathrm{MgSO}_{4}$ and the solvent evaporated. Recrystallisation of the crude product gave $243 \mathrm{mg}(275 \mu \mathrm{mol}, 90 \%)$ of compound 15. ${ }^{1} \mathrm{H}$ NMR (300 MHz, DMSO): $\delta=0.75-0.92$ (m, 12 H), 1.19 (d, 3 H, J = 7.14 Hz), 1.37 (s, 9 H), 1.40-1.67 (m, 3 H), 1.70-2.10 (m, 7 H), 2.07 - 2.20 (m, 1 H), 2.85 (dd, 1 H, J = 10.29 Hz, $13.31 \mathrm{~Hz}), 3.00(\mathrm{dd}, 1 \mathrm{H}, J=3.57 \mathrm{~Hz}, 13.31 \mathrm{~Hz}), 3.45$ (dd, $1 \mathrm{H}, J=4.94 \mathrm{~Hz}, 17.02 \mathrm{~Hz}), 3.45$ (s, 3 H), 3.50 - 3.69 (m, 1 H), 3.63 (s, 3 H), 3.75 (dd, 1 H, J =6.17, 17.02 Hz), 4.04 (m, 2 H), 4.15-4.40 (m, $3 \mathrm{H}), 4.40-4.50(\mathrm{~m}, 1 \mathrm{H}), 4.50-4.61(\mathrm{~m}, 1 \mathrm{H}), 7.00(\mathrm{t}, 1 \mathrm{H}, J=5.49 \mathrm{~Hz}), 7.12-$ $7.30(\mathrm{~m}, 5 \mathrm{H}), 7.68(\mathrm{~d}, 1 \mathrm{H}, J=8.78 \mathrm{~Hz}), 8.08(\mathrm{~d}, 1 \mathrm{H}, J=8.51 \mathrm{~Hz}), 8.14(\mathrm{~m}, 1 \mathrm{H}), 8.31(\mathrm{~d}$, 
$1 \mathrm{H}, J=7.68 \mathrm{~Hz}), 8.34(\mathrm{~d}, 1 \mathrm{H}, J=7.41 \mathrm{~Hz}), 10.42(\mathrm{~s}, 1 \mathrm{H}) . \mathrm{MS}\left(\mathrm{ESI}, \mathrm{CH}_{2} \mathrm{Cl}_{2} / \mathrm{MeOH}+10\right.$

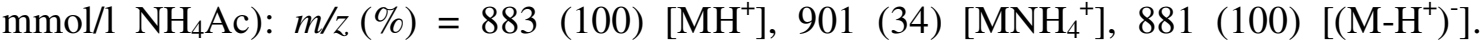
IR (KBr): $\tilde{v}=3429 \mathrm{~cm}^{-1}$ (s), $3298(\mathrm{sh}), 3068(\mathrm{w}), 2963(\mathrm{~m}), 1637$ (s), $1527(\mathrm{~m}), 1457(\mathrm{w})$, $1391(w), 1329(w)$.

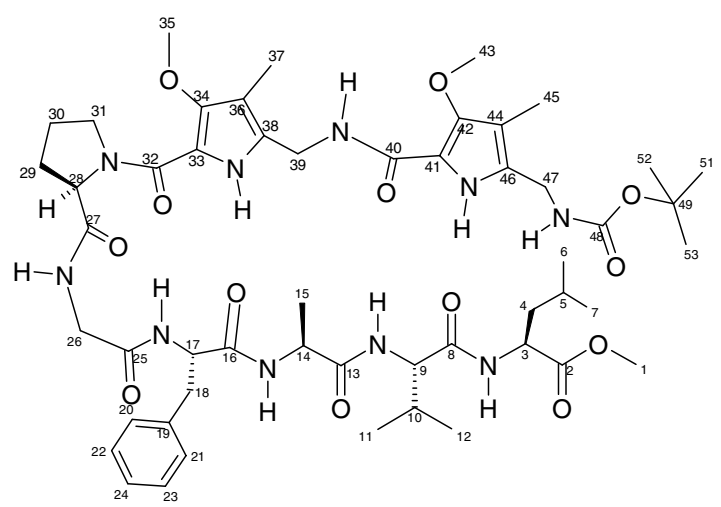

Boc-MOPAS-D-Pro-Gly-Phe-Ala-Val-Leu-OMe (16): A solution of Boc-MOPAS-D-ProGly-Phe-Ala-Val-Leu-OMe (240 mg, $272 \mu \mathrm{mol})$ in $9 \mathrm{~mL}$ of $\mathrm{CH}_{2} \mathrm{Cl}_{2}$ containing 33\% TFA was stirred at room temp. for $25 \mathrm{~min}$. The solvent was removed in vacuum, the resulting ammonium salt dissolved in $3 \mathrm{~mL}$ of dimethylformamide, DIEA $(94.9 \mu 1,70.3 \mathrm{mg}, 544 \mu \mathrm{mol})$ and Boc-MOPAS-OBt (109 mg, $272 \mu \mathrm{mol})$ were added and stirred for $20 \mathrm{~h}$ at room temp. The solution was ice cooled, $20 \mathrm{~mL}$ of $\mathrm{H}_{2} \mathrm{O}$ added, the precipitate filtered off with suction and dissolved in $150 \mathrm{~mL}$ of $\mathrm{CH}_{2} \mathrm{Cl}_{2}$. The solution was washed twice with $30 \mathrm{~mL}$ of $5 \%$ aqueous $\mathrm{KHSO}_{4}$ and twice with $30 \mathrm{~mL}$ of $0.5 \mathrm{~N}$ aqueous $\mathrm{NaHCO}_{3}$, dried over $\mathrm{MgSO}_{4}$ and the solvent evaporated. Column chromatography using 0-5\% EtOH in EtOAc as eluent gave $220 \mathrm{mg}$ (222 $\mu \mathrm{mol}, 77 \%)$ of compound 16. ${ }^{1} \mathrm{H} \mathrm{NMR}\left(600 \mathrm{MHz}, \mathrm{CDCl}_{3}\right): \delta=0.82(\mathrm{~d}, 3 \mathrm{H}, J=6.30 \mathrm{~Hz}$, COSY: 6-H or 7-H), 0.85 (d, 3 H, $J=6.18 \mathrm{~Hz}, \mathrm{COSY}$ : 7-H or 6-H), 0.99 (d, $3 \mathrm{H}, J=6.65$ Hz, COSY: 11-H or 12-H), 1.02 (d, 3 H, $J=6.77 \mathrm{~Hz}$, COSY: 12-H or 11-H), 1.09 (d, $3 \mathrm{H}$, $J=6.65 \mathrm{~Hz}, \mathrm{COSY}$, HSQC: 15-H), 1.38 (s, $9 \mathrm{H}$, HSQC: 50-H bis 52-H), 1.55 (m, $1 \mathrm{H}$, COSY, HSQC: 4-Ha/b), 1.57 (m, $1 \mathrm{H}$, COSY: 5-H), 1.65 (m, $\left.1 \mathrm{H}, \mathrm{COSY}, \mathrm{HSQC}: 4-\mathrm{H}_{\mathrm{b} / \mathrm{a}}\right), 2.03$ (m, 1 H, COSY, HSQC: 30-H $\mathrm{a}_{\mathrm{b}}$ ), 2.07 (m, $1 \mathrm{H}, \mathrm{COSY}$ : 10-H), 2.08 (s, $3 \mathrm{H}, \mathrm{COSY}, \mathrm{HMBC}$ 45-H), 2.09 (m, $1 \mathrm{H}, \mathrm{COSY}, \mathrm{HSQC}: 30-\mathrm{H}_{\mathrm{b} / \mathrm{a}}$ ), 2.14 (s, $3 \mathrm{H}, \mathrm{COSY}, \mathrm{HMBC}$ : 37-H), 2.20 (m, 2 H, COSY, HSQC: 29-H), 3.15 (m, 2 H, COSY, HSQC: 18-H), 3.71 (s, 3 H, HSQC: 1-H), 3.76 (m, 1 H, HSQC, HMBC: 26- $\mathrm{H}_{\mathrm{a} / \mathrm{b}}$ ), 3.77 (m, $1 \mathrm{H}$, HSQC, HMBC: 31- $\left.\mathrm{H}_{\mathrm{a} / \mathrm{b}}\right), 3.80$ (s, $3 \mathrm{H}$, HMBC: 35-H), 3.81 (s, 3 H, HMBC: 43-H), 4.02 (m, 1 H, HSQC, HMBC: 26- $\mathrm{H}_{\mathrm{b} / \mathrm{a}}$ ), 4.04 (m, $\left.1 \mathrm{H}, \mathrm{HSQC}, \mathrm{HMBC}: 31-\mathrm{H}_{\mathrm{b} / \mathrm{a}}\right), 4.15$ (m, $\left.1 \mathrm{H}, \mathrm{HMBC}, \mathrm{HSQC}: 47-\mathrm{H}_{\mathrm{a} / \mathrm{b}}\right), 4.17$ (m, $1 \mathrm{H}, \mathrm{HMBC}$, 
HSQC: 47- $\left.\mathrm{H}_{\mathrm{a} / \mathrm{b}}\right), 4.51(\mathrm{~m}, 1 \mathrm{H}, \mathrm{COSY}: 3-\mathrm{H}), 4.61(\mathrm{dd}, 1 \mathrm{H}, J=8.14 \mathrm{~Hz}, 2.58 \mathrm{~Hz}, \mathrm{COSY}: 28$ H), 4.66 (m, 2 H, HMBC, HSQC: 39-H), 4.76 (t, 1 H, $J=8.44$ Hz, COSY, HSQC: 9-H), 5.00 (t, $1 \mathrm{H}, J=6.08 \mathrm{~Hz}, \mathrm{COSY}$, HSQC: 14-H), 5.34 (m, $1 \mathrm{H}, \mathrm{COSY}: 17-\mathrm{H}), 5.73$ (t, $1 \mathrm{H}, J=6.02$ Hz, COSY, HMBC: 47-NH), 6.49 (m, 1 H, COSY, HMBC: 26-NH), 7.10 (m, 1 H, COSY, HMBC: 39-NH), 7.09 (m, 1 H, COSY, HMBC: 24-H), 7.22 (m, 2 H, COSY, HMBC: 20-H bis $23-\mathrm{H}), 7.26$ (m, $2 \mathrm{H}$, COSY, HMBC: $20-\mathrm{H}$ bis $23-\mathrm{H}), 8.18(\mathrm{~d}, 1 \mathrm{H}, J=8.17 \mathrm{~Hz}, \mathrm{COSY}$, HMBC: 17-NH), 8.66 (m, 1 H, COSY, HMBC: 3-NH), 8.80 (m, 1 H, COSY, HMBC: 14NH), 9.05 (d, 1 H, J = 7.04 Hz, COSY, HMBC: 9-NH), 11.01 (bs, 1 H, COSY, HMBC: 41NH), 11.39 (bs, 1 H, COSY, HMBC: 33-NH).

${ }^{13} \mathrm{C}$ NMR (151 MHz, $\left.\mathrm{CDCl}_{3}\right): \delta=7.65$ (1 C, HSQC: 37-C), 8.11 (1 C, 45-C), 18.83 (1 C, HSQC: 11-C or 12-C), 19.23 (1 C, HSQC: 12-C or 11-C), 20.05 (1 C, HSQC: 15-C), 22.06 (1 C, HSQC: 6-C or 7-C), 22.66 (1 C, HSQC: 7-C or 6-C), 24.92 (1 C, HSQC: 5-C), 25.19 (1 C, HSQC: 30-C), 28.40 (3 C, HSQC: 50-C bis 52-C), 28.80 (1 C, HSQC: 29-C), 31.70 (1 C, HSQC: 10-C), 34.66 (1 C, HSQC: 39-C), 35.27 (1 C, HSQC: 47-C), 39.74 (1 C, HSQC: 18-C), 40.16 (1 C, HSQC: 4-C), 42.70 (1 C, HSQC: 26-C), 48.33 (1 C, HSQC: 31-C), 48.54 (1 C, HSQC: 14-C), 51.17 (1 C, HSQC: 3-C), 52.10 (1 C, HSQC: 1-C), 53.89 (1 C, HSQC: 17-C), 58.08 (1 C, HSQC: 9-C), 61.29 (1 C, HSQC: 43-C), 61.83 (1 C, HSQC: 28-C), 62.58 (1 C, HSQC: 35-C), 78.95 (1 C, HSQC: 49-C), 108.00 (1 C, HMBC: 44-C), 109.28 (1 C, HMBC: 36-C), 112.01 (1 C, HMBC: 41-C), 112.76 (1 C, HMBC: 33-C), 126.59 (1 C, HMBC: 24-C), 127.96 (1 C, HMBC: C-38), 128.16 (2 C, HSQC: 20-C, 21-C or 22-C, 23-C), 129.56 (1 C, HMBC: 46-C), 129.58 (2 C, HSQC: 22-C, 23-C or 20-C, 21-C), 136.86 (1 C, HMBC: 19-C), 147.06 (1 C, HMBC: 34-C), 147.26 (1 C, HMBC: 42-C), 155.68 (1 C, HMBC: 48-C),160.89 (1 C, HMBC: 40-C), 163.13 (1 C, HMBC: 32-C), 168.70 (1 C, HMBC: 25-C), 171.23 (1 C, HMBC: 16-C), 172.26 (1 C, HMBC: 27-C), 172.54 (1 C, HMBC: 13-C), 172.79 (1 C, HMBC: 2-C), 173.27 (1 C, HMBC: 8-C).

${ }^{1} \mathrm{H}$ NMR (600 MHz, DMSO): $\delta(\mathrm{ppm})=0.81(\mathrm{~d}, 3 \mathrm{H}, J=6.48 \mathrm{~Hz}, \mathrm{COSY}: 6-\mathrm{H}$ or 7-H), 0.87 (d, $3 \mathrm{H}, J=6.54 \mathrm{~Hz}, \mathrm{COSY}: 7-\mathrm{H}$ or $6-\mathrm{H}), 0.85(\mathrm{~d}, 3 \mathrm{H}, J=6.65 \mathrm{~Hz}, \mathrm{COSY}: 11-\mathrm{H}$ or 12-H), $0.85(\mathrm{~d}, 3 \mathrm{H}, J=6.71 \mathrm{~Hz}, \mathrm{COSY}: 12-\mathrm{H}$ or $11-\mathrm{H}), 1.15(\mathrm{~d}, J=7.10 \mathrm{~Hz}, 3 \mathrm{H}, \mathrm{COSY}$, HSQC: 15-H), 1.36 (s, 9 H, HSQC: 50-H bis 52-H), 1.48 (m, 1 H, COSY, HSQC: 4- $\left.\mathrm{H}_{\mathrm{a} / \mathrm{b}}\right), 1.55$ (m, $1 \mathrm{H}, \mathrm{COSY}: 5-\mathrm{H}), 1.61\left(\mathrm{~m}, 1 \mathrm{H}, \mathrm{COSY}\right.$, HSQC: 4-H $\left.\mathrm{b}_{\mathrm{a}}\right), 1.77(\mathrm{~m}, 1 \mathrm{H}, \mathrm{COSY}, \mathrm{HSQC}: 30-$

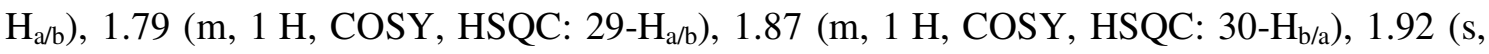
3 H, COSY, HMBC: 45-H), 1.96 (m, 1 H, COSY: 10-H), 1.98 (s, 3 H, COSY, HMBC: 37-H), $2.12(\mathrm{~m}, 1 \mathrm{H}, \mathrm{COSY}$, HSQC: 29-H $\mathrm{b} / \mathrm{a}), 2.86(\mathrm{dd}, 1 \mathrm{H}, J=10.26 \mathrm{~Hz}, 13.50 \mathrm{~Hz}, \mathrm{COSY}, \mathrm{HSQC}$ : $\left.18-\mathrm{H}_{\mathrm{a} / \mathrm{b}}\right), 3.00\left(\mathrm{dd}, 1 \mathrm{H}, J=3.70 \mathrm{~Hz}, 13.50 \mathrm{~Hz}\right.$, COSY, HSQC: 18- $\left.\mathrm{H}_{\mathrm{b} / \mathrm{a}}\right), 3.43(\mathrm{dd}, 1 \mathrm{H}$, 
$J=16.85 \mathrm{~Hz}, 5.18 \mathrm{~Hz}, \mathrm{HSQC}$, HMBC: 26- $\mathrm{H}_{\mathrm{a} / \mathrm{b}}$ ), 3.60 (s, $\left.3 \mathrm{H}, \mathrm{HSQC}: 1-\mathrm{H}\right), 3.64$ (s, $3 \mathrm{H}$, HMBC: 35-H), 3.66 (m, 2 H, HSQC, HMBC: 31-H), 3.70 (s, 3 H, HMBC: 43-H), 3.76 (dd, $1 \mathrm{H}, J=16.85 \mathrm{~Hz}, 6.46 \mathrm{~Hz}$, HSQC, HMBC: 26-H $\mathrm{b} / \mathrm{a}), 4.01$ (m, $2 \mathrm{H}, \mathrm{HMBC}, \mathrm{HSQC}: 47-\mathrm{H})$, 4.21 (dd, $1 \mathrm{H}, J=8.94 \mathrm{~Hz}, 6.92 \mathrm{~Hz}$, COSY, HSQC: 9-H), 4.51 (ddd1 H, , $=10.04 \mathrm{~Hz}, 7.46$ Hz, 5.05 Hz, COSY: 3-H), 4.33 (m, 2 H, HMBC, HSQC: 39-H), 4.39 (m, 1 H, COSY, HSQC: 14-H), 4.46 (m, 1 H, COSY: 28-H), 4.57 (ddd, $1 \mathrm{H}, J=9.92 \mathrm{~Hz}, 8.72 \mathrm{~Hz}, 3.73 \mathrm{~Hz}, \mathrm{COSY}$ : 17-H), 6.95 (t, $1 \mathrm{H}, J=5.60 \mathrm{~Hz}, \mathrm{COSY}, \mathrm{HMBC}: 47-\mathrm{NH}), 7.17$ (m, $1 \mathrm{H}, \mathrm{COSY}, \mathrm{HMBC}$ : 24H), 7.23 (m, 2 H, COSY, HMBC: 20-H bis 23-H), 7.26 (m, 2 H, COSY, HMBC: 20-H bis 23H), 7.36 (t, $1 \mathrm{H}, J=5.94 \mathrm{~Hz}, \mathrm{COSY}, \mathrm{HMBC}: 39-\mathrm{NH}), 7.70$ (d, $1 \mathrm{H}, J=8.94 \mathrm{~Hz}, \mathrm{COSY}$, HMBC: 9-NH), 8.06 (d, $1 \mathrm{H}, J=8.50 \mathrm{~Hz}, \mathrm{COSY}, \mathrm{HMBC}: 17-\mathrm{NH}), 8.12$ (m, $1 \mathrm{H}, \mathrm{COSY}$, HMBC: 26-NH), 8.30 (d, $1 \mathrm{H}, J=7.46 \mathrm{~Hz}, \mathrm{COSY}, \mathrm{HMBC}: 3-\mathrm{NH}), 8.34$ (d, $1 \mathrm{H}, J=7.52 \mathrm{~Hz}$, COSY, HMBC: 14-NH), 10.55 (bs, 1 H, COSY, HMBC: 41-NH), 10.70 (bs, $1 \mathrm{H}, \mathrm{COSY}$, HMBC: 33-NH).

${ }^{13} \mathrm{C}$ NMR (151 MHz, DMSO): $\delta=7.36$ (1 C, HSQC: 37-C), 7.54 (1 C, HSQC: 45-C), 17.77 (1 C, HSQC: 15-C), 17.89 (1 C, HSQC: 11-C or 12-C), 18.92 (1 C, HSQC: 12-C or 11-C), 21.08 (1 C, HSQC: 6-C or 7-C), 22.59 (1 C, HSQC: 7-C or 6-C), 24.10 (1 C, HSQC: 5-C), 24.38 (1 C, HSQC: 30-C), 28.10 (3 C, HSQC: 50-C bis 52-C), 29.00 (1 C, HSQC: 29-C), 30.75 (1 C, HSQC: 10-C), 33.28 (1 C, HSQC: 39-C), 35.06 (1 C, HSQC: 47-C), 37.36 (1 C, HSQC: 18-C), 39.43 (1 C, HSQC: 4-C), 41.91 (1 C, HSQC: 26-C), 48.01 (1 C, HSQC: 31-C), 48.24 (1 C, HSQC: 14-C), 50.08 (1 C, HSQC: 3-C), 51.65 (1 C, HSQC: 1-C), 53.67 (1 C, HSQC: 17-C), 57.09 (1 C, HSQC: 9-C), 61.09 (1 C, HSQC: 43-C), 60.36 (1 C, HSQC: 28-C), 61.55 (1 C, HSQC: 35-C), 77.77 (1 C, HSQC: 49-C), 106.95 (1 C, HMBC: 44-C), 107.42 (1 C, HMBC: 36-C), 111.59 (1 C, HMBC: 41-C), 112.44 (1 C, HMBC: 33-C), 126.08 (1 C, HMBC: 24-C), 128.10 (1 C, HMBC: 38), 127.89 (2 C, HSQC: 20-C, 21-C or 22-C, 23-C), 128.30 (1 C, HMBC: 46-C), 129.20 (2 C, HSQC: 22-C, 23-C or 20-C, 21-C), 137.74 (1 C, HMBC: 19-C), 145.79 (1 C, HMBC: 34-C), 146.34 (1 C, HMBC: 42-C), 155.26 (1 C, HMBC: 48-C), 159.58 (1 C, HMBC: 40-C), 161.39 (1 C, HMBC: 32-C), 168.63 (1 C, HMBC: 25-C), 170.97 (1 C, HMBC: 8-C), 171.14 (1 C, HMBC: 16-C), 172.21 (1 C, HMBC: 27-C), 171.67 (1 C, HMBC: 13-C), 172.59 (1 C, HMBC: 2-C).- MS (ESI, $\mathrm{CH}_{2} \mathrm{Cl}_{2} / \mathrm{MeOH}+$ $\left.10 \mathrm{mmol} / \mathrm{l} \mathrm{NH} \mathrm{N}_{4} \mathrm{Ac}\right): m / z(\%)=1050(100)\left[\mathrm{MH}^{+}\right], 1067$ (33) $\left[\mathrm{MNH}_{4}{ }^{+}\right], 1073$ (32) $\left[\mathrm{MNa}^{+}\right]$,

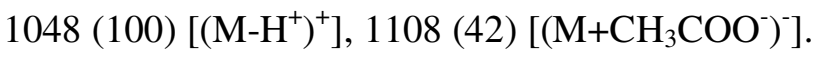

IR (KBr): $\tilde{v}=3420 \mathrm{~cm}^{-1}$ (s), 3298 (sh), 3071 (w), 2962 (m), 1645 (s), 1535 (m), 1456 (w), 1391 (w), 1367 (w). Anal. calcd. for $\mathrm{C}_{52} \mathrm{H}_{76} \mathrm{~N}_{10} \mathrm{O}_{13}$ : C, 59.51; H, 7.30; N, 13.35; found C, 59.02; H, 7.28; N, 12.87 . 


\section{Boc-(MOPAS $)_{2}$-OEt (Ethyl 5-(\{[5-(tert-butoxycarbonylamino-methyl)-3-methoxy-4- methyl-1H-pyrrole-2-carbonyl]-amino\}-methyl)-3-methoxy-4-methyl-1H-pyrrole-2-}

carboxylate) (18): A solution of Boc-MOPAS-OEt (5) $(198 \mathrm{mg}, 635 \mu \mathrm{mol})$ in $4.5 \mathrm{~mL}$ of $\mathrm{CH}_{2} \mathrm{Cl}_{2}$ containing $33 \%$ of TFA was stirred at room temperature for $25 \mathrm{~min}$. The solvent was removed in vacuum, the resulting ammonium salt was dissolved in $7 \mathrm{~mL}$ of $\mathrm{CH}_{2} \mathrm{Cl}_{2}$ and 333 $\mu \mathrm{l}(246 \mathrm{mg}, 1.90 \mathrm{mmol})$ of DIEA and $255 \mathrm{mg}(635 \mu \mathrm{mol})$ of 7 were added After stirring for $15 \mathrm{~h}$ at room temp. $25 \mathrm{~mL}$ of $\mathrm{CH}_{2} \mathrm{Cl}_{2}$ were added and the solution was washed twice with 10 $\mathrm{mL}$ of $5 \% \mathrm{KHSO}_{4}$ aq., once with $10 \mathrm{~mL}$ of $\mathrm{H}_{2} \mathrm{O}$ and twice with $10 \mathrm{~mL}$ of $0.5 \mathrm{~N} \mathrm{NaHCO}_{3}$. The solution was dried over $\mathrm{MgSO}_{4}$, the solvent was evaporated to give $293 \mathrm{mg}$ of crude 17, which was purified by column chromatography $\left(\mathrm{CHCl}_{3} / \mathrm{EtOAc} / \mathrm{EtOH} 72: 18: 10\right)$ to yield $272 \mathrm{mg}(89 \%)$ of 17. $R_{\mathrm{f}}=0.36$. M.p. $=162-164.5{ }^{\circ} \mathrm{C} .{ }^{1} \mathrm{H}$ NMR $\left(300 \mathrm{MHz}, \mathrm{CDCl}_{3}\right): \delta=1.37$ (t, J=7.17 Hz, $3 \mathrm{H}), 1.43$ (s, $9 \mathrm{H}), 1.60(\mathrm{~s}, 3 \mathrm{H}), 2.03$ (s, $3 \mathrm{H}), 3.78$ (s, $3 \mathrm{H}), 3.86(\mathrm{~s}, 3 \mathrm{H})$, $4.21(\mathrm{~d}, J=6.21 \mathrm{~Hz}, 2 \mathrm{H}), 4.33(\mathrm{q}, J=7.17 \mathrm{~Hz}, 2 \mathrm{H}), 4.57$ (d, $J=5.62 \mathrm{~Hz}, 2 \mathrm{H}), 5.22(\mathrm{bm}$, $1 \mathrm{H}), 7.15(\mathrm{t}, J=5.62 \mathrm{~Hz}, 1 \mathrm{H}), 9.82(\mathrm{~s}, 1 \mathrm{H}), 10.08(\mathrm{~s}, 1 \mathrm{H}) .-{ }^{13} \mathrm{C} \mathrm{NMR}\left(75 \mathrm{MHz}, \mathrm{CDCl}_{3}\right): \delta$ = 7.26 (+, 1 C), 8.09 (+, 1 C), 14.40 (+, 1 C), 28.38 (+, 3 C), 34.57 (-, 1 C), 35.59 (-, $1 \mathrm{C})$, $60.30(-, 1 \mathrm{C}), 61.27$ (+, $1 \mathrm{C}), 62.26$ (+, $1 \mathrm{C}), 108.15\left(\mathrm{C}_{\text {quat }}, 1 \mathrm{C}\right), 110.02\left(\mathrm{C}_{\text {quat }}, 1 \mathrm{C}\right), 110.40$ $\left(\mathrm{C}_{\text {quat }}, 1 \mathrm{C}\right), 112.40\left(\mathrm{C}_{\text {quat }}, 1 \mathrm{C}\right), 128.70\left(\mathrm{C}_{\text {quat }}, 1 \mathrm{C}\right), 130.01\left(\mathrm{C}_{\text {quat }}, 1 \mathrm{C}\right), 147.34\left(\mathrm{C}_{\text {quat }}, 1 \mathrm{C}\right)$, $151.36\left(\mathrm{C}_{\text {quat }}, 1 \mathrm{C}\right), 155.74\left(\mathrm{C}_{\text {quat }}, 1 \mathrm{C}\right), 161.21\left(\mathrm{C}_{\text {quat }}, 1 \mathrm{C}\right), 161.54\left(\mathrm{C}_{\text {quat }}, 1 \mathrm{C}\right) . \mathrm{MS}(\mathrm{ESI}$, $\left.\mathrm{CH}_{2} \mathrm{Cl}_{2} / \mathrm{MeOH}+10 \mathrm{mmol} / \mathrm{l} \mathrm{NH} \mathrm{NH}_{4} \mathrm{Ac}\right): m / z(\%)=477.2(100)\left[\left(\mathrm{M}-\mathrm{H}^{+}\right)^{-}\right]$. Anal. calcd. for $\mathrm{C}_{23} \mathrm{H}_{34} \mathrm{~N}_{4} \mathrm{O}_{7}$ : C, 57.73; H, 7.16; N, 11.71; found C, 57.50; H, 6.73; N, 11.69. 


\section{X-ray crystal structures}

\section{3-Hydroxy-4-methyl-1H-pyrrole-2-carboxylic acid ethyl ester, 1.}

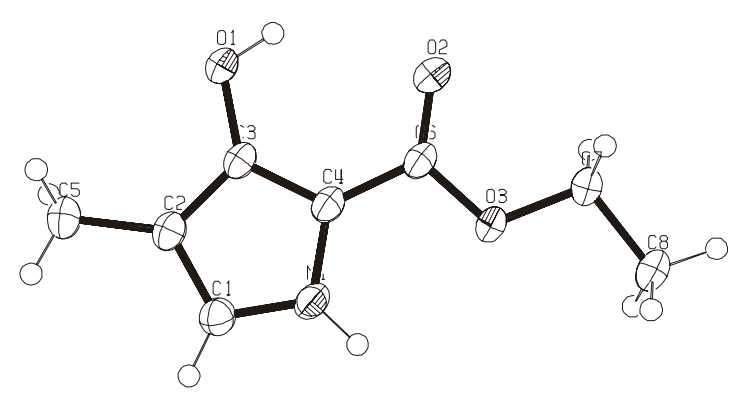

Table 1. Crystal data and structure refinement for compound 1

\begin{tabular}{|c|c|}
\hline Crystal Data & \\
\hline Empirical formula & $\mathrm{C} 8 \mathrm{H} 11 \mathrm{~N} \mathrm{O} 3$ \\
\hline Formula weight & 169.18 \\
\hline Crystal size & $0.55 \times 0.55 \times 0.50 \mathrm{~mm}$ \\
\hline Crystal description & prism \\
\hline Crystal colour & translucent, colourless with a touch of brown \\
\hline Crystal system & Monoclinic \\
\hline Space group & $\mathrm{P} 21 / \mathrm{n}$ \\
\hline \multirow[t]{3}{*}{ Unit cell dimensions } & $\mathrm{a}=7.0752(6) \mathrm{A}$ alpha $=90 \mathrm{deg}$. \\
\hline & $\mathrm{b}=11.9826(12) \mathrm{A} \quad$ beta $=93.814(10) \mathrm{deg}$. \\
\hline & $\mathrm{c}=9.9718(8) \mathrm{A} \quad$ gamma $=90 \mathrm{deg}$ \\
\hline Volume & $843.53(13) \mathrm{A}^{\wedge} 3$ \\
\hline Z, Calculated density & $4,1.332 \mathrm{Mg} / \mathrm{m}^{\wedge} 3$ \\
\hline Absorption coefficient & $0.102 \mathrm{~mm}^{\wedge}-1$ \\
\hline $\mathrm{F}(000)$ & 360 \\
\hline \multicolumn{2}{|l|}{ Data Collection } \\
\hline Measurement device type & STOE-IPDS diffractometer \\
\hline Measuremnet method & rotation \\
\hline Temperature & $173(1) \mathrm{K}$ \\
\hline Wavelength & $0.71073 \mathrm{~A}$ \\
\hline Monochromator & graphite \\
\hline Theta range for data collection & 2.66 to $27.87 \mathrm{deg}$. \\
\hline
\end{tabular}




\begin{tabular}{c|c} 
Index ranges & $-9<=\mathrm{h}<=9,-15<=\mathrm{k}<=15,-12<=\mathrm{l}<=12$ \\
\hline Reflections collected / unique & $13385 / 1872[\mathrm{R}(\mathrm{int})=0.0414]$ \\
\hline Reflections greater I $>2 \backslash \mathrm{ls}(\mathrm{I})$ & 1674 \\
\hline Absorption correction & None \\
\hline Refinement & Full-matrix least-squares on $\mathrm{F}^{\wedge} 2$ \\
\hline Refinement method & $1872 / 0 / 124$ \\
\hline Hydrogen treatment & 1.062 \\
\hline Data / restraints / parameters & $\mathrm{R} 1=0.0378, \mathrm{wR} 2=0.1023$ \\
\hline Goodness-of-fit on F^2 & $\mathrm{R} 1=0.0418, \mathrm{wR} 2=0.1053$ \\
\hline Final R indices [I $>2$ sigma(I)] & $?$ \\
\hline R indices (all data) & 0.280 and $-0.152 \mathrm{e} . \mathrm{A}^{\wedge}-3$
\end{tabular}

Table 2. Atomic coordinates ( $\times 10^{\wedge} 4$ ) and equivalent isotropic displacement parameters $\left(\mathrm{A}^{\wedge} 2 \times 10^{\wedge} 3\right)$ for compound $\mathbf{1}$. U(eq) is defined as one third of the trace of the orthogonalized Uij tensor.

\begin{tabular}{c|c|c|c|c} 
& $\mathrm{x}$ & $\mathrm{y}$ & $\mathrm{z}$ & $\mathrm{U}(\mathrm{eq})$ \\
\hline & & & & \\
\hline $\mathrm{O}(1)$ & $2657(1)$ & $3559(1)$ & $9863(1)$ & $31(1)$ \\
\hline $\mathrm{O}(2)$ & $-1101(1)$ & $4464(1)$ & $8887(1)$ & $38(1)$ \\
\hline $\mathrm{O}(3)$ & $-2502(1)$ & $3520(1)$ & $7112(1)$ & $30(1)$ \\
\hline $\mathrm{N}(1)$ & $462(1)$ & $2031(1)$ & $7112(1)$ & $29(1)$ \\
\hline $\mathrm{C}(1)$ & $2097(2)$ & $1453(1)$ & $7359(1)$ & $30(1)$ \\
\hline $\mathrm{C}(2)$ & $3160(2)$ & $1928(1)$ & $8435(1)$ & $28(1)$ \\
\hline $\mathrm{C}(3)$ & $2092(1)$ & $2851(1)$ & $8838(1)$ & $25(1)$ \\
\hline $\mathrm{C}(4)$ & $422(1)$ & $2910(1)$ & $8012(1)$ & $26(1)$ \\
\hline $\mathrm{C}(5)$ & $5054(2)$ & $1537(1)$ & $9021(1)$ & $36(1)$ \\
\hline $\mathrm{C}(6)$ & $-1099(2)$ & $3706(1)$ & $8069(1)$ & $26(1)$ \\
\hline $\mathrm{C}(7)$ & $-4132(2)$ & $4263(1)$ & $7117(1)$ & $29(1)$ \\
\hline $\mathrm{C}(8)$ & $-5527(2)$ & $3872(1)$ & $5997(1)$ & $31(1)$ \\
& & & &
\end{tabular}

Table 3. Bond lengths $[\mathrm{A}]$ and angles [deg] for compound $\mathbf{1}$

\begin{tabular}{c|c}
$\mathrm{O}(1)-\mathrm{C}(3)$ & $1.3673(14)$ \\
\hline $\mathrm{O}(2)-\mathrm{C}(6)$ & $1.2212(14)$ \\
\hline $\mathrm{O}(3)-\mathrm{C}(6)$ & $1.3482(13)$ \\
\hline $\mathrm{O}(3)-\mathrm{C}(7)$ & $1.4567(13)$
\end{tabular}




\begin{tabular}{|c|c|}
\hline $\mathrm{O}(1)-\mathrm{H}(1 \mathrm{O})$ & $0.855(16)$ \\
\hline $\mathrm{N}(1)-\mathrm{C}(1)$ & $1.3571(15)$ \\
\hline $\mathrm{N}(1)-\mathrm{C}(4)$ & $1.3847(15)$ \\
\hline $\mathrm{N}(1)-\mathrm{H}(1 \mathrm{~N})$ & $0.891(15)$ \\
\hline $\mathrm{C}(1)-\mathrm{C}(2)$ & $1.3900(16)$ \\
\hline$C(2)-C(5)$ & $1.5013(17)$ \\
\hline $\mathrm{C}(2)-\mathrm{C}(3)$ & $1.4130(15)$ \\
\hline $\mathrm{C}(3)-\mathrm{C}(4)$ & $1.3963(15)$ \\
\hline $\mathrm{C}(4)-\mathrm{C}(6)$ & $1.4420(15)$ \\
\hline $\mathrm{C}(7)-\mathrm{C}(8)$ & $1.5142(16)$ \\
\hline $\mathrm{C}(1)-\mathrm{H}(1)$ & 0.9301 \\
\hline $\mathrm{C}(5)-\mathrm{H}(5 \mathrm{~A})$ & $0.988(17)$ \\
\hline $\mathrm{C}(5)-\mathrm{H}(5 \mathrm{~B})$ & $0.957(16)$ \\
\hline $\mathrm{C}(5)-\mathrm{H}(5 \mathrm{C})$ & $0.929(16)$ \\
\hline $\mathrm{C}(7)-\mathrm{H}(7 \mathrm{~A})$ & 0.9698 \\
\hline $\mathrm{C}(7)-\mathrm{H}(7 \mathrm{~B})$ & 0.9703 \\
\hline $\mathrm{C}(8)-\mathrm{H}(8 \mathrm{~A})$ & 0.9600 \\
\hline $\mathrm{C}(8)-\mathrm{H}(8 \mathrm{~B})$ & 0.9602 \\
\hline $\mathrm{C}(8)-\mathrm{H}(8 \mathrm{C})$ & 0.9601 \\
\hline $\mathrm{C}(6)-\mathrm{O}(3)-\mathrm{C}(7)$ & $116.30(9)$ \\
\hline $\mathrm{C}(3)-\mathrm{O}(1)-\mathrm{H}(1 \mathrm{O})$ & $112.0(10)$ \\
\hline $\mathrm{C}(1)-\mathrm{N}(1)-\mathrm{C}(4)$ & $108.93(9)$ \\
\hline $\mathrm{C}(4)-\mathrm{N}(1)-\mathrm{H}(1 \mathrm{~N})$ & $124.5(10)$ \\
\hline $\mathrm{C}(1)-\mathrm{N}(1)-\mathrm{H}(1 \mathrm{~N})$ & $126.6(10)$ \\
\hline $\mathrm{N}(1)-\mathrm{C}(1)-\mathrm{C}(2)$ & $109.97(10)$ \\
\hline$C(3)-C(2)-C(5)$ & $127.89(11)$ \\
\hline$C(1)-C(2)-C(5)$ & $126.54(10)$ \\
\hline$C(1)-C(2)-C(3)$ & $105.57(9)$ \\
\hline $\mathrm{O}(1)-\mathrm{C}(3)-\mathrm{C}(2)$ & $124.27(9)$ \\
\hline $\mathrm{O}(1)-\mathrm{C}(3)-\mathrm{C}(4)$ & $127.14(10)$ \\
\hline$C(2)-C(3)-C(4)$ & $108.58(10)$ \\
\hline$C(3)-C(4)-C(6)$ & $127.91(10)$ \\
\hline $\mathrm{N}(1)-\mathrm{C}(4)-\mathrm{C}(3)$ & $106.93(9)$ \\
\hline $\mathrm{N}(1)-\mathrm{C}(4)-\mathrm{C}(6)$ & $125.15(10)$ \\
\hline $\mathrm{O}(2)-\mathrm{C}(6)-\mathrm{O}(3)$ & $124.16(10)$ \\
\hline $\mathrm{O}(2)-\mathrm{C}(6)-\mathrm{C}(4)$ & $123.53(10)$ \\
\hline $\mathrm{O}(3)-\mathrm{C}(6)-\mathrm{C}(4)$ & $112.30(9)$ \\
\hline $\mathrm{O}(3)-\mathrm{C}(7)-\mathrm{C}(8)$ & $106.66(9)$ \\
\hline $\mathrm{N}(1)-\mathrm{C}(1)-\mathrm{H}(1)$ & 125.01 \\
\hline
\end{tabular}




\begin{tabular}{c|c}
$\mathrm{C}(2)-\mathrm{C}(1)-\mathrm{H}(1)$ & 125.01 \\
\hline $\mathrm{C}(2)-\mathrm{C}(5)-\mathrm{H}(5 \mathrm{~A})$ & $112.4(8)$ \\
\hline $\mathrm{C}(2)-\mathrm{C}(5)-\mathrm{H}(5 \mathrm{~B})$ & $114.2(9)$ \\
\hline $\mathrm{C}(2)-\mathrm{C}(5)-\mathrm{H}(5 \mathrm{C})$ & $111.1(10)$ \\
\hline $\mathrm{H}(5 \mathrm{~A})-\mathrm{C}(5)-\mathrm{H}(5 \mathrm{~B})$ & $101.4(13)$ \\
\hline $\mathrm{H}(5 \mathrm{~A})-\mathrm{C}(5)-\mathrm{H}(5 \mathrm{C})$ & $110.7(14)$ \\
\hline $\mathrm{H}(5 \mathrm{~B})-\mathrm{C}(5)-\mathrm{H}(5 \mathrm{C})$ & $106.6(13)$ \\
\hline $\mathrm{O}(3)-\mathrm{C}(7)-\mathrm{H}(7 \mathrm{~A})$ & 110.38 \\
\hline $\mathrm{O}(3)-\mathrm{C}(7)-\mathrm{H}(7 \mathrm{~B})$ & 110.38 \\
\hline $\mathrm{C}(8)-\mathrm{C}(7)-\mathrm{H}(7 \mathrm{~A})$ & 110.39 \\
\hline $\mathrm{C}(8)-\mathrm{C}(7)-\mathrm{H}(7 \mathrm{~B})$ & 110.41 \\
\hline $\mathrm{H}(7 \mathrm{~A})-\mathrm{C}(7)-\mathrm{H}(7 \mathrm{~B})$ & 108.62 \\
\hline $\mathrm{C}(7)-\mathrm{C}(8)-\mathrm{H}(8 \mathrm{~A})$ & 109.47 \\
\hline $\mathrm{C}(7)-\mathrm{C}(8)-\mathrm{H}(8 \mathrm{~B})$ & 109.49 \\
\hline $\mathrm{C}(7)-\mathrm{C}(8)-\mathrm{H}(8 \mathrm{C})$ & 109.47 \\
\hline $\mathrm{H}(8 \mathrm{~A})-\mathrm{C}(8)-\mathrm{H}(8 \mathrm{~B})$ & 109.47 \\
\hline $\mathrm{H}(8 \mathrm{~A})-\mathrm{C}(8)-\mathrm{H}(8 \mathrm{C})$ & 109.49 \\
\hline $\mathrm{H}(8 \mathrm{~B})-\mathrm{C}(8)-\mathrm{H}(8 \mathrm{C})$ & 109.44 \\
\hline
\end{tabular}

Table 4. Anisotropic displacement parameters $\left(\mathrm{A}^{\wedge} 2 \times 10^{\wedge} 3\right)$ for compound $\mathbf{1}$. The anisotropic displacement factor exponent takes the form: $-2 \mathrm{pi}^{\wedge} 2\left[\mathrm{~h}^{\wedge} 2 \mathrm{a}^{* \wedge} 2 \mathrm{U} 11+\ldots+2 \mathrm{~h} \mathrm{k} \mathrm{a*} \mathrm{b}^{*} \mathrm{U} 12\right.$ ]

\begin{tabular}{l|l|l|l|l|l|l} 
& $\mathrm{U} 11$ & $\mathrm{U} 22$ & $\mathrm{U} 33$ & $\mathrm{U} 23$ & $\mathrm{U} 13$ & $\mathrm{U} 12$ \\
\hline $\mathrm{O}(1)$ & $28(1)$ & $34(1)$ & $29(1)$ & $-4(1)$ & $-9(1)$ & $5(1)$ \\
\hline $\mathrm{O}(2)$ & $36(1)$ & $41(1)$ & $36(1)$ & $-11(1)$ & $-12(1)$ & $6(1)$ \\
\hline $\mathrm{O}(3)$ & $24(1)$ & $32(1)$ & $30(1)$ & $-4(1)$ & $-9(1)$ & $4(1)$ \\
\hline $\mathrm{N}(1)$ & $28(1)$ & $30(1)$ & $28(1)$ & $0(1)$ & $-8(1)$ & $-2(1)$ \\
\hline $\mathrm{C}(1)$ & $31(1)$ & $28(1)$ & $31(1)$ & $1(1)$ & $-2(1)$ & $1(1)$ \\
\hline $\mathrm{C}(2)$ & $26(1)$ & $30(1)$ & $27(1)$ & $4(1)$ & $-2(1)$ & $0(1)$ \\
\hline $\mathrm{C}(3)$ & $24(1)$ & $29(1)$ & $22(1)$ & $3(1)$ & $-4(1)$ & $-1(1)$ \\
\hline $\mathrm{C}(4)$ & $25(1)$ & $28(1)$ & $24(1)$ & $2(1)$ & $-4(1)$ & $-1(1)$ \\
\hline $\mathrm{C}(5)$ & $29(1)$ & $40(1)$ & $37(1)$ & $0(1)$ & $-5(1)$ & $8(1)$ \\
\hline $\mathrm{C}(6)$ & $24(1)$ & $29(1)$ & $24(1)$ & $2(1)$ & $-5(1)$ & $-2(1)$ \\
\hline $\mathrm{C}(7)$ & $23(1)$ & $32(1)$ & $31(1)$ & $0(1)$ & $-4(1)$ & $3(1)$ \\
\hline $\mathrm{C}(8)$ & $24(1)$ & $37(1)$ & $31(1)$ & $3(1)$ & $-6(1)$ & $-2(1)$
\end{tabular}


Table 5. Hydrogen coordinates ( $\left.\mathrm{x} 10^{\wedge} 4\right)$ and isotropic displacement parameters $\left(\mathrm{A}^{\wedge} 2 \mathrm{x}\right.$ $10^{\wedge} 3$ ) for compound 1.

\begin{tabular}{c|c|c|c|c} 
& $\mathrm{x}$ & $\mathrm{y}$ & $\mathrm{z}$ & $\mathrm{U}(\mathrm{eq})$ \\
\hline $\mathrm{H}(1)$ & 2454 & 830 & 6880 & 36 \\
\hline $\mathrm{H}(1 \mathrm{~N})$ & $-460(20)$ & $1876(13)$ & $6488(16)$ & 35 \\
\hline $\mathrm{H}(1 \mathrm{O})$ & $1820(20)$ & $4055(14)$ & $9995(15)$ & 37 \\
\hline $\mathrm{H}(5 \mathrm{~A})$ & $4980(20)$ & $1198(14)$ & $9919(17)$ & 43 \\
\hline $\mathrm{H}(5 \mathrm{~B})$ & $5950(20)$ & $2124(14)$ & $9209(16)$ & 43 \\
\hline $\mathrm{H}(5 \mathrm{C})$ & $5610(20)$ & $1046(14)$ & $8442(17)$ & 43 \\
\hline $\mathrm{H}(7 \mathrm{~A})$ & -3752 & 5027 & 6962 & 35 \\
\hline $\mathrm{H}(7 \mathrm{~B})$ & -4699 & 4225 & 7975 & 35 \\
\hline $\mathrm{H}(8 \mathrm{~A})$ & -5884 & 3114 & 6160 & 37 \\
\hline $\mathrm{H}(8 \mathrm{~B})$ & -4949 & 3917 & 5154 & 37 \\
\hline $\mathrm{H}(8 \mathrm{C})$ & -6632 & 4339 & 5966 & 37
\end{tabular}

Table 6. Torsion angles [deg] for compound $\mathbf{1}$.

\begin{tabular}{c|c}
$\begin{array}{c}\mathrm{C}(7)-\mathrm{O}(3)-\mathrm{C}(6)- \\
\mathrm{C}(4)\end{array}$ & $178.13(9)$ \\
\hline $\mathrm{C}(6)-\mathrm{O}(3)-\mathrm{C}(7)-\mathrm{C}(8)$ & $-178.20(9)$ \\
\hline $\mathrm{C}(7)-\mathrm{O}(3)-\mathrm{C}(6)-\mathrm{O}(2)$ & $-2.31(15)$ \\
\hline $\mathrm{C}(1)-\mathrm{N}(1)-\mathrm{C}(4)-\mathrm{C}(6)$ & $-179.46(10)$ \\
\hline $\mathrm{C}(1)-\mathrm{N}(1)-\mathrm{C}(4)-\mathrm{C}(3)$ & $-0.51(12)$ \\
\hline $\mathrm{C}(4)-\mathrm{N}(1)-\mathrm{C}(1)-\mathrm{C}(2)$ & $0.69(13)$ \\
\hline $\mathrm{N}(1)-\mathrm{C}(1)-\mathrm{C}(2)-\mathrm{C}(5)$ & $180.00(11)$ \\
\hline $\mathrm{N}(1)-\mathrm{C}(1)-\mathrm{C}(2)-\mathrm{C}(3)$ & $-0.58(13)$ \\
\hline $\mathrm{C}(5)-\mathrm{C}(2)-\mathrm{C}(3)-\mathrm{O}(1)$ & $0.45(19)$ \\
\hline $\mathrm{C}(5)-\mathrm{C}(2)-\mathrm{C}(3)-\mathrm{C}(4)$ & $179.67(11)$ \\
\hline $\mathrm{C}(1)-\mathrm{C}(2)-\mathrm{C}(3)-\mathrm{C}(4)$ & $0.25(13)$ \\
\hline $\mathrm{C}(1)-\mathrm{C}(2)-\mathrm{C}(3)-\mathrm{O}(1)$ & $-178.97(10)$ \\
\hline $\mathrm{O}(1)-\mathrm{C}(3)-\mathrm{C}(4)-\mathrm{C}(6)$ & $-1.74(19)$ \\
\hline $\mathrm{C}(2)-\mathrm{C}(3)-\mathrm{C}(4)-\mathrm{N}(1)$ & $0.15(12)$ \\
\hline $\mathrm{C}(2)-\mathrm{C}(3)-\mathrm{C}(4)-\mathrm{C}(6)$ & $179.07(11)$ \\
\hline $\mathrm{O}(1)-\mathrm{C}(3)-\mathrm{C}(4)-\mathrm{N}(1)$ & $179.34(10)$ \\
\hline $\mathrm{C}(3)-\mathrm{C}(4)-\mathrm{C}(6)-\mathrm{O}(3)$ & $179.83(10)$ \\
\hline $\mathrm{C}(3)-\mathrm{C}(4)-\mathrm{C}(6)-\mathrm{O}(2)$ & $0.27(18)$ \\
\hline $\mathrm{N}(1)-\mathrm{C}(4)-\mathrm{C}(6)-\mathrm{O}(2)$ & $179.00(11)$ \\
&
\end{tabular}


$\mathrm{N}(1)-\mathrm{C}(4)-\mathrm{C}(6)-\mathrm{O}(3) \mid-1.44(15)$

Table 7. Hydrogen-bonds for compound 1 [A and deg.].

\begin{tabular}{c|c|c|c|c}
$\mathrm{D}-\mathrm{H} \ldots \mathrm{A}$ & $\mathrm{d}(\mathrm{D}-\mathrm{H})$ & $\mathrm{d}(\mathrm{H} \ldots \mathrm{A})$ & $\mathrm{d}(\mathrm{D} \ldots \mathrm{A})$ & $<(\mathrm{DHA})$ \\
\hline & & & & \\
\hline $\mathrm{N}(1)-\mathrm{H}(1 \mathrm{~N}) \ldots \mathrm{O}(1) \# 1$ & $0.891(15)$ & $2.094(15)$ & $2.9796(13)$ & $172.4(14)$ \\
\hline $\mathrm{O}(1)-\mathrm{H}(1 \mathrm{O}) \ldots \mathrm{O}(2)$ & $0.855(16)$ & $2.330(15)$ & $2.9747(12)$ & $132.4(13)$ \\
\hline $\mathrm{O}(1)-\mathrm{H}(1 \mathrm{O}) \ldots \mathrm{O}(2) \# 2$ & $0.855(16)$ & $2.174(16)$ & $2.9258(13)$ & $146.5(13)$
\end{tabular}

Symmetry transformations used to generate equivalent atoms:

\#1 1/2+x-1,1/2-y,1/2+z-1 \#2 -x,-y+1,-z+2 


\section{3-Methoxy-4-methyl-1H-pyrrole-2-carboxylic acid ethyl ester, 2.}

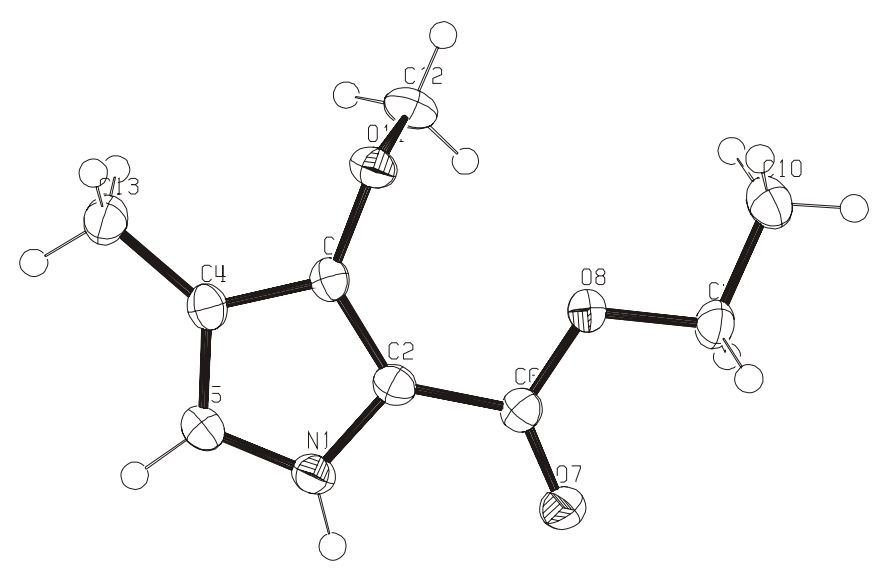

Table 1. Crystal data and structure refinement for compound 2.

\begin{tabular}{|c|c|}
\hline Crystal Data & \\
\hline Empirical formula & C9 H13 N O3 \\
\hline Formula weight & 183.20 \\
\hline Crystal size & $0.44 \times 0.28 \times 0.10 \mathrm{~mm}$ \\
\hline Crystal description & platelike rod \\
\hline Crystal colour & translucent colourless \\
\hline Crystal system & Monoclinic \\
\hline Space group & P 21 \\
\hline \multirow[t]{3}{*}{ Unit cell dimensions } & $\mathrm{a}=7.8615(9)$ A alpha $=90 \mathrm{deg}$. \\
\hline & $\mathrm{b}=6.9727(5) \mathrm{A} \quad$ beta $=91.877(13) \mathrm{deg}$. \\
\hline & $\mathrm{c}=8.5838(9) \mathrm{A}$ gamma $=90 \mathrm{deg}$ \\
\hline Volume & $470.28(8) \mathrm{A}^{\wedge} 3$ \\
\hline Z, Calculated density & $2,1.294 \mathrm{Mg} / \mathrm{m}^{\wedge} 3$ \\
\hline Absorption coefficient & $0.097 \mathrm{~mm}^{\wedge}-1$ \\
\hline $\mathrm{F}(000)$ & 196 \\
\hline \multicolumn{2}{|l|}{ Data Collection } \\
\hline Measurement device type & STOE-IPDS diffractometer \\
\hline Measuremnet method & rotation \\
\hline Temperature & $173(1) \mathrm{K}$ \\
\hline Wavelength & $0.71073 \mathrm{~A}$ \\
\hline Monochromator & graphite \\
\hline Theta range for data collection & 2.37 to $27.89 \mathrm{deg}$. \\
\hline Index ranges & $-10<=\mathrm{h}<=10,-9<=\mathrm{k}<=9,-11<=\mathrm{l}<=11$ \\
\hline
\end{tabular}




\begin{tabular}{c|c} 
Reflections collected / unique & $7765 / 2222[\mathrm{R}(\mathrm{int})=0.0900]$ \\
\hline Reflections greater I $>4 \backslash \mathrm{l}(\mathrm{I})$ & 2021 \\
\hline Absorption correction & None \\
\hline Refinement & Full-matrix least-squares on $\mathrm{F}^{\wedge} 2$ \\
\hline Refinement method & refall \\
\hline Hydrogen treatment & $2222 / 1 / 170$ \\
\hline Data / restraints / parameters & $\mathrm{R} 1=0.0347, \mathrm{wR} 2=0.0825$ \\
\hline Goodness-of-fit on F^2 & $\mathrm{R} 1=0.0393, \mathrm{wR} 2=0.0846$ \\
\hline Final R indices [I $>2$ sigma(I)] & $0.0(8)$ \\
\hline R indices (all data) & 0.292 and $-0.134 \mathrm{e} . \mathrm{A}^{\wedge}-3$
\end{tabular}

Table 2. Atomic coordinates ( $\times 10^{\wedge} 4$ ) and equivalent isotropic displacement parameters $\left(\mathrm{A}^{\wedge} 2 \times 10^{\wedge} 3\right)$ for compound 2 . $\mathrm{U}(\mathrm{eq})$ is defined as one third of the trace of the orthogonalized Uij tensor.

\begin{tabular}{c|c|c|c|c} 
& $\mathrm{x}$ & $\mathrm{y}$ & $\mathrm{z}$ & $\mathrm{U}(\mathrm{eq})$ \\
\hline $\mathrm{O}(7)$ & $10056(1)$ & $3154(2)$ & $6060(1)$ & $32(1)$ \\
\hline $\mathrm{O}(8)$ & $8450(1)$ & $4666(1)$ & $7821(1)$ & $27(1)$ \\
\hline $\mathrm{O}(11)$ & $5370(1)$ & $2761(1)$ & $8750(1)$ & $26(1)$ \\
\hline $\mathrm{N}(1)$ & $7514(2)$ & $262(2)$ & $5781(1)$ & $26(1)$ \\
\hline $\mathrm{C}(2)$ & $7445(2)$ & $1792(2)$ & $6795(2)$ & $23(1)$ \\
\hline $\mathrm{C}(3)$ & $5914(2)$ & $1634(2)$ & $7563(2)$ & $21(1)$ \\
\hline $\mathrm{C}(4)$ & $5036(2)$ & $-2(2)$ & $6978(2)$ & $24(1)$ \\
\hline $\mathrm{C}(5)$ & $6085(2)$ & $-807(2)$ & $5893(2)$ & $26(1)$ \\
\hline $\mathrm{C}(6)$ & $8787(2)$ & $3228(2)$ & $6835(2)$ & $23(1)$ \\
\hline $\mathrm{C}(9)$ & $9670(2)$ & $6232(2)$ & $7909(2)$ & $29(1)$ \\
\hline $\mathrm{C}(10)$ & $9034(2)$ & $7633(2)$ & $9087(2)$ & $34(1)$ \\
\hline $\mathrm{C}(12)$ & $4467(2)$ & $4452(2)$ & $8260(2)$ & $32(1)$ \\
\hline $\mathrm{C}(13)$ & $3313(2)$ & $-681(2)$ & $7443(2)$ & $31(1)$ \\
& & & &
\end{tabular}

Table 3. Bond lengths [A] and angles [deg] for compound 2.

\begin{tabular}{c|c}
$\mathrm{O}(7)-\mathrm{C}(6)$ & $1.2188(17)$ \\
\hline $\mathrm{O}(8)-\mathrm{C}(6)$ & $1.3438(16)$ \\
\hline $\mathrm{O}(8)-\mathrm{C}(9)$ & $1.4534(17)$ \\
\hline $\mathrm{O}(11)-\mathrm{C}(3)$ & $1.3663(16)$
\end{tabular}




\begin{tabular}{|c|c|}
\hline $\mathrm{O}(11)-\mathrm{C}(12)$ & $1.4324(18)$ \\
\hline $\mathrm{N}(1)-\mathrm{C}(2)$ & $1.3787(18)$ \\
\hline $\mathrm{N}(1)-\mathrm{C}(5)$ & $1.3546(18)$ \\
\hline $\mathrm{N}(1)-\mathrm{H}(1)$ & $0.866(16)$ \\
\hline$C(2)-C(6)$ & $1.4540(19)$ \\
\hline $\mathrm{C}(2)-\mathrm{C}(3)$ & $1.3955(18)$ \\
\hline$C(3)-C(4)$ & $1.4166(19)$ \\
\hline$C(4)-C(13)$ & $1.501(2)$ \\
\hline $\mathrm{C}(4)-\mathrm{C}(5)$ & $1.3832(19)$ \\
\hline$C(9)-C(10)$ & $1.504(2)$ \\
\hline $\mathrm{C}(5)-\mathrm{H}(5)$ & $0.95(2)$ \\
\hline $\mathrm{C}(9)-\mathrm{H}(9 \mathrm{~A})$ & $0.98(2)$ \\
\hline $\mathrm{C}(9)-\mathrm{H}(9 \mathrm{~B})$ & $0.973(18)$ \\
\hline $\mathrm{C}(10)-\mathrm{H}(10 \mathrm{~A})$ & $0.95(2)$ \\
\hline $\mathrm{C}(10)-\mathrm{H}(10 \mathrm{~B})$ & $0.997(18)$ \\
\hline $\mathrm{C}(10)-\mathrm{H}(10 \mathrm{C})$ & $0.95(3)$ \\
\hline $\mathrm{C}(12)-\mathrm{H}(12 \mathrm{~A})$ & $1.01(2)$ \\
\hline $\mathrm{C}(12)-\mathrm{H}(12 \mathrm{~B})$ & $0.934(18)$ \\
\hline $\mathrm{C}(12)-\mathrm{H}(12 \mathrm{C})$ & $0.98(2)$ \\
\hline $\mathrm{C}(13)-\mathrm{H}(13 \mathrm{~A})$ & $0.941(17)$ \\
\hline $\mathrm{C}(13)-\mathrm{H}(13 \mathrm{~B})$ & $0.93(3)$ \\
\hline $\mathrm{C}(13)-\mathrm{H}(13 \mathrm{C})$ & $0.96(2)$ \\
\hline $\mathrm{C}(6)-\mathrm{O}(8)-\mathrm{C}(9)$ & $116.74(11)$ \\
\hline $\mathrm{C}(3)-\mathrm{O}(11)-\mathrm{C}(12)$ & $114.76(11)$ \\
\hline $\mathrm{C}(2)-\mathrm{N}(1)-\mathrm{C}(5)$ & $109.34(11)$ \\
\hline $\mathrm{C}(5)-\mathrm{N}(1)-\mathrm{H}(1)$ & $127.0(13)$ \\
\hline $\mathrm{C}(2)-\mathrm{N}(1)-\mathrm{H}(1)$ & $123.7(13)$ \\
\hline$C(3)-C(2)-C(6)$ & $132.79(12)$ \\
\hline $\mathrm{N}(1)-\mathrm{C}(2)-\mathrm{C}(6)$ & $120.28(12)$ \\
\hline $\mathrm{N}(1)-\mathrm{C}(2)-\mathrm{C}(3)$ & $106.83(11)$ \\
\hline $\mathrm{O}(11)-\mathrm{C}(3)-\mathrm{C}(2)$ & $127.06(12)$ \\
\hline $\mathrm{O}(11)-\mathrm{C}(3)-\mathrm{C}(4)$ & $124.52(11)$ \\
\hline $\mathrm{C}(2)-\mathrm{C}(3)-\mathrm{C}(4)$ & $108.30(11)$ \\
\hline $\mathrm{C}(3)-\mathrm{C}(4)-\mathrm{C}(5)$ & $105.73(11)$ \\
\hline$C(5)-C(4)-C(13)$ & $127.88(13)$ \\
\hline $\mathrm{C}(3)-\mathrm{C}(4)-\mathrm{C}(13)$ & $126.39(12)$ \\
\hline $\mathrm{N}(1)-\mathrm{C}(5)-\mathrm{C}(4)$ & $109.79(12)$ \\
\hline $\mathrm{O}(7)-\mathrm{C}(6)-\mathrm{O}(8)$ & $123.85(12)$ \\
\hline $\mathrm{O}(7)-\mathrm{C}(6)-\mathrm{C}(2)$ & $124.35(13)$ \\
\hline
\end{tabular}




\begin{tabular}{|c|c|}
\hline $\mathrm{O}(8)-\mathrm{C}(6)-\mathrm{C}(2)$ & $111.80(11)$ \\
\hline $\mathrm{O}(8)-\mathrm{C}(9)-\mathrm{C}(10)$ & $106.83(12)$ \\
\hline $\mathrm{N}(1)-\mathrm{C}(5)-\mathrm{H}(5)$ & $122.0(10)$ \\
\hline $\mathrm{C}(4)-\mathrm{C}(5)-\mathrm{H}(5)$ & $128.2(10)$ \\
\hline $\mathrm{O}(8)-\mathrm{C}(9)-\mathrm{H}(9 \mathrm{~A})$ & $108.5(13)$ \\
\hline $\mathrm{O}(8)-\mathrm{C}(9)-\mathrm{H}(9 \mathrm{~B})$ & $110.4(11)$ \\
\hline $\mathrm{C}(10)-\mathrm{C}(9)-\mathrm{H}(9 \mathrm{~A})$ & 111.4(11) \\
\hline $\mathrm{C}(10)-\mathrm{C}(9)-\mathrm{H}(9 \mathrm{~B})$ & $109.6(12)$ \\
\hline $\mathrm{H}(9 \mathrm{~A})-\mathrm{C}(9)-\mathrm{H}(9 \mathrm{~B})$ & $110.0(15)$ \\
\hline $\mathrm{C}(9)-\mathrm{C}(10)-\mathrm{H}(10 \mathrm{~A})$ & $112.6(11)$ \\
\hline $\mathrm{C}(9)-\mathrm{C}(10)-\mathrm{H}(10 \mathrm{~B})$ & $114.0(12)$ \\
\hline $\mathrm{C}(9)-\mathrm{C}(10)-\mathrm{H}(10 \mathrm{C})$ & $109.4(15)$ \\
\hline $\mathrm{H}(10 \mathrm{~A})-\mathrm{C}(10)-\mathrm{H}(10 \mathrm{~B})$ & $101.8(15)$ \\
\hline $\mathrm{H}(10 \mathrm{~A})-\mathrm{C}(10)-\mathrm{H}(10 \mathrm{C})$ & $116(2)$ \\
\hline $\mathrm{H}(10 \mathrm{~B})-\mathrm{C}(10)-\mathrm{H}(10 \mathrm{C})$ & 103.1(19) \\
\hline $\mathrm{O}(11)-\mathrm{C}(12)-\mathrm{H}(12 \mathrm{~A})$ & $107.0(15)$ \\
\hline $\mathrm{O}(11)-\mathrm{C}(12)-\mathrm{H}(12 \mathrm{~B})$ & $109.8(11)$ \\
\hline $\mathrm{O}(11)-\mathrm{C}(12)-\mathrm{H}(12 \mathrm{C})$ & $111.8(16)$ \\
\hline $\mathrm{H}(12 \mathrm{~A})-\mathrm{C}(12)-\mathrm{H}(12 \mathrm{~B})$ & $113.8(18)$ \\
\hline $\mathrm{H}(12 \mathrm{~A})-\mathrm{C}(12)-\mathrm{H}(12 \mathrm{C})$ & $107.5(17)$ \\
\hline $\mathrm{H}(12 \mathrm{~B})-\mathrm{C}(12)-\mathrm{H}(12 \mathrm{C})$ & $106.9(16)$ \\
\hline $\mathrm{C}(4)-\mathrm{C}(13)-\mathrm{H}(13 \mathrm{~A})$ & $113.0(10)$ \\
\hline $\mathrm{C}(4)-\mathrm{C}(13)-\mathrm{H}(13 \mathrm{~B})$ & $113.0(16)$ \\
\hline $\mathrm{C}(4)-\mathrm{C}(13)-\mathrm{H}(13 \mathrm{C})$ & $108.8(13)$ \\
\hline $\mathrm{H}(13 \mathrm{~A})-\mathrm{C}(13)-\mathrm{H}(13 \mathrm{~B})$ & 107.4(19) \\
\hline $\mathrm{H}(13 \mathrm{~A})-\mathrm{C}(13)-\mathrm{H}(13 \mathrm{C})$ & 109.1(17) \\
\hline $\mathrm{H}(13 \mathrm{~B})-\mathrm{C}(13)-\mathrm{H}(13 \mathrm{C})$ & $105(2)$ \\
\hline
\end{tabular}

Table 4. Anisotropic displacement parameters $\left(\mathrm{A}^{\wedge} 2 \times 10^{\wedge} 3\right)$ for compound 2 . The anisotropic displacement factor exponent takes the form: $-2 \mathrm{pi}^{\wedge} 2\left[\mathrm{~h}^{\wedge} 2 \mathrm{a}^{* \wedge} 2 \mathrm{U} 11+\ldots+2 \mathrm{~h} \mathrm{k} \mathrm{a*} \mathrm{b}^{*} \mathrm{U} 12\right.$ ]

\begin{tabular}{c|c|c|c|c|c|c} 
& $\mathrm{U} 11$ & $\mathrm{U} 22$ & $\mathrm{U} 33$ & $\mathrm{U} 23$ & $\mathrm{U} 13$ & $\mathrm{U} 12$ \\
\hline & & & & & & \\
\hline $\mathrm{O}(7)$ & $31(1)$ & $27(1)$ & $37(1)$ & $-2(1)$ & $11(1)$ & $-3(1)$ \\
\hline $\mathrm{O}(8)$ & $25(1)$ & $23(1)$ & $34(1)$ & $-5(1)$ & $4(1)$ & $-4(1)$ \\
\hline $\mathrm{O}(11)$ & $32(1)$ & $23(1)$ & $23(1)$ & $-1(1)$ & $4(1)$ & $3(1)$ \\
\hline $\mathrm{N}(1)$ & $30(1)$ & $22(1)$ & $25(1)$ & $-2(1)$ & $6(1)$ & $1(1)$ \\
\hline $\mathrm{C}(2)$ & $26(1)$ & $21(1)$ & $22(1)$ & $1(1)$ & $0(1)$ & $3(1)$ \\
\hline $\mathrm{C}(3)$ & $25(1)$ & $19(1)$ & $21(1)$ & $3(1)$ & $-1(1)$ & $-1(1)$ \\
\hline $\mathrm{C}(4)$ & $27(1)$ & $20(1)$ & $25(1)$ & $2(1)$ & $-2(1)$ & $-1(1)$
\end{tabular}




\begin{tabular}{c|c|c|c|c|c|c}
$\mathrm{C}(5)$ & $31(1)$ & $21(1)$ & $24(1)$ & $-1(1)$ & $0(1)$ & $1(1)$ \\
\hline $\mathrm{C}(6)$ & $25(1)$ & $21(1)$ & $24(1)$ & $4(1)$ & $1(1)$ & $0(1)$ \\
\hline $\mathrm{C}(9)$ & $26(1)$ & $23(1)$ & $37(1)$ & $1(1)$ & $1(1)$ & $-5(1)$ \\
\hline $\mathrm{C}(10)$ & $40(1)$ & $27(1)$ & $35(1)$ & $-4(1)$ & $0(1)$ & $-4(1)$ \\
\hline $\mathrm{C}(12)$ & $35(1)$ & $30(1)$ & $33(1)$ & $-5(1)$ & $2(1)$ & $11(1)$ \\
\hline $\mathrm{C}(13)$ & $27(1)$ & $30(1)$ & $36(1)$ & $0(1)$ & $1(1)$ & $-4(1)$
\end{tabular}

Table 5. Hydrogen coordinates ( $\left.x 10^{\wedge} 4\right)$ and isotropic displacement parameters $\left(\mathrm{A}^{\wedge} 2 \mathrm{x}\right.$ $10^{\wedge} 3$ ) for compound 2.

\begin{tabular}{c|c|c|c|c} 
& $\mathrm{x}$ & $\mathrm{y}$ & $\mathrm{z}$ & $\mathrm{U}(\mathrm{eq})$ \\
\hline & & & & \\
\hline $\mathrm{H}(1)$ & $8350(20)$ & $60(30)$ & $5170(20)$ & $24(4)$ \\
\hline $\mathrm{H}(5)$ & $5900(20)$ & $-1940(30)$ & $5290(20)$ & $34(5)$ \\
\hline $\mathrm{H}(9 \mathrm{~A})$ & $10780(30)$ & $5710(30)$ & $8230(20)$ & $39(5)$ \\
\hline $\mathrm{H}(9 \mathrm{~B})$ & $9740(20)$ & $6860(30)$ & $6900(20)$ & $39(5)$ \\
\hline $\mathrm{H}(10 \mathrm{~A})$ & $7910(30)$ & $8050(30)$ & $8850(20)$ & $40(5)$ \\
\hline $\mathrm{H}(10 \mathrm{~B})$ & $8920(20)$ & $7080(30)$ & $10150(20)$ & $35(5)$ \\
\hline $\mathrm{H}(10 \mathrm{C})$ & $9850(30)$ & $8620(40)$ & $9250(30)$ & $62(7)$ \\
\hline $\mathrm{H}(12 \mathrm{~A})$ & $5210(30)$ & $5150(40)$ & $7510(20)$ & $45(5)$ \\
\hline $\mathrm{H}(12 \mathrm{~B})$ & $4190(20)$ & $5180(30)$ & $9130(20)$ & $35(5)$ \\
\hline $\mathrm{H}(12 \mathrm{C})$ & $3390(30)$ & $4140(40)$ & $7700(20)$ & $48(6)$ \\
\hline $\mathrm{H}(13 \mathrm{~A})$ & $3270(20)$ & $-980(30)$ & $8510(20)$ & $38(5)$ \\
\hline $\mathrm{H}(13 \mathrm{~B})$ & $2460(30)$ & $200(40)$ & $7220(30)$ & $53(6)$ \\
\hline $\mathrm{H}(13 \mathrm{C})$ & $3010(30)$ & $-1800(30)$ & $6840(20)$ & $39(5)$
\end{tabular}

Table 6. Torsion angles [deg] for compound 2.

\begin{tabular}{c|c}
$\mathrm{C}(6)-\mathrm{O}(8)-\mathrm{C}(9)-\mathrm{C}(10)$ & $-179.34(11)$ \\
\hline $\mathrm{C}(9)-\mathrm{O}(8)-\mathrm{C}(6)-\mathrm{O}(7)$ & $1.89(19)$ \\
\hline $\mathrm{C}(9)-\mathrm{O}(8)-\mathrm{C}(6)-\mathrm{C}(2)$ & $-177.44(11)$ \\
\hline $\mathrm{C}(12)-\mathrm{O}(11)-\mathrm{C}(3)-\mathrm{C}(2)$ & $87.71(16)$ \\
\hline $\mathrm{C}(12)-\mathrm{O}(11)-\mathrm{C}(3)-\mathrm{C}(4)$ & $-96.95(15)$ \\
\hline $\mathrm{C}(2)-\mathrm{N}(1)-\mathrm{C}(5)-\mathrm{C}(4)$ & $0.25(16)$ \\
\hline $\mathrm{C}(5)-\mathrm{N}(1)-\mathrm{C}(2)-\mathrm{C}(3)$ & $0.50(15)$ \\
\hline $\mathrm{C}(5)-\mathrm{N}(1)-\mathrm{C}(2)-\mathrm{C}(6)$ & $-176.40(12)$ \\
\hline $\mathrm{N}(1)-\mathrm{C}(2)-\mathrm{C}(6)-\mathrm{O}(7)$ & $-3.2(2)$ \\
\hline $\mathrm{C}(3)-\mathrm{C}(2)-\mathrm{C}(6)-\mathrm{O}(8)$ & $0.2(2)$ \\
\hline $\mathrm{N}(1)-\mathrm{C}(2)-\mathrm{C}(3)-\mathrm{C}(4)$ & $-1.04(15)$
\end{tabular}




\begin{tabular}{c|c}
$\mathrm{C}(6)-\mathrm{C}(2)-\mathrm{C}(3)-\mathrm{O}(11)$ & $-8.7(2)$ \\
\hline $\mathrm{N}(1)-\mathrm{C}(2)-\mathrm{C}(3)-\mathrm{O}(11)$ & $174.91(12)$ \\
\hline $\mathrm{C}(3)-\mathrm{C}(2)-\mathrm{C}(6)-\mathrm{O}(7)$ & $-179.11(14)$ \\
\hline $\mathrm{N}(1)-\mathrm{C}(2)-\mathrm{C}(6)-\mathrm{O}(8)$ & $176.17(11)$ \\
\hline $\mathrm{C}(6)-\mathrm{C}(2)-\mathrm{C}(3)-\mathrm{C}(4)$ & $175.30(14)$ \\
\hline $\mathrm{C}(2)-\mathrm{C}(3)-\mathrm{C}(4)-\mathrm{C}(13)$ & $-178.12(13)$ \\
\hline $\mathrm{O}(11)-\mathrm{C}(3)-\mathrm{C}(4)-\mathrm{C}(13)$ & $5.8(2)$ \\
\hline $\mathrm{C}(2)-\mathrm{C}(3)-\mathrm{C}(4)-\mathrm{C}(5)$ & $1.18(15)$ \\
\hline $\mathrm{O}(11)-\mathrm{C}(3)-\mathrm{C}(4)-\mathrm{C}(5)$ & $-174.90(12)$ \\
\hline $\mathrm{C}(3)-\mathrm{C}(4)-\mathrm{C}(5)-\mathrm{N}(1)$ & $-0.88(15)$ \\
\hline $\mathrm{C}(13)-\mathrm{C}(4)-\mathrm{C}(5)-\mathrm{N}(1)$ & $178.40(13)$
\end{tabular}

Table 7. Hydrogen-bonds for compound 2 [A and deg.].

\begin{tabular}{c|c|c|c|c}
$\mathrm{D}-\mathrm{H} \ldots \mathrm{A}$ & $\mathrm{d}(\mathrm{D}-\mathrm{H})$ & $\mathrm{d}(\mathrm{H} \ldots \mathrm{A})$ & $\mathrm{d}(\mathrm{D} \ldots \mathrm{A})$ & $<(\mathrm{DHA})$ \\
\hline & & & & \\
\hline $\mathrm{N}(1)-\mathrm{H}(1) \ldots \mathrm{O}(7) \# 1$ & $0.866(16)$ & $2.130(18)$ & $2.9163(16)$ & $150.7(19)$ \\
\hline $\mathrm{C}(12)-\mathrm{H}(12 \mathrm{~A}) \ldots \mathrm{O}(8)$ & $1.01(2)$ & $2.57(2)$ & $3.1697(19)$ & $117.5(16)$ \\
\hline $\mathrm{C}(12)-\mathrm{H}(12 \mathrm{~B}) \ldots \mathrm{O}(11) \# 2$ & $0.934(18)$ & $2.575(19)$ & $3.4503(18)$ & $156.2(14)$
\end{tabular}

Symmetry transformations used to generate equivalent atoms:

$\# 1-\mathrm{x}+2,1 / 2+\mathrm{y}-1,-\mathrm{z}+1 \quad \# 2-\mathrm{x}+1,1 / 2+\mathrm{y},-\mathrm{z}+2$ 


\section{Ethyl 5-formyl-3-methoxy-4-methyl-1H-pyrrole-2-carboxylate, 3.}

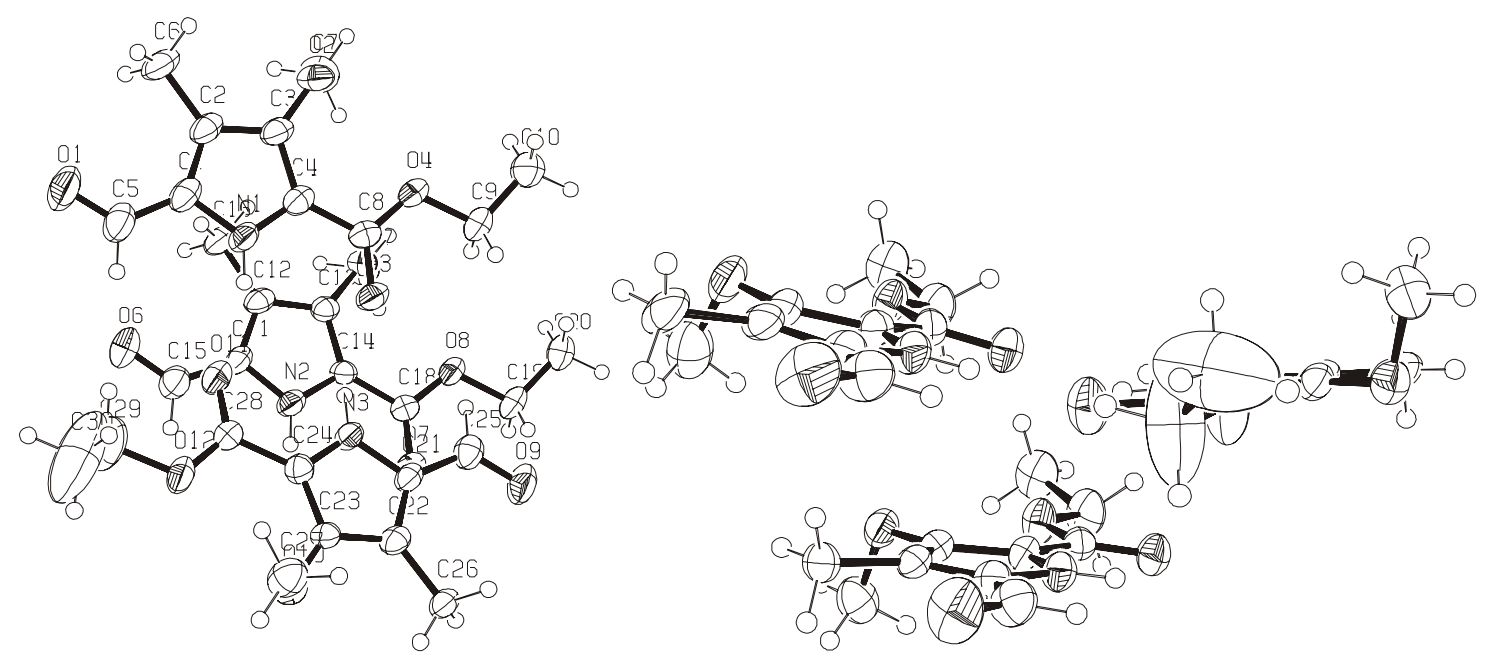

Table 1. Crystal data and structure refinement for compound $\mathbf{3}$.

\begin{tabular}{|c|c|}
\hline Crystal Data & \\
\hline Empirical formula & C10 H13 N O4 \\
\hline Formula weight & 211.21 \\
\hline Crystal size & $0.32 \times 0.28 \times 0.20 \mathrm{~mm}$ \\
\hline Crystal description & prism \\
\hline Crystal colour & colourless \\
\hline Crystal system & Triclinic \\
\hline Space group & $\mathrm{P}-1$ \\
\hline \multirow[t]{3}{*}{ Unit cell dimensions } & $\mathrm{a}=11.3701(12)$ A alpha $=69.719(12) \mathrm{deg}$. \\
\hline & $\mathrm{b}=12.0463(13) \mathrm{A} \quad$ beta $=70.868(13) \mathrm{deg}$. \\
\hline & $\mathrm{c}=14.0990(16) \mathrm{A} \quad$ gamma $=63.879(12) \mathrm{deg}$. \\
\hline Volume & $1590.8(4) \mathrm{A}^{\wedge} 3$ \\
\hline Z, Calculated density & $6,1.323 \mathrm{Mg} / \mathrm{m}^{\wedge} 3$ \\
\hline Absorption coefficient & $0.103 \mathrm{~mm}^{\wedge}-1$ \\
\hline $\mathrm{F}(000)$ & 672 \\
\hline \multicolumn{2}{|l|}{ Data Collection } \\
\hline Measurement device type & STOE-IPDS diffractometer \\
\hline Measuremnet method & rotation \\
\hline Temperature & $173(1) \mathrm{K}$ \\
\hline Wavelength & $0.71073 \mathrm{~A}$ \\
\hline Monochromator & graphite \\
\hline Theta range for data collection & 1.94 to $25.79 \mathrm{deg}$. \\
\hline
\end{tabular}




\begin{tabular}{c|c} 
Index ranges & $-13<=\mathrm{h}<=13,-14<=\mathrm{k}<=14,-17<=\mathrm{l}<=17$ \\
\hline Reflections collected / unique & $11929 / 5712[\mathrm{R}(\mathrm{int})=0.1081]$ \\
\hline Reflections greater I $>4 \backslash \mathrm{ls}(\mathrm{I})$ & 2501 \\
\hline Absorption correction & None \\
\hline Refinement & Full-matrix least-squares on $\mathrm{F}^{\wedge} 2$ \\
\hline Refinement method & mixed \\
\hline Hydrogen treatment & 5712 / 0 / 406 \\
\hline Data / restraints / parameters & 0.818 \\
\hline Goodness-of-fit on F^2 & $\mathrm{R} 1=0.0663, \mathrm{wR} 2=0.1454$ \\
\hline Final R indices [I $>2$ sigma(I)] & $\mathrm{R} 1=0.1439, \mathrm{wR} 2=0.1734$ \\
\hline R indices (all data) & $?$ \\
\hline Absolute structure parameter & 0.655 and $-0.292 \mathrm{e} . \mathrm{A}^{\wedge}-3$
\end{tabular}

Table 2. Atomic coordinates ( $\left.\mathrm{x} 10^{\wedge} 4\right)$ and equivalent isotropic displacement parameters $\left(\mathrm{A}^{\wedge} 2 \times 10^{\wedge} 3\right)$ for compound 3 . $\mathrm{U}(\mathrm{eq})$ is defined as one third of the trace of the orthogonalized Uij tensor.

\begin{tabular}{|c|c|c|c|c|}
\hline & $\mathrm{x}$ & $\mathrm{y}$ & Z & $\mathrm{U}(\mathrm{eq})$ \\
\hline $\mathrm{O}(1)$ & $8189(4)$ & $2237(3)$ & $6710(2)$ & $73(1)$ \\
\hline $\mathrm{O}(2)$ & $6849(3)$ & $7428(3)$ & $5681(2)$ & $53(1)$ \\
\hline $\mathrm{O}(3)$ & 9591(3) & $6397(3)$ & $2785(2)$ & $42(1)$ \\
\hline $\mathrm{O}(4)$ & $8354(3)$ & $8032(3)$ & $3542(2)$ & $42(1)$ \\
\hline $\mathrm{N}(1)$ & $8843(3)$ & $4753(3)$ & $4606(2)$ & $38(1)$ \\
\hline $\mathrm{C}(1)$ & $8275(4)$ & $4170(4)$ & $5576(3)$ & $43(2)$ \\
\hline $\mathrm{C}(2)$ & $7435(4)$ & $5128(4)$ & $6098(3)$ & $44(2)$ \\
\hline $\mathrm{C}(3)$ & $7503(4)$ & $6279(4)$ & $5441(3)$ & $40(1)$ \\
\hline $\mathrm{C}(4)$ & $8397(4)$ & $6009(4)$ & $4506(3)$ & $36(1)$ \\
\hline$C(5)$ & $8610(5)$ & $2837(5)$ & $5864(3)$ & $54(2)$ \\
\hline$C(6)$ & $6561(5)$ & $4965(6)$ & $7176(3)$ & $64(2)$ \\
\hline $\mathrm{C}(7)$ & $5602(5)$ & $8116(5)$ & $5351(4)$ & $71(2)$ \\
\hline $\mathrm{C}(8)$ & $8841(4)$ & $6828(4)$ & $3528(3)$ & $35(1)$ \\
\hline $\mathrm{C}(9)$ & $8680(5)$ & $8912(4)$ & $2566(3)$ & $47(2)$ \\
\hline $\mathrm{C}(10)$ & $8216(5)$ & $10179(4)$ & 2771(3) & $57(2)$ \\
\hline $\mathrm{O}(5)$ & 6101(3) & $8067(3)$ & $2158(2)$ & $40(1)$ \\
\hline $\mathrm{O}(6)$ & $7559(4)$ & 2861(3) & $3638(2)$ & 69(1) \\
\hline $\mathrm{O}(7)$ & 9399(3) & $6675(3)$ & $-396(2)$ & $40(1)$ \\
\hline $\mathrm{O}(8)$ & $7963(3)$ & $8435(3)$ & $169(2)$ & $43(1)$ \\
\hline
\end{tabular}




\begin{tabular}{c|c|c|c|c}
$\mathrm{N}(2)$ & $8440(3)$ & $5192(3)$ & $1455(2)$ & $36(1)$ \\
\hline $\mathrm{C}(11)$ & $7739(4)$ & $4728(4)$ & $2403(2)$ & $36(1)$ \\
\hline $\mathrm{C}(12)$ & $6780(4)$ & $5744(4)$ & $2803(3)$ & $36(1)$ \\
\hline $\mathrm{C}(13)$ & $6885(4)$ & $6848(4)$ & $2063(3)$ & $33(1)$ \\
\hline $\mathrm{C}(14)$ & $7916(4)$ & $6482(3)$ & $1227(2)$ & $30(1)$ \\
\hline $\mathrm{C}(15)$ & $8101(5)$ & $3364(4)$ & $2802(3)$ & $48(2)$ \\
\hline $\mathrm{C}(16)$ & $5776(4)$ & $5704(5)$ & $3811(3)$ & $45(1)$ \\
\hline $\mathrm{C}(17)$ & $4973(4)$ & $8616(4)$ & $1672(3)$ & $54(2)$ \\
\hline $\mathrm{C}(18)$ & $8501(4)$ & $7191(4)$ & $253(3)$ & $32(1)$ \\
\hline $\mathrm{C}(19)$ & $8455(5)$ & $9231(4)$ & $-794(3)$ & $53(2)$ \\
\hline $\mathrm{C}(20)$ & $7954(5)$ & $10526(4)$ & $-641(3)$ & $57(2)$ \\
\hline $\mathrm{O}(9)$ & $12249(3)$ & $7092(3)$ & $-512(2)$ & $55(1)$ \\
\hline $\mathrm{O}(10)$ & $14357(3)$ & $1868(3)$ & $1035(2)$ & $42(1)$ \\
\hline $\mathrm{O}(11)$ & $10645(3)$ & $3026(3)$ & $3390(2)$ & $50(1)$ \\
\hline $\mathrm{O}(12)$ & $12397(3)$ & $1347(3)$ & $2931(2)$ & $64(1)$ \\
\hline $\mathrm{N}(3)$ & $11578(3)$ & $4612(3)$ & $1627(2)$ & $33(1)$ \\
\hline $\mathrm{C}(21)$ & $12271(4)$ & $5157(4)$ & $707(2)$ & $33(1)$ \\
\hline $\mathrm{C}(22)$ & $13401(4)$ & $4183(4)$ & $362(2)$ & $34(1)$ \\
\hline $\mathrm{C}(23)$ & $13398(4)$ & $3053(4)$ & $1094(3)$ & $33(1)$ \\
\hline $\mathrm{C}(24)$ & $12260(4)$ & $3357(4)$ & $1872(3)$ & $34(1)$ \\
\hline $\mathrm{C}(25)$ & $11756(4)$ & $6514(4)$ & $302(3)$ & $41(1)$ \\
\hline $\mathrm{C}(26)$ & $14448(4)$ & $4298(4)$ & $-611(2)$ & $38(1)$ \\
\hline $\mathrm{C}(27)$ & $15309(4)$ & $1443(4)$ & $1662(3)$ & $52(2)$ \\
\hline $\mathrm{C}(28)$ & $11681(4)$ & $2564(4)$ & $2807(3)$ & $39(1)$ \\
\hline $\mathrm{C}(29)$ & $11835(8)$ & $505(6)$ & $3849(6)$ & $135(3)$ \\
\hline & $12774(11)$ & $-343(12)$ & $4248(6)$ & $183(7)$ \\
\hline
\end{tabular}

Table 3. Bond lengths [A] and angles [deg] for compound 3.

\begin{tabular}{c|c}
$\mathrm{O}(1)-\mathrm{C}(5)$ & $1.228(5)$ \\
\hline $\mathrm{O}(2)-\mathrm{C}(3)$ & $1.355(5)$ \\
\hline $\mathrm{O}(2)-\mathrm{C}(7)$ & $1.430(7)$ \\
\hline $\mathrm{O}(3)-\mathrm{C}(8)$ & $1.223(5)$ \\
\hline $\mathrm{O}(4)-\mathrm{C}(8)$ & $1.310(6)$ \\
\hline $\mathrm{O}(4)-\mathrm{C}(9)$ & $1.468(5)$ \\
\hline $\mathrm{O}(5)-\mathrm{C}(13)$ & $1.369(6)$ \\
\hline $\mathrm{O}(5)-\mathrm{C}(17)$ & $1.437(6)$ \\
\hline $\mathrm{O}(6)-\mathrm{C}(15)$ & $1.222(5)$ \\
\hline $\mathrm{O}(7)-\mathrm{C}(18)$ & $1.221(5)$
\end{tabular}




\begin{tabular}{|c|c|}
\hline $\mathrm{O}(8)-\mathrm{C}(18)$ & $1.323(6)$ \\
\hline $\mathrm{O}(8)-\mathrm{C}(19)$ & $1.465(5)$ \\
\hline $\mathrm{O}(9)-\mathrm{C}(25)$ & $1.218(5)$ \\
\hline $\mathrm{O}(10)-\mathrm{C}(27)$ & $1.429(6)$ \\
\hline $\mathrm{O}(10)-\mathrm{C}(23)$ & $1.373(6)$ \\
\hline $\mathrm{O}(11)-\mathrm{C}(28)$ & $1.216(6)$ \\
\hline $\mathrm{O}(12)-\mathrm{C}(28)$ & $1.307(6)$ \\
\hline $\mathrm{O}(12)-\mathrm{C}(29)$ & $1.494(9)$ \\
\hline $\mathrm{N}(1)-\mathrm{C}(1)$ & $1.391(5)$ \\
\hline $\mathrm{N}(1)-\mathrm{C}(4)$ & $1.338(6)$ \\
\hline $\mathrm{N}(1)-\mathrm{H}(1)$ & 0.8808 \\
\hline $\mathrm{N}(2)-\mathrm{C}(14)$ & $1.355(5)$ \\
\hline $\mathrm{N}(2)-\mathrm{C}(11)$ & $1.382(4)$ \\
\hline $\mathrm{N}(2)-\mathrm{H}(2)$ & 0.8796 \\
\hline $\mathrm{N}(3)-\mathrm{C}(24)$ & $1.337(6)$ \\
\hline $\mathrm{N}(3)-\mathrm{C}(21)$ & $1.389(5)$ \\
\hline $\mathrm{N}(3)-\mathrm{H}(3)$ & 0.8798 \\
\hline $\mathrm{C}(1)-\mathrm{C}(5)$ & $1.414(7)$ \\
\hline $\mathrm{C}(1)-\mathrm{C}(2)$ & $1.394(6)$ \\
\hline $\mathrm{C}(2)-\mathrm{C}(6)$ & $1.520(6)$ \\
\hline$C(2)-C(3)$ & $1.394(6)$ \\
\hline$C(3)-C(4)$ & $1.416(6)$ \\
\hline$C(4)-C(8)$ & $1.473(6)$ \\
\hline $\mathrm{C}(9)-\mathrm{C}(10)$ & $1.479(6)$ \\
\hline $\mathrm{C}(5)-\mathrm{H}(5 \mathrm{~A})$ & 0.9492 \\
\hline $\mathrm{C}(6)-\mathrm{H}(6 \mathrm{C})$ & 0.9801 \\
\hline $\mathrm{C}(6)-\mathrm{H}(6 \mathrm{~B})$ & 0.9806 \\
\hline $\mathrm{C}(6)-\mathrm{H}(6 \mathrm{~A})$ & 0.9795 \\
\hline $\mathrm{C}(7)-\mathrm{H}(7 \mathrm{~B})$ & 0.9801 \\
\hline $\mathrm{C}(7)-\mathrm{H}(7 \mathrm{C})$ & 0.9799 \\
\hline $\mathrm{C}(7)-\mathrm{H}(7 \mathrm{~A})$ & 0.9806 \\
\hline $\mathrm{C}(9)-\mathrm{H}(9 \mathrm{~A})$ & 0.9900 \\
\hline $\mathrm{C}(9)-\mathrm{H}(9 \mathrm{~B})$ & 0.9902 \\
\hline $\mathrm{C}(10)-\mathrm{H}(10 \mathrm{~B})$ & 0.9802 \\
\hline $\mathrm{C}(10)-\mathrm{H}(10 \mathrm{~A})$ & 0.9807 \\
\hline $\mathrm{C}(10)-\mathrm{H}(10 \mathrm{C})$ & 0.9796 \\
\hline $\mathrm{C}(11)-\mathrm{C}(12)$ & $1.380(6)$ \\
\hline $\mathrm{C}(11)-\mathrm{C}(15)$ & $1.450(6)$ \\
\hline$C(12)-C(16)$ & $1.504(6)$ \\
\hline $\mathrm{C}(12)-\mathrm{C}(13)$ & $1.403(6)$ \\
\hline
\end{tabular}




\begin{tabular}{|c|c|}
\hline $\mathrm{C}(13)-\mathrm{C}(14)$ & $1.399(5)$ \\
\hline $\mathrm{C}(14)-\mathrm{C}(18)$ & $1.465(5)$ \\
\hline $\mathrm{C}(19)-\mathrm{C}(20)$ & $1.475(6)$ \\
\hline $\mathrm{C}(15)-\mathrm{H}(15 \mathrm{~A})$ & 0.9503 \\
\hline $\mathrm{C}(16)-\mathrm{H}(16 \mathrm{~A})$ & 0.9810 \\
\hline $\mathrm{C}(16)-\mathrm{H}(16 \mathrm{C})$ & 0.9793 \\
\hline $\mathrm{C}(16)-\mathrm{H}(16 \mathrm{~B})$ & 0.9801 \\
\hline $\mathrm{C}(17)-\mathrm{H}(17 \mathrm{~B})$ & 0.9794 \\
\hline $\mathrm{C}(17)-\mathrm{H}(17 \mathrm{C})$ & 0.9798 \\
\hline $\mathrm{C}(17)-\mathrm{H}(17 \mathrm{~A})$ & 0.9797 \\
\hline $\mathrm{C}(19)-\mathrm{H}(19 \mathrm{~B})$ & 0.9897 \\
\hline $\mathrm{C}(19)-\mathrm{H}(19 \mathrm{~A})$ & 0.9907 \\
\hline $\mathrm{C}(20)-\mathrm{H}(20 \mathrm{C})$ & 0.9804 \\
\hline $\mathrm{C}(20)-\mathrm{H}(20 \mathrm{~A})$ & 0.9808 \\
\hline $\mathrm{C}(20)-\mathrm{H}(20 \mathrm{~B})$ & 0.9791 \\
\hline $\mathrm{C}(21)-\mathrm{C}(25)$ & $1.439(6)$ \\
\hline $\mathrm{C}(21)-\mathrm{C}(22)$ & $1.387(6)$ \\
\hline $\mathrm{C}(22)-\mathrm{C}(23)$ & $1.395(6)$ \\
\hline $\mathrm{C}(22)-\mathrm{C}(26)$ & $1.504(5)$ \\
\hline $\mathrm{C}(23)-\mathrm{C}(24)$ & $1.394(6)$ \\
\hline $\mathrm{C}(24)-\mathrm{C}(28)$ & $1.482(6)$ \\
\hline $\mathrm{C}(29)-\mathrm{C}(30)$ & $1.239(15)$ \\
\hline $\mathrm{C}(25)-\mathrm{H}(25 \mathrm{~A})$ & 0.9495 \\
\hline $\mathrm{C}(26)-\mathrm{H}(26 \mathrm{~A})$ & 0.9800 \\
\hline $\mathrm{C}(26)-\mathrm{H}(26 \mathrm{~B})$ & 0.9795 \\
\hline $\mathrm{C}(26)-\mathrm{H}(26 \mathrm{C})$ & 0.9802 \\
\hline $\mathrm{C}(27)-\mathrm{H}(27 \mathrm{~A})$ & 0.9793 \\
\hline $\mathrm{C}(27)-\mathrm{H}(27 \mathrm{~B})$ & 0.9804 \\
\hline $\mathrm{C}(27)-\mathrm{H}(27 \mathrm{C})$ & 0.9806 \\
\hline $\mathrm{C}(29)-\mathrm{H}(29 \mathrm{~A})$ & 0.9903 \\
\hline $\mathrm{C}(29)-\mathrm{H}(29 \mathrm{~B})$ & 0.9901 \\
\hline $\mathrm{C}(30)-\mathrm{H}(30 \mathrm{~A})$ & 0.9803 \\
\hline $\mathrm{C}(30)-\mathrm{H}(30 \mathrm{~B})$ & 0.9793 \\
\hline $\mathrm{C}(30)-\mathrm{H}(30 \mathrm{C})$ & 0.9797 \\
\hline
\end{tabular}

\begin{tabular}{c|c}
$\mathrm{C}(3)-\mathrm{O}(2)-\mathrm{C}(7)$ & $112.4(4)$ \\
\hline $\mathrm{C}(8)-\mathrm{O}(4)-\mathrm{C}(9)$ & $116.2(3)$ \\
\hline $\mathrm{C}(13)-\mathrm{O}(5)-\mathrm{C}(17)$ & $112.7(4)$ \\
\hline $\mathrm{C}(18)-\mathrm{O}(8)-\mathrm{C}(19)$ & $116.8(3)$
\end{tabular}




\begin{tabular}{|c|c|}
\hline $\mathrm{C}(23)-\mathrm{O}(10)-\mathrm{C}(27)$ & $113.2(3)$ \\
\hline $\mathrm{C}(28)-\mathrm{O}(12)-\mathrm{C}(29)$ & $115.2(4)$ \\
\hline $\mathrm{C}(1)-\mathrm{N}(1)-\mathrm{C}(4)$ & $110.4(3)$ \\
\hline $\mathrm{C}(1)-\mathrm{N}(1)-\mathrm{H}(1)$ & 124.81 \\
\hline $\mathrm{C}(4)-\mathrm{N}(1)-\mathrm{H}(1)$ & 124.84 \\
\hline $\mathrm{C}(11)-\mathrm{N}(2)-\mathrm{C}(14)$ & 109.2(3) \\
\hline $\mathrm{C}(14)-\mathrm{N}(2)-\mathrm{H}(2)$ & 125.40 \\
\hline $\mathrm{C}(11)-\mathrm{N}(2)-\mathrm{H}(2)$ & 125.38 \\
\hline $\mathrm{C}(21)-\mathrm{N}(3)-\mathrm{C}(24)$ & 109.2(3) \\
\hline $\mathrm{C}(24)-\mathrm{N}(3)-\mathrm{H}(3)$ & 125.46 \\
\hline $\mathrm{C}(21)-\mathrm{N}(3)-\mathrm{H}(3)$ & 125.30 \\
\hline $\mathrm{N}(1)-\mathrm{C}(1)-\mathrm{C}(2)$ & $106.9(4)$ \\
\hline $\mathrm{N}(1)-\mathrm{C}(1)-\mathrm{C}(5)$ & $120.6(4)$ \\
\hline $\mathrm{C}(2)-\mathrm{C}(1)-\mathrm{C}(5)$ & $132.5(4)$ \\
\hline$C(3)-C(2)-C(6)$ & $125.7(4)$ \\
\hline $\mathrm{C}(1)-\mathrm{C}(2)-\mathrm{C}(6)$ & $126.5(4)$ \\
\hline $\mathrm{C}(1)-\mathrm{C}(2)-\mathrm{C}(3)$ & $107.8(4)$ \\
\hline $\mathrm{O}(2)-\mathrm{C}(3)-\mathrm{C}(4)$ & $127.3(4)$ \\
\hline $\mathrm{C}(2)-\mathrm{C}(3)-\mathrm{C}(4)$ & $107.3(4)$ \\
\hline $\mathrm{O}(2)-\mathrm{C}(3)-\mathrm{C}(2)$ & $125.4(4)$ \\
\hline $\mathrm{N}(1)-\mathrm{C}(4)-\mathrm{C}(8)$ & $119.9(4)$ \\
\hline $\mathrm{N}(1)-\mathrm{C}(4)-\mathrm{C}(3)$ & $107.7(4)$ \\
\hline$C(3)-C(4)-C(8)$ & $132.4(4)$ \\
\hline $\mathrm{O}(1)-\mathrm{C}(5)-\mathrm{C}(1)$ & $125.2(4)$ \\
\hline $\mathrm{O}(4)-\mathrm{C}(8)-\mathrm{C}(4)$ & $113.6(3)$ \\
\hline $\mathrm{O}(3)-\mathrm{C}(8)-\mathrm{C}(4)$ & $121.8(4)$ \\
\hline $\mathrm{O}(3)-\mathrm{C}(8)-\mathrm{O}(4)$ & $124.6(4)$ \\
\hline $\mathrm{O}(4)-\mathrm{C}(9)-\mathrm{C}(10)$ & $108.2(3)$ \\
\hline $\mathrm{O}(1)-\mathrm{C}(5)-\mathrm{H}(5 \mathrm{~A})$ & 117.41 \\
\hline $\mathrm{C}(1)-\mathrm{C}(5)-\mathrm{H}(5 \mathrm{~A})$ & 117.43 \\
\hline $\mathrm{C}(2)-\mathrm{C}(6)-\mathrm{H}(6 \mathrm{~B})$ & 109.43 \\
\hline $\mathrm{C}(2)-\mathrm{C}(6)-\mathrm{H}(6 \mathrm{~A})$ & 109.45 \\
\hline $\mathrm{H}(6 \mathrm{~B})-\mathrm{C}(6)-\mathrm{H}(6 \mathrm{C})$ & 109.48 \\
\hline $\mathrm{H}(6 \mathrm{~A})-\mathrm{C}(6)-\mathrm{H}(6 \mathrm{C})$ & 109.49 \\
\hline $\mathrm{H}(6 \mathrm{~A})-\mathrm{C}(6)-\mathrm{H}(6 \mathrm{~B})$ & 109.48 \\
\hline $\mathrm{C}(2)-\mathrm{C}(6)-\mathrm{H}(6 \mathrm{C})$ & 109.51 \\
\hline $\mathrm{H}(7 \mathrm{~A})-\mathrm{C}(7)-\mathrm{H}(7 \mathrm{C})$ & 109.52 \\
\hline $\mathrm{H}(7 \mathrm{~B})-\mathrm{C}(7)-\mathrm{H}(7 \mathrm{C})$ & 109.46 \\
\hline $\mathrm{H}(7 \mathrm{~A})-\mathrm{C}(7)-\mathrm{H}(7 \mathrm{~B})$ & 109.40 \\
\hline $\mathrm{O}(2)-\mathrm{C}(7)-\mathrm{H}(7 \mathrm{~A})$ & 109.46 \\
\hline
\end{tabular}




\begin{tabular}{c|c}
$\mathrm{O}(2)-\mathrm{C}(7)-\mathrm{H}(7 \mathrm{~B})$ & 109.45 \\
\hline $\mathrm{O}(2)-\mathrm{C}(7)-\mathrm{H}(7 \mathrm{C})$ & 109.53 \\
\hline $\mathrm{C}(10)-\mathrm{C}(9)-\mathrm{H}(9 \mathrm{~B})$ & 110.07 \\
\hline $\mathrm{C}(10)-\mathrm{C}(9)-\mathrm{H}(9 \mathrm{~A})$ & 109.94 \\
\hline $\mathrm{O}(4)-\mathrm{C}(9)-\mathrm{H}(9 \mathrm{~B})$ & 110.10 \\
\hline $\mathrm{H}(9 \mathrm{~A})-\mathrm{C}(9)-\mathrm{H}(9 \mathrm{~B})$ & 108.49 \\
\hline $\mathrm{O}(4)-\mathrm{C}(9)-\mathrm{H}(9 \mathrm{~A})$ & 110.04 \\
\hline $\mathrm{C}(9)-\mathrm{C}(10)-\mathrm{H}(10 \mathrm{~A})$ & 109.37 \\
\hline $\mathrm{H}(10 \mathrm{~A})-\mathrm{C}(10)-\mathrm{H}(10 \mathrm{~B})$ & 109.52 \\
\hline $\mathrm{H}(10 \mathrm{~A})-\mathrm{C}(10)-\mathrm{H}(10 \mathrm{C})$ & 109.46 \\
\hline $\mathrm{C}(9)-\mathrm{C}(10)-\mathrm{H}(10 \mathrm{C})$ & 109.46 \\
\hline $\mathrm{C}(9)-\mathrm{C}(10)-\mathrm{H}(10 \mathrm{~B})$ & 109.46 \\
\hline $\mathrm{H}(10 \mathrm{~B})-\mathrm{C}(10)-\mathrm{H}(10 \mathrm{C})$ & 109.55 \\
\hline $\mathrm{N}(2)-\mathrm{C}(11)-\mathrm{C}(15)$ & $119.3(4)$ \\
\hline $\mathrm{N}(2)-\mathrm{C}(11)-\mathrm{C}(12)$ & $108.7(4)$ \\
\hline $\mathrm{C}(12)-\mathrm{C}(11)-\mathrm{C}(15)$ & $132.0(3)$ \\
\hline $\mathrm{C}(13)-\mathrm{C}(12)-\mathrm{C}(16)$ & $125.8(4)$ \\
\hline $\mathrm{C}(11)-\mathrm{C}(12)-\mathrm{C}(16)$ & $127.7(4)$ \\
\hline $\mathrm{C}(11)-\mathrm{C}(12)-\mathrm{C}(13)$ & $106.4(4)$ \\
\hline $\mathrm{C}(12)-\mathrm{C}(13)-\mathrm{C}(14)$ & $108.3(4)$ \\
\hline $\mathrm{O}(5)-\mathrm{C}(13)-\mathrm{C}(14)$ & $126.7(4)$ \\
\hline $\mathrm{O}(5)-\mathrm{C}(13)-\mathrm{C}(12)$ & $125.1(4)$ \\
\hline $\mathrm{N}(2)-\mathrm{C}(14)-\mathrm{C}(13)$ & $107.4(3)$ \\
\hline $\mathrm{N}(2)-\mathrm{C}(14)-\mathrm{C}(18)$ & $119.1(3)$ \\
\hline $\mathrm{C}(13)-\mathrm{C}(14)-\mathrm{C}(18)$ & $133.5(3)$ \\
\hline $\mathrm{O}(6)-\mathrm{C}(15)-\mathrm{C}(11)$ & $124.2(4)$ \\
\hline $\mathrm{O}(8)-\mathrm{C}(18)-\mathrm{C}(14)$ & $112.6(3)$ \\
\hline $\mathrm{O}(7)-\mathrm{C}(18)-\mathrm{O}(8)$ & $124.4(4)$ \\
\hline $\mathrm{O}(7)-\mathrm{C}(18)-\mathrm{C}(14)$ & $123.0(4)$ \\
\hline $\mathrm{O}(8)-\mathrm{C}(19)-\mathrm{C}(20)$ & $107.8(3)$ \\
\hline $\mathrm{O}(6)-\mathrm{C}(15)-\mathrm{H}(15 \mathrm{~A})$ & 117.87 \\
\hline $\mathrm{C}(11)-\mathrm{C}(15)-\mathrm{H}(15 \mathrm{~A})$ & 117.88 \\
\hline $\mathrm{H}(16 \mathrm{~A})-\mathrm{C}(16)-\mathrm{H}(16 \mathrm{C})$ & 109.48 \\
\hline $\mathrm{C}(12)-\mathrm{C}(16)-\mathrm{H}(16 \mathrm{~A})$ & 109.48 \\
\hline $\mathrm{H}(17 \mathrm{C})-\mathrm{C}(16)-\mathrm{H}(16 \mathrm{~B})$ & 109.40 \\
\hline $\mathrm{H}(16 \mathrm{~B})$ & 109.52 \\
\hline $\mathrm{H}(16 \mathrm{C})$ & 109.52 \\
\hline $\mathrm{H}(16) .46$ \\
\hline
\end{tabular}




\begin{tabular}{|c|c|}
\hline $\mathrm{O}(5)-\mathrm{C}(17)-\mathrm{H}(17 \mathrm{~A})$ & 109.47 \\
\hline $\mathrm{H}(17 \mathrm{~A})-\mathrm{C}(17)-\mathrm{H}(17 \mathrm{~B})$ & 109.47 \\
\hline $\mathrm{H}(17 \mathrm{~A})-\mathrm{C}(17)-\mathrm{H}(17 \mathrm{C})$ & 109.42 \\
\hline $\mathrm{O}(5)-\mathrm{C}(17)-\mathrm{H}(17 \mathrm{C})$ & 109.51 \\
\hline $\mathrm{C}(20)-\mathrm{C}(19)-\mathrm{H}(19 \mathrm{~A})$ & 110.08 \\
\hline $\mathrm{C}(20)-\mathrm{C}(19)-\mathrm{H}(19 \mathrm{~B})$ & 110.17 \\
\hline $\mathrm{O}(8)-\mathrm{C}(19)-\mathrm{H}(19 \mathrm{~B})$ & 110.21 \\
\hline $\mathrm{H}(19 \mathrm{~A})-\mathrm{C}(19)-\mathrm{H}(19 \mathrm{~B})$ & 108.45 \\
\hline $\mathrm{O}(8)-\mathrm{C}(19)-\mathrm{H}(19 \mathrm{~A})$ & 110.19 \\
\hline $\mathrm{C}(19)-\mathrm{C}(20)-\mathrm{H}(20 \mathrm{C})$ & 109.48 \\
\hline $\mathrm{H}(20 \mathrm{~A})-\mathrm{C}(20)-\mathrm{H}(20 \mathrm{~B})$ & 109.47 \\
\hline $\mathrm{C}(19)-\mathrm{C}(20)-\mathrm{H}(20 \mathrm{~A})$ & 109.39 \\
\hline $\mathrm{C}(19)-\mathrm{C}(20)-\mathrm{H}(20 \mathrm{~B})$ & 109.52 \\
\hline $\mathrm{H}(20 \mathrm{~B})-\mathrm{C}(20)-\mathrm{H}(20 \mathrm{C})$ & 109.48 \\
\hline $\mathrm{H}(20 \mathrm{~A})-\mathrm{C}(20)-\mathrm{H}(20 \mathrm{C})$ & 109.48 \\
\hline $\mathrm{N}(3)-\mathrm{C}(21)-\mathrm{C}(25)$ & $119.6(4)$ \\
\hline $\mathrm{N}(3)-\mathrm{C}(21)-\mathrm{C}(22)$ & $107.6(3)$ \\
\hline $\mathrm{C}(22)-\mathrm{C}(21)-\mathrm{C}(25)$ & $132.8(3)$ \\
\hline $\mathrm{C}(23)-\mathrm{C}(22)-\mathrm{C}(26)$ & $125.6(4)$ \\
\hline$C(21)-C(22)-C(26)$ & $127.3(3)$ \\
\hline $\mathrm{C}(21)-\mathrm{C}(22)-\mathrm{C}(23)$ & 107.1(3) \\
\hline $\mathrm{O}(10)-\mathrm{C}(23)-\mathrm{C}(22)$ & $125.0(4)$ \\
\hline $\mathrm{O}(10)-\mathrm{C}(23)-\mathrm{C}(24)$ & $127.4(4)$ \\
\hline $\mathrm{C}(22)-\mathrm{C}(23)-\mathrm{C}(24)$ & $107.6(4)$ \\
\hline $\mathrm{N}(3)-\mathrm{C}(24)-\mathrm{C}(23)$ & $108.5(4)$ \\
\hline $\mathrm{C}(23)-\mathrm{C}(24)-\mathrm{C}(28)$ & $132.5(4)$ \\
\hline $\mathrm{N}(3)-\mathrm{C}(24)-\mathrm{C}(28)$ & $118.9(4)$ \\
\hline $\mathrm{O}(9)-\mathrm{C}(25)-\mathrm{C}(21)$ & $125.4(4)$ \\
\hline $\mathrm{O}(11)-\mathrm{C}(28)-\mathrm{O}(12)$ & $124.7(4)$ \\
\hline $\mathrm{O}(11)-\mathrm{C}(28)-\mathrm{C}(24)$ & $122.1(4)$ \\
\hline $\mathrm{O}(12)-\mathrm{C}(28)-\mathrm{C}(24)$ & $113.2(4)$ \\
\hline $\mathrm{O}(12)-\mathrm{C}(29)-\mathrm{C}(30)$ & $108.0(9)$ \\
\hline $\mathrm{O}(9)-\mathrm{C}(25)-\mathrm{H}(25 \mathrm{~A})$ & 117.30 \\
\hline $\mathrm{C}(21)-\mathrm{C}(25)-\mathrm{H}(25 \mathrm{~A})$ & 117.26 \\
\hline $\mathrm{C}(22)-\mathrm{C}(26)-\mathrm{H}(26 \mathrm{~A})$ & 109.46 \\
\hline $\mathrm{C}(22)-\mathrm{C}(26)-\mathrm{H}(26 \mathrm{~B})$ & 109.51 \\
\hline $\mathrm{C}(22)-\mathrm{C}(26)-\mathrm{H}(26 \mathrm{C})$ & 109.48 \\
\hline $\mathrm{H}(26 \mathrm{~A})-\mathrm{C}(26)-\mathrm{H}(26 \mathrm{~B})$ & 109.48 \\
\hline $\mathrm{H}(26 \mathrm{~A})-\mathrm{C}(26)-\mathrm{H}(26 \mathrm{C})$ & 109.40 \\
\hline $\mathrm{H}(26 \mathrm{~B})-\mathrm{C}(26)-\mathrm{H}(26 \mathrm{C})$ & 109.51 \\
\hline
\end{tabular}




\begin{tabular}{c|c}
$\mathrm{O}(10)-\mathrm{C}(27)-\mathrm{H}(27 \mathrm{~A})$ & 109.44 \\
\hline $\mathrm{O}(10)-\mathrm{C}(27)-\mathrm{H}(27 \mathrm{~B})$ & 109.46 \\
\hline $\mathrm{O}(10)-\mathrm{C}(27)-\mathrm{H}(27 \mathrm{C})$ & 109.44 \\
\hline $\mathrm{H}(27 \mathrm{~A})-\mathrm{C}(27)-\mathrm{H}(27 \mathrm{~B})$ & 109.50 \\
\hline $\mathrm{H}(27 \mathrm{~A})-\mathrm{C}(27)-\mathrm{H}(27 \mathrm{C})$ & 109.50 \\
\hline $\mathrm{H}(27 \mathrm{~B})-\mathrm{C}(27)-\mathrm{H}(27 \mathrm{C})$ & 109.50 \\
\hline $\mathrm{O}(12)-\mathrm{C}(29)-\mathrm{H}(29 \mathrm{~A})$ & 110.09 \\
\hline $\mathrm{O}(12)-\mathrm{C}(29)-\mathrm{H}(29 \mathrm{~B})$ & 110.15 \\
\hline $\mathrm{C}(30)-\mathrm{C}(29)-\mathrm{H}(29 \mathrm{~A})$ & 110.06 \\
\hline $\mathrm{C}(30)-\mathrm{C}(29)-\mathrm{H}(29 \mathrm{~B})$ & 110.10 \\
\hline $\mathrm{H}(29 \mathrm{~A})-\mathrm{C}(29)-\mathrm{H}(29 \mathrm{~B})$ & 108.44 \\
\hline $\mathrm{C}(29)-\mathrm{C}(30)-\mathrm{H}(30 \mathrm{~A})$ & 109.47 \\
\hline $\mathrm{C}(29)-\mathrm{C}(30)-\mathrm{H}(30 \mathrm{~B})$ & 109.49 \\
\hline $\mathrm{C}(29)-\mathrm{C}(30)-\mathrm{H}(30 \mathrm{C})$ & 109.47 \\
\hline $\mathrm{H}(30 \mathrm{~A})-\mathrm{C}(30)-\mathrm{H}(30 \mathrm{~B})$ & 109.44 \\
\hline $\mathrm{H}(30 \mathrm{~A})-\mathrm{C}(30)-\mathrm{H}(30 \mathrm{C})$ & 109.43 \\
\hline $\mathrm{H}(30 \mathrm{~B})-\mathrm{C}(30)-\mathrm{H}(30 \mathrm{C})$ & 109.54 \\
\hline
\end{tabular}

Table 4. Anisotropic displacement parameters $\left(\mathrm{A}^{\wedge} 2 \times 10^{\wedge} 3\right)$ for compound 3 . The anisotropic displacement factor exponent takes the form: $-2 \mathrm{pi}^{\wedge} 2\left[\mathrm{~h}^{\wedge} 2 \mathrm{a}^{* \wedge} 2 \mathrm{U} 11+\ldots+2 \mathrm{~h} \mathrm{k} \mathrm{a}^{*} \mathrm{~b}^{*} \mathrm{U} 12\right.$ ]

\begin{tabular}{c|c|c|c|c|c|c} 
& $\mathrm{U} 11$ & $\mathrm{U} 22$ & $\mathrm{U} 33$ & $\mathrm{U} 23$ & $\mathrm{U} 13$ & $\mathrm{U} 12$ \\
\hline $\mathrm{O}(1)$ & $106(3)$ & $77(2)$ & $40(2)$ & $8(2)$ & $-9(2)$ & $-59(2)$ \\
\hline $\mathrm{O}(2)$ & $64(2)$ & $65(2)$ & $37(1)$ & $-27(1)$ & $5(1)$ & $-31(2)$ \\
\hline $\mathrm{O}(3)$ & $45(2)$ & $46(2)$ & $27(1)$ & $-9(1)$ & $1(1)$ & $-17(1)$ \\
\hline $\mathrm{O}(4)$ & $49(2)$ & $44(2)$ & $27(1)$ & $-8(1)$ & $2(1)$ & $-21(1)$ \\
\hline $\mathrm{N}(1)$ & $45(2)$ & $45(2)$ & $24(1)$ & $-4(1)$ & $-2(1)$ & $-23(2)$ \\
\hline $\mathrm{C}(1)$ & $48(3)$ & $57(3)$ & $26(2)$ & $-1(2)$ & $-9(2)$ & $-28(2)$ \\
\hline $\mathrm{C}(2)$ & $52(3)$ & $69(3)$ & $26(2)$ & $-12(2)$ & $-2(2)$ & $-41(2)$ \\
\hline $\mathrm{C}(3)$ & $42(2)$ & $56(3)$ & $28(2)$ & $-14(2)$ & $-1(2)$ & $-25(2)$ \\
\hline $\mathrm{C}(4)$ & $34(2)$ & $49(3)$ & $29(2)$ & $-9(2)$ & $-4(2)$ & $-21(2)$ \\
\hline $\mathrm{C}(5)$ & $72(3)$ & $60(3)$ & $36(2)$ & $2(2)$ & $-12(2)$ & $-38(3)$ \\
\hline $\mathrm{C}(6)$ & $90(4)$ & $108(4)$ & $28(2)$ & $-25(2)$ & $12(2)$ & $-76(4)$ \\
\hline $\mathrm{C}(7)$ & $71(4)$ & $63(3)$ & $72(3)$ & $-27(3)$ & $1(3)$ & $-23(3)$ \\
\hline $\mathrm{C}(8)$ & $30(2)$ & $42(3)$ & $32(2)$ & $-12(2)$ & $-4(2)$ & $-12(2)$ \\
\hline $\mathrm{C}(9)$ & $56(3)$ & $47(3)$ & $32(2)$ & $0(2)$ & $-2(2)$ & $-27(2)$ \\
\hline $\mathrm{C}(10)$ & $69(3)$ & $44(3)$ & $47(2)$ & $-6(2)$ & $-6(2)$ & $-20(3)$ \\
\hline $\mathrm{O}(5)$ & $44(2)$ & $43(2)$ & $37(1)$ & $-17(1)$ & $-1(1)$ & $-18(1)$ \\
\hline & & & & & & \\
\hline & & & & & \\
\hline & & & & \\
\hline
\end{tabular}




\begin{tabular}{c|c|c|c|c|c|c}
$\mathrm{O}(6)$ & $93(3)$ & $59(2)$ & $46(2)$ & $5(2)$ & $0(2)$ & $-42(2)$ \\
\hline $\mathrm{O}(7)$ & $43(2)$ & $42(2)$ & $31(1)$ & $-10(1)$ & $1(1)$ & $-17(1)$ \\
\hline $\mathrm{O}(8)$ & $50(2)$ & $39(2)$ & $29(1)$ & $-3(1)$ & $4(1)$ & $-20(2)$ \\
\hline $\mathrm{N}(2)$ & $39(2)$ & $43(2)$ & $25(1)$ & $-8(1)$ & $-1(1)$ & $-18(2)$ \\
\hline $\mathrm{C}(11)$ & $42(2)$ & $45(2)$ & $24(2)$ & $-4(2)$ & $-4(2)$ & $-24(2)$ \\
\hline $\mathrm{C}(12)$ & $37(2)$ & $51(3)$ & $26(2)$ & $-13(2)$ & $-4(2)$ & $-21(2)$ \\
\hline $\mathrm{C}(13)$ & $33(2)$ & $39(2)$ & $30(2)$ & $-12(2)$ & $-5(2)$ & $-15(2)$ \\
\hline $\mathrm{C}(14)$ & $33(2)$ & $30(2)$ & $27(2)$ & $-10(2)$ & $-5(2)$ & $-9(2)$ \\
\hline $\mathrm{C}(15)$ & $58(3)$ & $51(3)$ & $37(2)$ & $-6(2)$ & $-7(2)$ & $-27(2)$ \\
\hline $\mathrm{C}(16)$ & $45(2)$ & $66(3)$ & $28(2)$ & $-11(2)$ & $2(2)$ & $-32(2)$ \\
\hline $\mathrm{C}(17)$ & $44(3)$ & $45(3)$ & $64(3)$ & $-14(2)$ & $-10(2)$ & $-10(2)$ \\
\hline $\mathrm{C}(18)$ & $29(2)$ & $36(2)$ & $29(2)$ & $-8(2)$ & $-7(2)$ & $-11(2)$ \\
\hline $\mathrm{C}(19)$ & $65(3)$ & $53(3)$ & $33(2)$ & $-1(2)$ & $6(2)$ & $-33(3)$ \\
\hline $\mathrm{C}(20)$ & $72(3)$ & $46(3)$ & $45(2)$ & $1(2)$ & $-2(2)$ & $-31(3)$ \\
\hline $\mathrm{O}(9)$ & $72(2)$ & $57(2)$ & $38(1)$ & $7(1)$ & $-8(1)$ & $-40(2)$ \\
\hline $\mathrm{O}(10)$ & $41(2)$ & $44(2)$ & $38(1)$ & $-13(1)$ & $-2(1)$ & $-15(1)$ \\
\hline $\mathrm{O}(11)$ & $52(2)$ & $46(2)$ & $41(2)$ & $-6(1)$ & $8(1)$ & $-24(2)$ \\
\hline $\mathrm{O}(12)$ & $69(2)$ & $36(2)$ & $55(2)$ & $-2(1)$ & $21(2)$ & $-22(2)$ \\
\hline $\mathrm{N}(3)$ & $33(2)$ & $38(2)$ & $26(1)$ & $-6(1)$ & $0(1)$ & $-17(2)$ \\
\hline $\mathrm{C}(21)$ & $38(2)$ & $44(2)$ & $23(2)$ & $-7(2)$ & $-2(2)$ & $-24(2)$ \\
\hline $\mathrm{C}(22)$ & $40(2)$ & $46(2)$ & $24(2)$ & $-10(2)$ & $-5(2)$ & $-22(2)$ \\
\hline $\mathrm{C}(23)$ & $37(2)$ & $37(2)$ & $27(2)$ & $-12(2)$ & $-2(2)$ & $-16(2)$ \\
\hline $\mathrm{C}(24)$ & $36(2)$ & $35(2)$ & $32(2)$ & $-8(2)$ & $-6(2)$ & $-16(2)$ \\
\hline $\mathrm{C}(25)$ & $47(2)$ & $47(3)$ & $35(2)$ & $-4(2)$ & $-11(2)$ & $-25(2)$ \\
\hline $\mathrm{C}(26)$ & $44(2)$ & $53(3)$ & $26(2)$ & $-13(2)$ & $3(2)$ & $-31(2)$ \\
\hline $\mathrm{C}(27)$ & $44(3)$ & $53(3)$ & $47(2)$ & $-7(2)$ & $-7(2)$ & $-13(2)$ \\
\hline $\mathrm{C}(28)$ & $41(2)$ & $38(3)$ & $35(2)$ & $-10(2)$ & $3(2)$ & $-19(2)$ \\
\hline $\mathrm{C}(29)$ & $132(7)$ & $36(3)$ & $108(5)$ & $21(3)$ & $35(5)$ & $9(4)$ \\
\hline $\mathrm{C}(30)$ & $230(12)$ & $273(15)$ & $96(5)$ & $64(7)$ & $-69(6)$ & $-192(12)$ \\
& & & &
\end{tabular}

Table 5. Hydrogen coordinates ( $\left.\mathrm{x} 10^{\wedge} 4\right)$ and isotropic displacement parameters $\left(\mathrm{A}^{\wedge} 2 \mathrm{x}\right.$ $10^{\wedge} 3$ ) for compound 3 .

\begin{tabular}{c|c|c|c|c} 
& $\mathrm{x}$ & $\mathrm{y}$ & $\mathrm{z}$ & $\mathrm{U}(\mathrm{eq})$ \\
\hline & & & & \\
\hline $\mathrm{H}(1)$ & 9415 & 4352 & 4126 & 46 \\
\hline $\mathrm{H}(5 \mathrm{~A})$ & 9208 & 2369 & 5364 & 65 \\
\hline $\mathrm{H}(6 \mathrm{~A})$ & 5924 & 4611 & 7194 & 77 \\
\hline $\mathrm{H}(6 \mathrm{~B})$ & 7127 & 4384 & 7677 & 77 \\
\hline $\mathrm{H}(6 \mathrm{C})$ & 6070 & 5794 & 7352 & 77
\end{tabular}




\begin{tabular}{|c|c|c|c|c|}
\hline $\mathrm{H}(7 \mathrm{~A})$ & 5764 & 8257 & 4599 & 85 \\
\hline $\mathrm{H}(7 \mathrm{~B})$ & 5020 & 7623 & 5688 & 85 \\
\hline $\mathrm{H}(7 \mathrm{C})$ & 5167 & 8938 & 5539 & 85 \\
\hline $\mathrm{H}(9 \mathrm{~A})$ & 9659 & 8605 & 2291 & 56 \\
\hline $\mathrm{H}(9 \mathrm{~B})$ & 8231 & 8966 & 2048 & 56 \\
\hline $\mathrm{H}(10 \mathrm{~A})$ & 7244 & 10481 & 3033 & 67 \\
\hline $\mathrm{H}(10 \mathrm{~B})$ & 8662 & 10115 & 3287 & 67 \\
\hline $\mathrm{H}(10 \mathrm{C})$ & 8433 & 10779 & 2129 & 67 \\
\hline $\mathrm{H}(2)$ & 9119 & 4724 & 1062 & 44 \\
\hline $\mathrm{H}(15 \mathrm{~A})$ & 8804 & 2826 & 2386 & 58 \\
\hline $\mathrm{H}(16 \mathrm{~A})$ & 5203 & 5285 & 3817 & 53 \\
\hline $\mathrm{H}(16 \mathrm{~B})$ & 6247 & 5226 & 4384 & 53 \\
\hline $\mathrm{H}(16 \mathrm{C})$ & 5223 & 6573 & 3890 & 53 \\
\hline $\mathrm{H}(17 \mathrm{~A})$ & 5291 & 8631 & 934 & 64 \\
\hline $\mathrm{H}(17 \mathrm{~B})$ & 4409 & 8105 & 1993 & 64 \\
\hline $\mathrm{H}(17 \mathrm{C})$ & 4450 & 9487 & 1757 & 64 \\
\hline $\mathrm{H}(19 \mathrm{~A})$ & 9447 & 8885 & -964 & 64 \\
\hline $\mathrm{H}(19 \mathrm{~B})$ & 8128 & 9249 & -1371 & 64 \\
\hline $\mathrm{H}(20 \mathrm{~A})$ & 6972 & 10858 & -471 & 68 \\
\hline $\mathrm{H}(20 \mathrm{~B})$ & 8291 & 10499 & -74 & 68 \\
\hline $\mathrm{H}(20 \mathrm{C})$ & 8264 & 11082 & -1277 & 68 \\
\hline $\mathrm{H}(3)$ & 10811 & 5028 & 1990 & 40 \\
\hline $\mathrm{H}(25 \mathrm{~A})$ & 10965 & 6999 & 702 & 50 \\
\hline $\mathrm{H}(26 \mathrm{~A})$ & 14029 & 4661 & -1212 & 45 \\
\hline $\mathrm{H}(26 \mathrm{~B})$ & 14863 & 4854 & -602 & 45 \\
\hline $\mathrm{H}(26 \mathrm{C})$ & 15134 & 3453 & -654 & 45 \\
\hline $\mathrm{H}(27 \mathrm{~A})$ & 15773 & 2038 & 1439 & 63 \\
\hline $\mathrm{H}(27 \mathrm{~B})$ & 14844 & 1405 & 2387 & 63 \\
\hline $\mathrm{H}(27 \mathrm{C})$ & 15962 & 594 & 1590 & 63 \\
\hline $\mathrm{H}(29 \mathrm{~A})$ & 11174 & 1005 & 4353 & 162 \\
\hline $\mathrm{H}(29 \mathrm{~B})$ & 11379 & 124 & 3634 & 162 \\
\hline $\mathrm{H}(30 \mathrm{~A})$ & 13412 & -845 & 3749 & 221 \\
\hline $\mathrm{H}(30 \mathrm{~B})$ & 12427 & -899 & 4861 & 221 \\
\hline $\mathrm{H}(30 \mathrm{C})$ & 13227 & 44 & 4448 & 221 \\
\hline
\end{tabular}

Table 6. Torsion angles [deg] for compound $\mathbf{3}$.

\begin{tabular}{c|c}
$\mathrm{C}(7)-\mathrm{O}(2)-\mathrm{C}(3)-\mathrm{C}(4)$ & $87.1(6)$ \\
\hline $\mathrm{C}(7)-\mathrm{O}(2)-\mathrm{C}(3)-\mathrm{C}(2)$ & $-94.7(5)$ \\
\hline $\mathrm{C}(8)-\mathrm{O}(4)-\mathrm{C}(9)-\mathrm{C}(10)$ & $-171.9(5)$
\end{tabular}




\begin{tabular}{|c|c|}
\hline $\mathrm{C}(9)-\mathrm{O}(4)-\mathrm{C}(8)-\mathrm{C}(4)$ & $-175.7(4)$ \\
\hline $\mathrm{C}(9)-\mathrm{O}(4)-\mathrm{C}(8)-\mathrm{O}(3)$ & $4.1(7)$ \\
\hline $\mathrm{C}(17)-\mathrm{O}(5)-\mathrm{C}(13)-\mathrm{C}(14)$ & $84.9(6)$ \\
\hline $\mathrm{C}(17)-\mathrm{O}(5)-\mathrm{C}(13)-\mathrm{C}(12)$ & $-95.1(5)$ \\
\hline $\mathrm{C}(19)-\mathrm{O}(8)-\mathrm{C}(18)-\mathrm{C}(14)$ & $-178.3(4)$ \\
\hline $\mathrm{C}(18)-\mathrm{O}(8)-\mathrm{C}(19)-\mathrm{C}(20)$ & $-168.0(4)$ \\
\hline $\mathrm{C}(19)-\mathrm{O}(8)-\mathrm{C}(18)-\mathrm{O}(7)$ & $2.7(7)$ \\
\hline $\mathrm{C}(27)-\mathrm{O}(10)-\mathrm{C}(23)-\mathrm{C}(24)$ & $-79.8(6)$ \\
\hline $\mathrm{C}(27)-\mathrm{O}(10)-\mathrm{C}(23)-\mathrm{C}(22)$ & $100.4(5)$ \\
\hline $\mathrm{C}(29)-\mathrm{O}(12)-\mathrm{C}(28)-\mathrm{O}(11)$ & $1.9(8)$ \\
\hline $\mathrm{C}(29)-\mathrm{O}(12)-\mathrm{C}(28)-\mathrm{C}(24)$ & $-178.5(5)$ \\
\hline $\mathrm{C}(28)-\mathrm{O}(12)-\mathrm{C}(29)-\mathrm{C}(30)$ & $-139.6(7)$ \\
\hline $\mathrm{C}(1)-\mathrm{N}(1)-\mathrm{C}(4)-\mathrm{C}(8)$ & $-178.5(4)$ \\
\hline $\mathrm{C}(4)-\mathrm{N}(1)-\mathrm{C}(1)-\mathrm{C}(5)$ & $-179.3(5)$ \\
\hline $\mathrm{C}(1)-\mathrm{N}(1)-\mathrm{C}(4)-\mathrm{C}(3)$ & $0.2(5)$ \\
\hline $\mathrm{C}(4)-\mathrm{N}(1)-\mathrm{C}(1)-\mathrm{C}(2)$ & $-0.1(6)$ \\
\hline $\mathrm{C}(11)-\mathrm{N}(2)-\mathrm{C}(14)-\mathrm{C}(13)$ & $1.8(5)$ \\
\hline $\mathrm{C}(14)-\mathrm{N}(2)-\mathrm{C}(11)-\mathrm{C}(12)$ & $-2.2(5)$ \\
\hline $\mathrm{C}(11)-\mathrm{N}(2)-\mathrm{C}(14)-\mathrm{C}(18)$ & $179.9(4)$ \\
\hline $\mathrm{C}(14)-\mathrm{N}(2)-\mathrm{C}(11)-\mathrm{C}(15)$ & $178.6(4)$ \\
\hline $\mathrm{C}(21)-\mathrm{N}(3)-\mathrm{C}(24)-\mathrm{C}(23)$ & $-1.8(5)$ \\
\hline $\mathrm{C}(24)-\mathrm{N}(3)-\mathrm{C}(21)-\mathrm{C}(25)$ & $-177.8(4)$ \\
\hline $\mathrm{C}(21)-\mathrm{N}(3)-\mathrm{C}(24)-\mathrm{C}(28)$ & $-177.5(4)$ \\
\hline $\mathrm{C}(24)-\mathrm{N}(3)-\mathrm{C}(21)-\mathrm{C}(22)$ & $2.0(5)$ \\
\hline $\mathrm{N}(1)-\mathrm{C}(1)-\mathrm{C}(2)-\mathrm{C}(3)$ & $0.0(6)$ \\
\hline $\mathrm{N}(1)-\mathrm{C}(1)-\mathrm{C}(2)-\mathrm{C}(6)$ & $178.1(5)$ \\
\hline $\mathrm{C}(2)-\mathrm{C}(1)-\mathrm{C}(5)-\mathrm{O}(1)$ & $-1.0(11)$ \\
\hline $\mathrm{C}(5)-\mathrm{C}(1)-\mathrm{C}(2)-\mathrm{C}(6)$ & $-2.9(10)$ \\
\hline $\mathrm{C}(5)-\mathrm{C}(1)-\mathrm{C}(2)-\mathrm{C}(3)$ & $179.0(6)$ \\
\hline $\mathrm{N}(1)-\mathrm{C}(1)-\mathrm{C}(5)-\mathrm{O}(1)$ & $177.9(5)$ \\
\hline $\mathrm{C}(1)-\mathrm{C}(2)-\mathrm{C}(3)-\mathrm{C}(4)$ & $0.2(6)$ \\
\hline $\mathrm{C}(1)-\mathrm{C}(2)-\mathrm{C}(3)-\mathrm{O}(2)$ & $-178.4(5)$ \\
\hline $\mathrm{C}(6)-\mathrm{C}(2)-\mathrm{C}(3)-\mathrm{C}(4)$ & $-178.0(5)$ \\
\hline $\mathrm{C}(6)-\mathrm{C}(2)-\mathrm{C}(3)-\mathrm{O}(2)$ & $3.5(8)$ \\
\hline $\mathrm{O}(2)-\mathrm{C}(3)-\mathrm{C}(4)-\mathrm{C}(8)$ & $-3.2(9)$ \\
\hline $\mathrm{O}(2)-\mathrm{C}(3)-\mathrm{C}(4)-\mathrm{N}(1)$ & $178.3(5)$ \\
\hline $\mathrm{C}(2)-\mathrm{C}(3)-\mathrm{C}(4)-\mathrm{N}(1)$ & $-0.2(6)$ \\
\hline $\mathrm{C}(2)-\mathrm{C}(3)-\mathrm{C}(4)-\mathrm{C}(8)$ & $178.3(5)$ \\
\hline $\mathrm{C}(3)-\mathrm{C}(4)-\mathrm{C}(8)-\mathrm{O}(3)$ & $-176.7(5)$ \\
\hline $\mathrm{C}(3)-\mathrm{C}(4)-\mathrm{C}(8)-\mathrm{O}(4)$ & $3.1(8)$ \\
\hline
\end{tabular}




\begin{tabular}{|c|c|}
\hline $\mathrm{N}(1)-\mathrm{C}(4)-\mathrm{C}(8)-\mathrm{O}(3)$ & $1.7(8)$ \\
\hline $\mathrm{N}(1)-\mathrm{C}(4)-\mathrm{C}(8)-\mathrm{O}(4)$ & $-178.5(4)$ \\
\hline $\mathrm{N}(2)-\mathrm{C}(11)-\mathrm{C}(12)-\mathrm{C}(16)$ & $179.5(5)$ \\
\hline $\mathrm{N}(2)-\mathrm{C}(11)-\mathrm{C}(12)-\mathrm{C}(13)$ & $1.6(5)$ \\
\hline $\mathrm{N}(2)-\mathrm{C}(11)-\mathrm{C}(15)-\mathrm{O}(6)$ & $178.7(5)$ \\
\hline $\mathrm{C}(15)-\mathrm{C}(11)-\mathrm{C}(12)-\mathrm{C}(16)$ & $-1.4(9)$ \\
\hline $\mathrm{C}(15)-\mathrm{C}(11)-\mathrm{C}(12)-\mathrm{C}(13)$ & $-179.4(5)$ \\
\hline $\mathrm{C}(12)-\mathrm{C}(11)-\mathrm{C}(15)-\mathrm{O}(6)$ & $-0.3(10)$ \\
\hline $\mathrm{C}(16)-\mathrm{C}(12)-\mathrm{C}(13)-\mathrm{C}(14)$ & $-178.4(5)$ \\
\hline $\mathrm{C}(11)-\mathrm{C}(12)-\mathrm{C}(13)-\mathrm{C}(14)$ & $-0.4(6)$ \\
\hline $\mathrm{C}(16)-\mathrm{C}(12)-\mathrm{C}(13)-\mathrm{O}(5)$ & $1.5(8)$ \\
\hline $\mathrm{C}(11)-\mathrm{C}(12)-\mathrm{C}(13)-\mathrm{O}(5)$ & $179.5(5)$ \\
\hline $\mathrm{O}(5)-\mathrm{C}(13)-\mathrm{C}(14)-\mathrm{N}(2)$ & $179.2(4)$ \\
\hline $\mathrm{O}(5)-\mathrm{C}(13)-\mathrm{C}(14)-\mathrm{C}(18)$ & $1.5(9)$ \\
\hline $\mathrm{C}(12)-\mathrm{C}(13)-\mathrm{C}(14)-\mathrm{N}(2)$ & $-0.9(5)$ \\
\hline $\mathrm{C}(12)-\mathrm{C}(13)-\mathrm{C}(14)-\mathrm{C}(18)$ & $-178.6(5)$ \\
\hline $\mathrm{N}(2)-\mathrm{C}(14)-\mathrm{C}(18)-\mathrm{O}(7)$ & $4.1(7)$ \\
\hline $\mathrm{C}(13)-\mathrm{C}(14)-\mathrm{C}(18)-\mathrm{O}(8)$ & $2.5(8)$ \\
\hline $\mathrm{C}(13)-\mathrm{C}(14)-\mathrm{C}(18)-\mathrm{O}(7)$ & $-178.4(5)$ \\
\hline $\mathrm{N}(2)-\mathrm{C}(14)-\mathrm{C}(18)-\mathrm{O}(8)$ & $-175.0(4)$ \\
\hline $\mathrm{C}(22)-\mathrm{C}(21)-\mathrm{C}(25)-\mathrm{O}(9)$ & $3.0(9)$ \\
\hline $\mathrm{N}(3)-\mathrm{C}(21)-\mathrm{C}(22)-\mathrm{C}(23)$ & $-1.4(5)$ \\
\hline $\mathrm{C}(25)-\mathrm{C}(21)-\mathrm{C}(22)-\mathrm{C}(26)$ & $-1.4(9)$ \\
\hline $\mathrm{N}(3)-\mathrm{C}(21)-\mathrm{C}(25)-\mathrm{O}(9)$ & $-177.3(5)$ \\
\hline$C(25)-C(21)-C(22)-C(23)$ & $178.4(5)$ \\
\hline $\mathrm{N}(3)-\mathrm{C}(21)-\mathrm{C}(22)-\mathrm{C}(26)$ & $178.8(4)$ \\
\hline $\mathrm{C}(26)-\mathrm{C}(22)-\mathrm{C}(23)-\mathrm{C}(24)$ & $-179.9(4)$ \\
\hline $\mathrm{C}(26)-\mathrm{C}(22)-\mathrm{C}(23)-\mathrm{O}(10)$ & $-0.1(8)$ \\
\hline $\mathrm{C}(21)-\mathrm{C}(22)-\mathrm{C}(23)-\mathrm{O}(10)$ & $-179.9(4)$ \\
\hline $\mathrm{C}(21)-\mathrm{C}(22)-\mathrm{C}(23)-\mathrm{C}(24)$ & $0.3(5)$ \\
\hline $\mathrm{O}(10)-\mathrm{C}(23)-\mathrm{C}(24)-\mathrm{N}(3)$ & $-178.9(4)$ \\
\hline $\mathrm{C}(22)-\mathrm{C}(23)-\mathrm{C}(24)-\mathrm{N}(3)$ & $0.9(6)$ \\
\hline $\mathrm{C}(22)-\mathrm{C}(23)-\mathrm{C}(24)-\mathrm{C}(28)$ & $175.8(5)$ \\
\hline $\mathrm{O}(10)-\mathrm{C}(23)-\mathrm{C}(24)-\mathrm{C}(28)$ & $-4.0(9)$ \\
\hline $\mathrm{C}(23)-\mathrm{C}(24)-\mathrm{C}(28)-\mathrm{O}(12)$ & $1.6(8)$ \\
\hline $\mathrm{C}(23)-\mathrm{C}(24)-\mathrm{C}(28)-\mathrm{O}(11)$ & $-178.8(5)$ \\
\hline $\mathrm{N}(3)-\mathrm{C}(24)-\mathrm{C}(28)-\mathrm{O}(11)$ & $-4.3(7)$ \\
\hline $\mathrm{N}(3)-\mathrm{C}(24)-\mathrm{C}(28)-\mathrm{O}(12)$ & $176.0(4)$ \\
\hline
\end{tabular}


Table 7. Hydrogen-bonds for compound 3 [A and deg.].

\begin{tabular}{c|c|c|c|c} 
D-H...A & $\mathrm{d}(\mathrm{D}-\mathrm{H})$ & $\mathrm{d}(\mathrm{H} . . . \mathrm{A})$ & $\mathrm{d}(\mathrm{D} \ldots \mathrm{A})$ & $<(\mathrm{DHA})$ \\
\hline $\mathrm{N}(1)-\mathrm{H}(1) \ldots \mathrm{O}(11)$ & 0.8808 & 1.9530 & $2.807(4)$ & 163.05 \\
\hline $\mathrm{N}(2)-\mathrm{H}(2) \ldots \mathrm{O}(7) \# 1$ & 0.8796 & 2.0297 & $2.895(5)$ & 167.57 \\
\hline $\mathrm{N}(3)-\mathrm{H}(3) \ldots \mathrm{O}(3)$ & 0.8798 & 2.0369 & $2.852(4)$ & 153.58 \\
\hline $\mathrm{C}(29)-\mathrm{H}(29 \mathrm{~A}) \ldots \mathrm{O}(11)$ & 0.9903 & 2.2553 & $2.658(8)$ & 102.92 \\
\hline $\mathrm{C}(30)-\mathrm{H}(30 \mathrm{~B}) \ldots \mathrm{O}(6) \# 2$ & 0.9793 & 2.5664 & $3.497(11)$ & 158.79
\end{tabular}




\section{Boc-MOPAS-OEt, 5.}

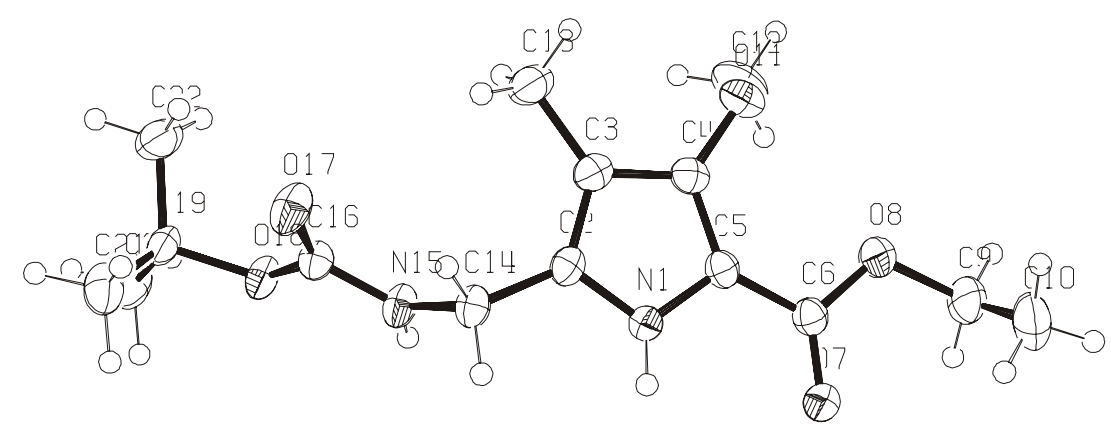

Table 1. Crystal data and structure refinement for compound $\mathbf{5}$.

\begin{tabular}{|c|c|}
\hline Crystal Data & \\
\hline Empirical formula & C15 H24 N2 O5 \\
\hline Formula weight & 312.36 \\
\hline Crystal size & $0.50 \times 0.48 \times 0.32 \mathrm{~mm}$ \\
\hline Crystal description & prism \\
\hline Crystal colour & translucent colourless \\
\hline Crystal system & Monoclinic \\
\hline Space group & $\mathrm{P} 21 / \mathrm{n}$ \\
\hline \multirow[t]{3}{*}{ Unit cell dimensions } & $\mathrm{a}=11.9505(8) \mathrm{A}$ alpha $=90 \mathrm{deg}$. \\
\hline & $\mathrm{b}=9.4792(8) \mathrm{A} \quad$ beta $=102.832(8) \mathrm{deg}$ \\
\hline & $\mathrm{c}=15.5098(10) \mathrm{A}$ gamma $=90 \mathrm{deg}$. \\
\hline Volume & $1713.1(2) \mathrm{A}^{\wedge} 3$ \\
\hline Z, Calculated density & $4,1.211 \mathrm{Mg} / \mathrm{m}^{\wedge} 3$ \\
\hline Absorption coefficient & $0.091 \mathrm{~mm}^{\wedge}-1$ \\
\hline $\mathrm{F}(000)$ & 672 \\
\hline \multicolumn{2}{|l|}{ Data Collection } \\
\hline Measurement device type & STOE-IPDS diffractometer \\
\hline Measuremnet method & rotation \\
\hline Temperature & $173(1) \mathrm{K}$ \\
\hline Wavelength & $0.71073 \mathrm{~A}$ \\
\hline Monochromator & graphite \\
\hline Theta range for data collection & 1.96 to $25.79 \mathrm{deg}$. \\
\hline Index ranges & $-14<=\mathrm{h}<=14,-11<=\mathrm{k}<=11,-18<=\mathrm{l}<=18$ \\
\hline Reflections collected / unique & $23264 / 3273[\mathrm{R}($ int $)=0.0679]$ \\
\hline Reflections greater $\mathrm{I}>4 \| \mathrm{s}(\mathrm{I})$ & 2740 \\
\hline Absorption correction & None \\
\hline Refinement & \\
\hline
\end{tabular}




\begin{tabular}{c|c} 
Refinement method & Full-matrix least-squares on $\mathrm{F}^{\wedge} 2$ \\
\hline Hydrogen treatment & mixed \\
\hline Data / restraints / parameters & $3273 / 0 / 259$ \\
\hline Goodness-of-fit on $\mathrm{F}^{\wedge} 2$ & $\mathrm{R} 1=0.0367, \mathrm{wR} 2=0.0970$ \\
\hline Final R indices [I>2sigma(I)] & $\mathrm{R} 1=0.0449, \mathrm{wR} 2=0.1010$ \\
\hline R indices (all data) & $?$ \\
\hline Absolute structure parameter & 0.283 and $-0.238 \mathrm{e} . \mathrm{A}^{\wedge}-3$
\end{tabular}

Table 2. Atomic coordinates ( x 10^4) and equivalent isotropic displacement parameters $\left(\mathrm{A}^{\wedge} 2 \times 10^{\wedge} 3\right)$ for compound 5 . $\mathrm{U}(\mathrm{eq})$ is defined as one third of the trace of the orthogonalized Uij tensor.

\begin{tabular}{|c|c|c|c|c|}
\hline & $\mathrm{X}$ & $\mathrm{y}$ & $\mathrm{Z}$ & $\mathrm{U}(\mathrm{eq})$ \\
\hline $\mathrm{O}(7)$ & $9220(1)$ & $-530(1)$ & $-1087(1)$ & $34(1)$ \\
\hline $\mathrm{O}(8)$ & $7586(1)$ & $-31(1)$ & $-2078(1)$ & $42(1)$ \\
\hline $\mathrm{O}(11)$ & $5839(1)$ & 1588(1) & $-1389(1)$ & $37(1)$ \\
\hline $\mathrm{O}(17)$ & $7144(1)$ & $3184(1)$ & $2892(1)$ & $41(1)$ \\
\hline $\mathrm{O}(18)$ & $6968(1)$ & $966(1)$ & $3411(1)$ & $31(1)$ \\
\hline $\mathrm{N}(1)$ & $8418(1)$ & $956(1)$ & $213(1)$ & $26(1)$ \\
\hline $\mathrm{N}(15)$ & $7790(1)$ & $1254(1)$ & $2281(1)$ & $30(1)$ \\
\hline $\mathrm{C}(2)$ & $7802(1)$ & $1669(1)$ & $709(1)$ & $27(1)$ \\
\hline $\mathrm{C}(3)$ & $6736(1)$ & $2000(1)$ & $182(1)$ & $30(1)$ \\
\hline$C(4)$ & $6730(1)$ & $1448(1)$ & $-663(1)$ & $28(1)$ \\
\hline$C(5)$ & 7784(1) & $801(1)$ & $-639(1)$ & $27(1)$ \\
\hline$C(6)$ & $8276(1)$ & $27(1)$ & $-1270(1)$ & $28(1)$ \\
\hline $\mathrm{C}(9)$ & $8042(2)$ & $-789(2)$ & $-2752(1)$ & $48(1)$ \\
\hline$C(10)$ & $8841(2)$ & 112(2) & $-3125(1)$ & $58(1)$ \\
\hline$C(12)$ & $5055(2)$ & $434(2)$ & $-1510(1)$ & $54(1)$ \\
\hline$C(13)$ & $5805(1)$ & $2849(2)$ & $445(1)$ & $41(1)$ \\
\hline $\mathrm{C}(14)$ & $8304(1)$ & $2033(1)$ & $1657(1)$ & $29(1)$ \\
\hline$C(16)$ & 7291(1) & 1914(1) & $2862(1)$ & $28(1)$ \\
\hline$C(19)$ & $6526(1)$ & $1454(1)$ & $4177(1)$ & $31(1)$ \\
\hline $\mathrm{C}(20)$ & $6372(2)$ & $79(2)$ & $4642(1)$ & $41(1)$ \\
\hline $\mathrm{C}(21)$ & $7423(2)$ & $2358(2)$ & $4777(1)$ & $44(1)$ \\
\hline$C(22)$ & $5390(2)$ & $2207(2)$ & $3870(1)$ & $48(1)$ \\
\hline
\end{tabular}


Table 3. Bond lengths $[\mathrm{A}]$ and angles [deg] for compound $\mathbf{5}$.

\begin{tabular}{c|c}
$\mathrm{O}(7)-\mathrm{C}(6)$ & $1.2203(16)$ \\
\hline $\mathrm{O}(8)-\mathrm{C}(6)$ & $1.3395(16)$ \\
\hline $\mathrm{O}(8)-\mathrm{C}(9)$ & $1.469(2)$ \\
\hline $\mathrm{O}(11)-\mathrm{C}(4)$ & $1.3733(16)$ \\
\hline $\mathrm{O}(11)-\mathrm{C}(12)$ & $1.425(2)$ \\
\hline $\mathrm{O}(17)-\mathrm{C}(16)$ & $1.2185(14)$ \\
\hline $\mathrm{O}(18)-\mathrm{C}(16)$ & $1.3524(15)$ \\
\hline $\mathrm{O}(18)-\mathrm{C}(19)$ & $1.4777(16)$ \\
\hline $\mathrm{N}(1)-\mathrm{C}(2)$ & $1.3577(17)$ \\
\hline $\mathrm{N}(1)-\mathrm{C}(5)$ & $1.3773(16)$ \\
\hline $\mathrm{N}(15)-\mathrm{C}(14)$ & $1.4574(17)$ \\
\hline $\mathrm{N}(15)-\mathrm{C}(16)$ & $1.3411(17)$ \\
\hline $\mathrm{N}(1)-\mathrm{H}(1)$ & $0.867(18)$ \\
\hline $\mathrm{N}(15)-\mathrm{H}(15)$ & $0.827(18)$ \\
\hline $\mathrm{C}(2)-\mathrm{C}(3)$ & $1.3885(18)$ \\
\hline $\mathrm{C}(2)-\mathrm{C}(14)$ & $1.4992(17)$ \\
\hline $\mathrm{C}(3)-\mathrm{C}(4)$ & $1.4098(17)$ \\
\hline $\mathrm{C}(3)-\mathrm{C}(13)$ & $1.501(2)$ \\
\hline $\mathrm{C}(4)-\mathrm{C}(5)$ & $1.3943(19)$ \\
\hline $\mathrm{C}(5)-\mathrm{C}(6)$ & $1.4485(18)$ \\
\hline $\mathrm{C}(9)-\mathrm{C}(10)$ & $1.492(3)$ \\
\hline $\mathrm{C}(19)-\mathrm{C}(22)$ & $1.514(2)$ \\
\hline $\mathrm{C}(19)-\mathrm{C}(20)$ & $1.521(2)$ \\
\hline $\mathrm{C}(19)-\mathrm{C}(21)$ & $1.519(2)$ \\
\hline $\mathrm{C}(9)-\mathrm{H}(9 \mathrm{~A})$ & $0.960(19)$ \\
\hline $\mathrm{C}(9)-\mathrm{H}(9 \mathrm{~B})$ & $0.957(19)$ \\
\hline $\mathrm{C}(10)-\mathrm{H}(10 \mathrm{~A})$ & 0.9603 \\
\hline $\mathrm{C}(10)-\mathrm{H}(10 \mathrm{~B})$ & 0.9600 \\
\hline $\mathrm{C}(10)-\mathrm{H}(10 \mathrm{C})$ & 0.9598 \\
\hline $\mathrm{C}(12)-\mathrm{H}(12 \mathrm{~A})$ & 0.9599 \\
\hline $\mathrm{C}(12)-\mathrm{H}(12 \mathrm{~B})$ & 0.9599 \\
\hline $\mathrm{C}(12)-\mathrm{H}(12 \mathrm{C})$ & 0.9600 \\
\hline $\mathrm{C}(13)-\mathrm{H}(13 \mathrm{~A})$ & 0.9598 \\
\hline $\mathrm{C}(13)-\mathrm{H}(13 \mathrm{~B})$ & 0.9595 \\
\hline $\mathrm{C}(13)-\mathrm{H}(13 \mathrm{C})$ & 0.9608 \\
\hline $\mathrm{C}(14)-\mathrm{H}(14 \mathrm{~A})$ & $0.979(15)$ \\
\hline & $0.981(17)$ \\
\hline
\end{tabular}




\begin{tabular}{c|c}
$\mathrm{C}(20)-\mathrm{H}(20 \mathrm{~B})$ & $0.942(19)$ \\
\hline $\mathrm{C}(20)-\mathrm{H}(20 \mathrm{C})$ & $0.990(18)$ \\
\hline $\mathrm{C}(21)-\mathrm{H}(21 \mathrm{~A})$ & $0.956(19)$ \\
\hline $\mathrm{C}(21)-\mathrm{H}(21 \mathrm{~B})$ & $0.99(2)$ \\
\hline $\mathrm{C}(21)-\mathrm{H}(21 \mathrm{C})$ & $1.03(2)$ \\
\hline $\mathrm{C}(22)-\mathrm{H}(22 \mathrm{~A})$ & $0.971(19)$ \\
\hline $\mathrm{C}(22)-\mathrm{H}(22 \mathrm{~B})$ & $0.98(2)$ \\
\hline $\mathrm{C}(22)-\mathrm{H}(22 \mathrm{C})$ & $0.98(2)$
\end{tabular}

\begin{tabular}{c|c}
$\mathrm{C}(6)-\mathrm{O}(8)-\mathrm{C}(9)$ & $115.89(12)$ \\
\hline $\mathrm{C}(4)-\mathrm{O}(11)-\mathrm{C}(12)$ & $113.59(11)$ \\
\hline $\mathrm{C}(16)-\mathrm{O}(18)-\mathrm{C}(19)$ & $120.06(9)$ \\
\hline $\mathrm{C}(2)-\mathrm{N}(1)-\mathrm{C}(5)$ & $110.17(11)$ \\
\hline $\mathrm{C}(14)-\mathrm{N}(15)-\mathrm{C}(16)$ & $121.66(11)$ \\
\hline $\mathrm{C}(2)-\mathrm{N}(1)-\mathrm{H}(1)$ & $123.9(10)$ \\
\hline $\mathrm{C}(5)-\mathrm{N}(1)-\mathrm{H}(1)$ & $125.8(10)$ \\
\hline $\mathrm{C}(16)-\mathrm{N}(15)-\mathrm{H}(15)$ & $117.7(11)$ \\
\hline $\mathrm{C}(14)-\mathrm{N}(15)-\mathrm{H}(15)$ & $120.4(11)$ \\
\hline $\mathrm{N}(1)-\mathrm{C}(2)-\mathrm{C}(14)$ & $121.40(12)$ \\
\hline $\mathrm{C}(3)-\mathrm{C}(2)-\mathrm{C}(14)$ & $129.84(12)$ \\
\hline $\mathrm{N}(1)-\mathrm{C}(2)-\mathrm{C}(3)$ & $108.71(11)$ \\
\hline $\mathrm{C}(2)-\mathrm{C}(3)-\mathrm{C}(4)$ & $106.17(11)$ \\
\hline $\mathrm{C}(2)-\mathrm{C}(3)-\mathrm{C}(13)$ & $126.81(12)$ \\
\hline $\mathrm{C}(4)-\mathrm{C}(3)-\mathrm{C}(13)$ & $126.93(12)$ \\
\hline $\mathrm{O}(11)-\mathrm{C}(4)-\mathrm{C}(3)$ & $124.68(12)$ \\
\hline $\mathrm{C}(3)-\mathrm{C}(4)-\mathrm{C}(5)$ & $108.66(11)$ \\
\hline $\mathrm{O}(11)-\mathrm{C}(4)-\mathrm{C}(5)$ & $126.60(11)$ \\
\hline $\mathrm{N}(1)-\mathrm{C}(5)-\mathrm{C}(4)$ & $106.29(11)$ \\
\hline $\mathrm{N}(1)-\mathrm{C}(5)-\mathrm{C}(6)$ & $118.88(12)$ \\
\hline $\mathrm{C}(4)-\mathrm{C}(5)-\mathrm{C}(6)$ & $134.79(12)$ \\
\hline $\mathrm{O}(8)-\mathrm{C}(6)-\mathrm{C}(5)$ & $113.23(11)$ \\
\hline $\mathrm{O}(7)-\mathrm{C}(6)-\mathrm{O}(8)$ & $123.07(12)$ \\
\hline $\mathrm{O}(7)-\mathrm{C}(6)-\mathrm{C}(5)$ & $123.70(11)$ \\
\hline $\mathrm{O}(8)-\mathrm{C}(9)-\mathrm{C}(10)$ & $111.56(15)$ \\
\hline $\mathrm{N}(15)-\mathrm{C}(14)-\mathrm{C}(2)$ & $113.48(11)$ \\
\hline $\mathrm{O}(17)-\mathrm{C}(16)-\mathrm{O}(18)$ & $124.67(12)$ \\
\hline $\mathrm{O}(17)-\mathrm{C}(16)-\mathrm{N}(15)$ & $125.21(12)$ \\
\hline $\mathrm{O}(18)-\mathrm{C}(16)-\mathrm{N}(15)$ & $110.13(10)$ \\
\hline $\mathrm{C}(20)-\mathrm{C}(19)-\mathrm{C}(22)$ & $111.02(13)$ \\
\hline $\mathrm{C}(21)-\mathrm{C}(19)-\mathrm{C}(22)$ & $112.86(12)$ \\
\end{tabular}




\begin{tabular}{|c|c|}
\hline$C(20)-C(19)-C(21)$ & $109.62(11)$ \\
\hline $\mathrm{O}(18)-\mathrm{C}(19)-\mathrm{C}(20)$ & $102.43(10)$ \\
\hline $\mathrm{O}(18)-\mathrm{C}(19)-\mathrm{C}(21)$ & $109.89(12)$ \\
\hline $\mathrm{O}(18)-\mathrm{C}(19)-\mathrm{C}(22)$ & $110.53(11)$ \\
\hline $\mathrm{O}(8)-\mathrm{C}(9)-\mathrm{H}(9 \mathrm{~A})$ & $102.1(11)$ \\
\hline $\mathrm{O}(8)-\mathrm{C}(9)-\mathrm{H}(9 \mathrm{~B})$ & $108.6(11)$ \\
\hline $\mathrm{C}(10)-\mathrm{C}(9)-\mathrm{H}(9 \mathrm{~A})$ & $112.4(11)$ \\
\hline $\mathrm{C}(10)-\mathrm{C}(9)-\mathrm{H}(9 \mathrm{~B})$ & $109.4(12)$ \\
\hline $\mathrm{H}(9 \mathrm{~A})-\mathrm{C}(9)-\mathrm{H}(9 \mathrm{~B})$ & $112.7(15)$ \\
\hline $\mathrm{C}(9)-\mathrm{C}(10)-\mathrm{H}(10 \mathrm{~A})$ & 109.49 \\
\hline $\mathrm{C}(9)-\mathrm{C}(10)-\mathrm{H}(10 \mathrm{~B})$ & 109.45 \\
\hline $\mathrm{C}(9)-\mathrm{C}(10)-\mathrm{H}(10 \mathrm{C})$ & 109.52 \\
\hline $\mathrm{H}(10 \mathrm{~A})-\mathrm{C}(10)-\mathrm{H}(10 \mathrm{~B})$ & 109.45 \\
\hline $\mathrm{H}(10 \mathrm{~A})-\mathrm{C}(10)-\mathrm{H}(10 \mathrm{C})$ & 109.42 \\
\hline $\mathrm{H}(10 \mathrm{~B})-\mathrm{C}(10)-\mathrm{H}(10 \mathrm{C})$ & 109.49 \\
\hline $\mathrm{O}(11)-\mathrm{C}(12)-\mathrm{H}(12 \mathrm{~A})$ & 109.45 \\
\hline $\mathrm{O}(11)-\mathrm{C}(12)-\mathrm{H}(12 \mathrm{~B})$ & 109.46 \\
\hline $\mathrm{O}(11)-\mathrm{C}(12)-\mathrm{H}(12 \mathrm{C})$ & 109.44 \\
\hline $\mathrm{H}(12 \mathrm{~A})-\mathrm{C}(12)-\mathrm{H}(12 \mathrm{~B})$ & 109.51 \\
\hline $\mathrm{H}(12 \mathrm{~A})-\mathrm{C}(12)-\mathrm{H}(12 \mathrm{C})$ & 109.45 \\
\hline $\mathrm{H}(12 \mathrm{~B})-\mathrm{C}(12)-\mathrm{H}(12 \mathrm{C})$ & 109.52 \\
\hline $\mathrm{C}(3)-\mathrm{C}(13)-\mathrm{H}(13 \mathrm{~A})$ & 109.49 \\
\hline $\mathrm{C}(3)-\mathrm{C}(13)-\mathrm{H}(13 \mathrm{~B})$ & 109.51 \\
\hline $\mathrm{C}(3)-\mathrm{C}(13)-\mathrm{H}(13 \mathrm{C})$ & 109.46 \\
\hline $\mathrm{H}(13 \mathrm{~A})-\mathrm{C}(13)-\mathrm{H}(13 \mathrm{~B})$ & 109.41 \\
\hline $\mathrm{H}(13 \mathrm{~A})-\mathrm{C}(13)-\mathrm{H}(13 \mathrm{C})$ & 109.49 \\
\hline $\mathrm{H}(13 \mathrm{~B})-\mathrm{C}(13)-\mathrm{H}(13 \mathrm{C})$ & 109.47 \\
\hline $\mathrm{N}(15)-\mathrm{C}(14)-\mathrm{H}(14 \mathrm{~A})$ & $106.8(9)$ \\
\hline $\mathrm{N}(15)-\mathrm{C}(14)-\mathrm{H}(14 \mathrm{~B})$ & $108.6(9)$ \\
\hline $\mathrm{C}(2)-\mathrm{C}(14)-\mathrm{H}(14 \mathrm{~A})$ & $109.8(8)$ \\
\hline $\mathrm{C}(2)-\mathrm{C}(14)-\mathrm{H}(14 \mathrm{~B})$ & $109.9(9)$ \\
\hline $\mathrm{H}(14 \mathrm{~A})-\mathrm{C}(14)-\mathrm{H}(14 \mathrm{~B})$ & $108.1(13)$ \\
\hline $\mathrm{C}(19)-\mathrm{C}(20)-\mathrm{H}(20 \mathrm{~A})$ & $111.5(10)$ \\
\hline $\mathrm{C}(19)-\mathrm{C}(20)-\mathrm{H}(20 \mathrm{~B})$ & $108.3(10)$ \\
\hline $\mathrm{C}(19)-\mathrm{C}(20)-\mathrm{H}(20 \mathrm{C})$ & $108.0(11)$ \\
\hline $\mathrm{H}(20 \mathrm{~A})-\mathrm{C}(20)-\mathrm{H}(20 \mathrm{~B})$ & $109.6(15)$ \\
\hline $\mathrm{H}(20 \mathrm{~A})-\mathrm{C}(20)-\mathrm{H}(20 \mathrm{C})$ & $110.7(15)$ \\
\hline $\mathrm{H}(20 \mathrm{~B})-\mathrm{C}(20)-\mathrm{H}(20 \mathrm{C})$ & $108.7(16)$ \\
\hline $\mathrm{C}(19)-\mathrm{C}(21)-\mathrm{H}(21 \mathrm{~A})$ & $113.6(11)$ \\
\hline $\mathrm{C}(19)-\mathrm{C}(21)-\mathrm{H}(21 \mathrm{~B})$ & $107.7(12)$ \\
\hline
\end{tabular}




\begin{tabular}{c|l}
$\mathrm{C}(19)-\mathrm{C}(21)-\mathrm{H}(21 \mathrm{C})$ & $110.0(11)$ \\
\hline $\mathrm{H}(21 \mathrm{~A})-\mathrm{C}(21)-\mathrm{H}(21 \mathrm{~B})$ & $109.5(16)$ \\
\hline $\mathrm{H}(21 \mathrm{~A})-\mathrm{C}(21)-\mathrm{H}(21 \mathrm{C})$ & $108.3(16)$ \\
\hline $\mathrm{H}(21 \mathrm{~B})-\mathrm{C}(21)-\mathrm{H}(21 \mathrm{C})$ & $107.5(16)$ \\
\hline $\mathrm{C}(19)-\mathrm{C}(22)-\mathrm{H}(22 \mathrm{~A})$ & $112.8(12)$ \\
\hline $\mathrm{C}(19)-\mathrm{C}(22)-\mathrm{H}(22 \mathrm{~B})$ & $110.1(12)$ \\
\hline $\mathrm{C}(19)-\mathrm{C}(22)-\mathrm{H}(22 \mathrm{C})$ & $108.9(12)$ \\
\hline $\mathrm{H}(22 \mathrm{~A})-\mathrm{C}(22)-\mathrm{H}(22 \mathrm{~B})$ & $109.7(16)$ \\
\hline $\mathrm{H}(22 \mathrm{~A})-\mathrm{C}(22)-\mathrm{H}(22 \mathrm{C})$ & $107.3(16)$ \\
\hline $\mathrm{H}(22 \mathrm{~B})-\mathrm{C}(22)-\mathrm{H}(22 \mathrm{C})$ & $107.8(17)$
\end{tabular}

Table 4. Anisotropic displacement parameters $\left(\mathrm{A}^{\wedge} 2 \mathrm{x} 10^{\wedge} 3\right)$ for compound 5 . The anisotropic displacement factor exponent takes the form: $-2 \mathrm{pi}^{\wedge} 2\left[\mathrm{~h}^{\wedge} 2 \mathrm{a}^{* \wedge} 2 \mathrm{U} 11+\ldots+2 \mathrm{~h} \mathrm{k} \mathrm{a} \mathrm{b}^{*} \mathrm{U} 12\right.$ ]

\begin{tabular}{c|c|c|c|c|c|c} 
& $\mathrm{U} 11$ & $\mathrm{U} 22$ & $\mathrm{U} 33$ & $\mathrm{U} 23$ & $\mathrm{U} 13$ & $\mathrm{U} 12$ \\
\hline & & & & & & \\
\hline $\mathrm{O}(7)$ & $31(1)$ & $40(1)$ & $30(1)$ & $-3(1)$ & $8(1)$ & $4(1)$ \\
\hline $\mathrm{O}(8)$ & $37(1)$ & $61(1)$ & $27(1)$ & $-10(1)$ & $2(1)$ & $8(1)$ \\
\hline $\mathrm{O}(11)$ & $31(1)$ & $38(1)$ & $37(1)$ & $9(1)$ & $1(1)$ & $0(1)$ \\
\hline $\mathrm{O}(17)$ & $63(1)$ & $22(1)$ & $46(1)$ & $1(1)$ & $29(1)$ & $3(1)$ \\
\hline $\mathrm{O}(18)$ & $43(1)$ & $23(1)$ & $32(1)$ & $-1(1)$ & $20(1)$ & $0(1)$ \\
\hline $\mathrm{N}(1)$ & $25(1)$ & $28(1)$ & $25(1)$ & $1(1)$ & $7(1)$ & $1(1)$ \\
\hline $\mathrm{N}(15)$ & $44(1)$ & $20(1)$ & $30(1)$ & $0(1)$ & $17(1)$ & $0(1)$ \\
\hline $\mathrm{C}(2)$ & $31(1)$ & $23(1)$ & $29(1)$ & $1(1)$ & $13(1)$ & $-2(1)$ \\
\hline $\mathrm{C}(3)$ & $30(1)$ & $25(1)$ & $36(1)$ & $5(1)$ & $12(1)$ & $0(1)$ \\
\hline $\mathrm{C}(4)$ & $28(1)$ & $26(1)$ & $31(1)$ & $5(1)$ & $7(1)$ & $-2(1)$ \\
\hline $\mathrm{C}(5)$ & $30(1)$ & $28(1)$ & $24(1)$ & $3(1)$ & $7(1)$ & $-2(1)$ \\
\hline $\mathrm{C}(6)$ & $29(1)$ & $30(1)$ & $27(1)$ & $1(1)$ & $7(1)$ & $-2(1)$ \\
\hline $\mathrm{C}(9)$ & $46(1)$ & $65(1)$ & $30(1)$ & $-17(1)$ & $5(1)$ & $2(1)$ \\
\hline $\mathrm{C}(10)$ & $74(1)$ & $72(1)$ & $32(1)$ & $-2(1)$ & $17(1)$ & $4(1)$ \\
\hline $\mathrm{C}(12)$ & $41(1)$ & $55(1)$ & $55(1)$ & $9(1)$ & $-9(1)$ & $-12(1)$ \\
\hline $\mathrm{C}(13)$ & $37(1)$ & $41(1)$ & $48(1)$ & $4(1)$ & $18(1)$ & $9(1)$ \\
\hline $\mathrm{C}(14)$ & $35(1)$ & $27(1)$ & $28(1)$ & $-1(1)$ & $12(1)$ & $-3(1)$ \\
\hline $\mathrm{C}(16)$ & $33(1)$ & $23(1)$ & $28(1)$ & $-1(1)$ & $10(1)$ & $-1(1)$ \\
\hline $\mathrm{C}(19)$ & $38(1)$ & $30(1)$ & $30(1)$ & $-1(1)$ & $17(1)$ & $6(1)$ \\
\hline $\mathrm{C}(20)$ & $54(1)$ & $37(1)$ & $40(1)$ & $4(1)$ & $28(1)$ & $4(1)$ \\
\hline $\mathrm{C}(21)$ & $58(1)$ & $41(1)$ & $35(1)$ & $-6(1)$ & $13(1)$ & $-1(1)$ \\
\hline $\mathrm{C}(22)$ & $44(1)$ & $53(1)$ & $52(1)$ & $8(1)$ & $21(1)$ & $16(1)$ \\
& & & & & &
\end{tabular}


Table 5. Hydrogen coordinates ( $\left.\mathrm{x} 10^{\wedge} 4\right)$ and isotropic displacement parameters $\left(\mathrm{A}^{\wedge} 2 \mathrm{x}\right.$ $10^{\wedge} 3$ ) for compound 5.

\begin{tabular}{|c|c|c|c|c|}
\hline & $\mathrm{X}$ & $\mathrm{y}$ & Z & $\mathrm{U}(\mathrm{eq})$ \\
\hline $\mathrm{H}(1)$ & $9123(15)$ & $689(15)$ & $406(10)$ & $32(4)$ \\
\hline $\mathrm{H}(9 \mathrm{~A})$ & $7355(16)$ & $-1016(18)$ & $-3182(12)$ & $47(5)$ \\
\hline $\mathrm{H}(9 \mathrm{~B})$ & $8441(16)$ & $-1610(20)$ & $-2485(12)$ & $50(5)$ \\
\hline $\mathrm{H}(10 \mathrm{~A})$ & 9041 & -363 & -3617 & 70 \\
\hline $\mathrm{H}(10 \mathrm{~B})$ & 8474 & 992 & -3321 & 70 \\
\hline $\mathrm{H}(10 \mathrm{C})$ & 9524 & 287 & -2679 & 70 \\
\hline $\mathrm{H}(12 \mathrm{~A})$ & 5455 & -420 & -1585 & 64 \\
\hline $\mathrm{H}(12 \mathrm{~B})$ & 4728 & 348 & -1000 & 64 \\
\hline $\mathrm{H}(12 \mathrm{C})$ & 4456 & 596 & -2025 & 64 \\
\hline $\mathrm{H}(13 \mathrm{~A})$ & 5280 & 2227 & 642 & 49 \\
\hline $\mathrm{H}(13 \mathrm{~B})$ & 5401 & 3382 & -54 & 49 \\
\hline $\mathrm{H}(13 \mathrm{C})$ & 6140 & 3483 & 915 & 49 \\
\hline $\mathrm{H}(14 \mathrm{~A})$ & $8183(13)$ & $3036(16)$ & 1757(9) & $31(4)$ \\
\hline $\mathrm{H}(14 \mathrm{~B})$ & $9133(14)$ & $1854(15)$ & 1796(10) & $33(4)$ \\
\hline $\mathrm{H}(15)$ & $7851(13)$ & $385(19)$ & $2317(10)$ & $35(4)$ \\
\hline $\mathrm{H}(20 \mathrm{~A})$ & $7117(16)$ & $-457(18)$ & $4816(11)$ & $47(5)$ \\
\hline $\mathrm{H}(20 \mathrm{~B})$ & $6097(15)$ & 293(18) & $5151(13)$ & $47(4)$ \\
\hline $\mathrm{H}(20 \mathrm{C})$ & $5786(16)$ & $-491(19)$ & $4237(12)$ & $49(5)$ \\
\hline $\mathrm{H}(21 \mathrm{~A})$ & $7573(16)$ & $3230(20)$ & $4515(12)$ & $56(5)$ \\
\hline $\mathrm{H}(21 \mathrm{~B})$ & $7147(17)$ & $2550(20)$ & $5324(14)$ & $61(5)$ \\
\hline $\mathrm{H}(21 \mathrm{C})$ & 8181(18) & $1810(20)$ & $4957(13)$ & $63(6)$ \\
\hline $\mathrm{H}(22 \mathrm{~A})$ & $5462(16)$ & $3090(20)$ & $3570(12)$ & $51(5)$ \\
\hline $\mathrm{H}(22 \mathrm{~B})$ & $5035(17)$ & $2380(20)$ & $4373(14)$ & $61(5)$ \\
\hline $\mathrm{H}(22 \mathrm{C})$ & $4870(17)$ & $1600(20)$ & $3449(13)$ & $59(5)$ \\
\hline
\end{tabular}

Table 6. Torsion angles [deg] for compound $\mathbf{5}$.

\begin{tabular}{c|c}
$\mathrm{C}(9)-\mathrm{O}(8)-\mathrm{C}(6)-\mathrm{C}(5)$ & $179.32(12)$ \\
\hline $\mathrm{C}(6)-\mathrm{O}(8)-\mathrm{C}(9)-\mathrm{C}(10)$ & $-80.67(16)$ \\
\hline $\mathrm{C}(9)-\mathrm{O}(8)-\mathrm{C}(6)-\mathrm{O}(7)$ & $-1.30(19)$ \\
\hline $\mathrm{C}(12)-\mathrm{O}(11)-\mathrm{C}(4)-\mathrm{C}(5)$ & $89.25(16)$ \\
\hline $\mathrm{C}(12)-\mathrm{O}(11)-\mathrm{C}(4)-\mathrm{C}(3)$ & $-93.55(15)$ \\
\hline $\mathrm{C}(16)-\mathrm{O}(18)-\mathrm{C}(19)-\mathrm{C}(20)$ & $-175.33(12)$ \\
\hline $\mathrm{C}(19)-\mathrm{O}(18)-\mathrm{C}(16)-\mathrm{N}(15)$ & $172.53(11)$
\end{tabular}




\begin{tabular}{c|c}
$\mathrm{C}(19)-\mathrm{O}(18)-\mathrm{C}(16)-\mathrm{O}(17)$ & $-7.65(19)$ \\
\hline $\mathrm{C}(16)-\mathrm{O}(18)-\mathrm{C}(19)-\mathrm{C}(21)$ & $-58.89(15)$ \\
\hline $\mathrm{C}(16)-\mathrm{O}(18)-\mathrm{C}(19)-\mathrm{C}(22)$ & $66.32(15)$ \\
\hline $\mathrm{C}(2)-\mathrm{N}(1)-\mathrm{C}(5)-\mathrm{C}(6)$ & $-178.11(11)$ \\
\hline $\mathrm{C}(2)-\mathrm{N}(1)-\mathrm{C}(5)-\mathrm{C}(4)$ & $-0.08(14)$ \\
\hline $\mathrm{C}(5)-\mathrm{N}(1)-\mathrm{C}(2)-\mathrm{C}(14)$ & $-177.69(11)$ \\
\hline $\mathrm{C}(5)-\mathrm{N}(1)-\mathrm{C}(2)-\mathrm{C}(3)$ & $-0.08(14)$ \\
\hline $\mathrm{C}(14)-\mathrm{N}(15)-\mathrm{C}(16)-\mathrm{O}(17)$ & $5.2(2)$ \\
\hline $\mathrm{C}(16)-\mathrm{N}(15)-\mathrm{C}(14)-\mathrm{C}(2)$ & $-122.19(13)$ \\
\hline $\mathrm{C}(14)-\mathrm{N}(15)-\mathrm{C}(16)-\mathrm{O}(18)$ & $-174.98(11)$ \\
\hline $\mathrm{N}(1)-\mathrm{C}(2)-\mathrm{C}(14)-\mathrm{N}(15)$ & $-110.78(14)$ \\
\hline $\mathrm{C}(14)-\mathrm{C}(2)-\mathrm{C}(3)-\mathrm{C}(4)$ & $177.55(12)$ \\
\hline $\mathrm{N}(1)-\mathrm{C}(2)-\mathrm{C}(3)-\mathrm{C}(4)$ & $0.21(14)$ \\
\hline $\mathrm{N}(1)-\mathrm{C}(2)-\mathrm{C}(3)-\mathrm{C}(13)$ & $-176.52(12)$ \\
\hline $\mathrm{C}(14)-\mathrm{C}(2)-\mathrm{C}(3)-\mathrm{C}(13)$ & $0.8(2)$ \\
\hline $\mathrm{C}(3)-\mathrm{C}(2)-\mathrm{C}(14)-\mathrm{N}(15)$ & $72.17(17)$ \\
\hline $\mathrm{C}(2)-\mathrm{C}(3)-\mathrm{C}(4)-\mathrm{C}(5)$ & $-0.26(14)$ \\
\hline $\mathrm{C}(2)-\mathrm{C}(3)-\mathrm{C}(4)-\mathrm{O}(11)$ & $-177.89(11)$ \\
\hline $\mathrm{C}(13)-\mathrm{C}(3)-\mathrm{C}(4)-\mathrm{O}(11)$ & $-1.2(2)$ \\
\hline $\mathrm{C}(13)-\mathrm{C}(3)-\mathrm{C}(4)-\mathrm{C}(5)$ & $176.46(13)$ \\
\hline $\mathrm{O}(11)-\mathrm{C}(4)-\mathrm{C}(5)-\mathrm{C}(6)$ & $-4.7(2)$ \\
\hline $\mathrm{O}(11)-\mathrm{C}(4)-\mathrm{C}(5)-\mathrm{N}(1)$ & $177.78(11)$ \\
\hline $\mathrm{C}(3)-\mathrm{C}(4)-\mathrm{C}(5)-\mathrm{N}(1)$ & $0.21(14)$ \\
\hline $\mathrm{C}(3)-\mathrm{C}(4)-\mathrm{C}(5)-\mathrm{C}(6)$ & $177.78(14)$ \\
\hline $\mathrm{N}(1)-\mathrm{C}(5)-\mathrm{C}(6)-\mathrm{O}(8)$ & $-178.95(11)$ \\
\hline $\mathrm{C}(4)-\mathrm{C}(5)-\mathrm{C}(6)-\mathrm{O}(8)$ & $3.7(2)$ \\
\hline $\mathrm{C}(4)-\mathrm{C}(5)-\mathrm{C}(6)-\mathrm{O}(7)$ & $-175.65(14)$ \\
\hline $\mathrm{N}(1)-\mathrm{C}(5)-\mathrm{C}(6)-\mathrm{O}(7)$ & $1.68(19)$ \\
\hline
\end{tabular}

Table 7. Hydrogen-bonds for compound 5 [A and deg.].

\begin{tabular}{c|c|c|c|c}
$\mathrm{D}-\mathrm{H} \ldots \mathrm{A}$ & $\mathrm{d}(\mathrm{D}-\mathrm{H})$ & $\mathrm{d}(\mathrm{H} \ldots \mathrm{A})$ & $\mathrm{d}(\mathrm{D} \ldots \mathrm{A})$ & $<(\mathrm{DHA})$ \\
\hline & & & & \\
\hline $\mathrm{N}(1)-\mathrm{H}(1) \ldots \mathrm{O}(7) \# 1$ & $0.867(18)$ & $2.032(17)$ & $2.8752(15)$ & $164.0(13)$ \\
\hline $\mathrm{N}(15)-\mathrm{H}(15) \ldots \mathrm{O}(17) \# 2$ & $0.827(18)$ & $2.112(18)$ & $2.9247(14)$ & $167.5(15)$ \\
\hline $\mathrm{C}(14)-\mathrm{H}(14 \mathrm{~A}) \ldots \mathrm{O}(17)$ & $0.979(15)$ & $2.373(15)$ & $2.8211(17)$ & $107.2(10)$ \\
\hline $\mathrm{C}(21)-\mathrm{H}(21 \mathrm{~A}) \ldots \mathrm{O}(17)$ & $0.956(19)$ & $2.456(18)$ & $2.9735(19)$ & $113.8(14)$ \\
\hline $\mathrm{C}(22)-\mathrm{H}(22 \mathrm{~A}) \ldots \mathrm{O}(17)$ & $0.971(19)$ & $2.468(19)$ & $2.994(2)$ & $113.8(14)$
\end{tabular}

Symmetry transformations used to generate equivalent atoms: \#1 -x+2,-y,-z \#2 1/2-x+1,1/2+y-1,1/2-z 


\section{Ac-MOPAS-D-Pro-Gly-Val-OMe, 10.}
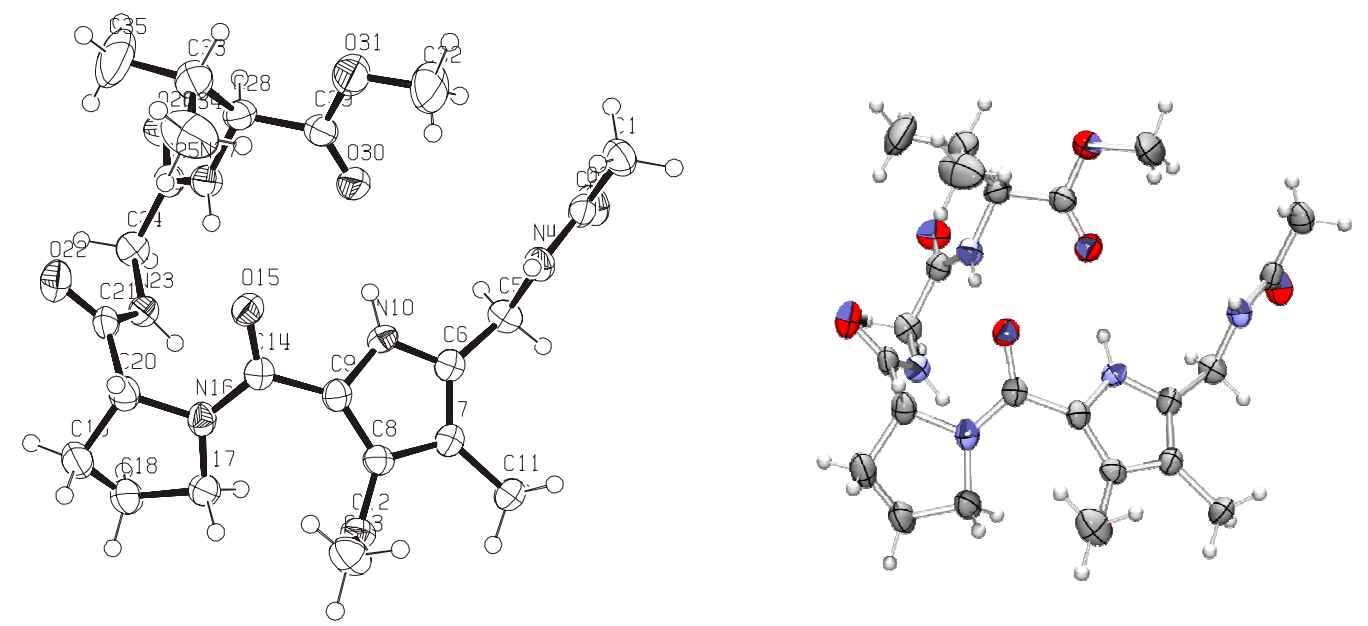

Table 1. Crystal data and structure refinement for compound $\mathbf{1 0 .}$

\begin{tabular}{|c|c|}
\hline Crystal Data & \\
\hline Empirical formula & C23 H35 N5 O7 \\
\hline Formula weight & 493.56 \\
\hline Crystal size & $0.55 \times 0.18 \times 0.12 \mathrm{~mm}$ \\
\hline Crystal description & thin rod \\
\hline Crystal colour & colourless \\
\hline Crystal system & Orthorhombic \\
\hline Space group & P 212121 \\
\hline \multirow[t]{3}{*}{ Unit cell dimensions } & $\mathrm{a}=8.7320(5) \mathrm{A} \quad$ alpha $=90 \mathrm{deg}$ \\
\hline & $\mathrm{b}=13.9621(13) \mathrm{A} \quad$ beta $=90 \mathrm{deg}$. \\
\hline & $\mathrm{c}=21.7439(14) \mathrm{A}$ gamma $=90 \mathrm{deg}$. \\
\hline Volume & $2651.0(3) \mathrm{A}^{\wedge} 3$ \\
\hline Z, Calculated density & $4,1.237 \mathrm{Mg} / \mathrm{m}^{\wedge} 3$ \\
\hline Absorption coefficient & $0.092 \mathrm{~mm}^{\wedge}-1$ \\
\hline $\mathrm{F}(000)$ & 1056 \\
\hline \multicolumn{2}{|l|}{ Data Collection } \\
\hline Measurement device type & STOE-IPDS diffractometer \\
\hline Measuremnet method & rotation \\
\hline Temperature & $173(1) \mathrm{K}$ \\
\hline Wavelength & $0.71073 \mathrm{~A}$ \\
\hline Monochromator & graphite \\
\hline Theta range for data collection & 2.37 to $25.81 \mathrm{deg}$. \\
\hline
\end{tabular}




\begin{tabular}{l|l} 
Index ranges & $-10<=\mathrm{h}<=10,-17<=\mathrm{k}<=17,-26<=\mathrm{l}<=26$ \\
\hline Reflections collected / unique & $37419 / 5078[\mathrm{R}(\mathrm{int})=0.1506]$ \\
\hline Reflections greater I $>2 \mathrm{lls}(\mathrm{I})$ & 4554 \\
\hline Absorption correction & None \\
\hline Refinement & \\
\hline Refinement method & Full-matrix least-squares on $\mathrm{F}^{\wedge} 2$ \\
\hline Hydrogen treatment & $:$ \\
\hline Data / restraints / parameters & $5078 / 0 / 316$ \\
\hline Goodness-of-fit on F^2 & 1.110 \\
\hline Final R indices [I $>2$ sigma(I)] & $\mathrm{R} 1=0.0743, \mathrm{wR} 2=0.1988$ \\
\hline R indices (all data) & $\mathrm{R} 1=0.0794, \mathrm{wR} 2=0.2022$ \\
\hline Absolute structure parameter & $0.3(19)$ \\
\hline Largest diff. peak and hole & 0.438 and -0.308 e. $\mathrm{A}^{\wedge}-3$ \\
\hline
\end{tabular}

Table 2. Atomic coordinates ( $\mathrm{x} 10^{\wedge} 4$ ) and equivalent isotropic displacement parameters $\left(\mathrm{A}^{\wedge} 2 \times 10^{\wedge} 3\right)$ for compound $\mathbf{1 0}$. $\mathrm{U}(\mathrm{eq})$ is defined as one third of the trace of the orthogonalized Uij tensor.

\begin{tabular}{|c|c|c|c|c|}
\hline & $\mathrm{X}$ & $\mathrm{y}$ & $\mathrm{Z}$ & $\mathrm{U}(\mathrm{eq})$ \\
\hline $\mathrm{O}(3)$ & 9091(3) & $9021(2)$ & $782(2)$ & $47(1)$ \\
\hline $\mathrm{O}(12)$ & $8711(3)$ & $4587(2)$ & $-1353(1)$ & $39(1)$ \\
\hline $\mathrm{O}(15)$ & $6088(3)$ & 4232(2) & 414(1) & $35(1)$ \\
\hline $\mathrm{O}(22)$ & 2444(3) & $3064(2)$ & $456(2)$ & $46(1)$ \\
\hline $\mathrm{O}(26)$ & 1198(4) & $5854(2)$ & 1191(2) & 49(1) \\
\hline $\mathrm{O}(30)$ & $6187(3)$ & $6173(2)$ & $1175(1)$ & $43(1)$ \\
\hline $\mathrm{O}(31)$ & $6308(5)$ & $5967(4)$ & $2179(2)$ & $78(2)$ \\
\hline $\mathrm{N}(4)$ & $9560(4)$ & $7458(2)$ & $596(2)$ & $35(1)$ \\
\hline $\mathrm{N}(10)$ & $7554(3)$ & $5834(2)$ & $-5(1)$ & $30(1)$ \\
\hline $\mathrm{N}(16)$ & $5538(3)$ & $3767(2)$ & $-555(2)$ & $33(1)$ \\
\hline $\mathrm{N}(23)$ & $2775(3)$ & $4449(2)$ & $-68(2)$ & $33(1)$ \\
\hline $\mathrm{N}(27)$ & $3531(4)$ & $5153(2)$ & $1088(2)$ & $36(1)$ \\
\hline $\mathrm{C}(1)$ & $10573(5)$ & $8172(3)$ & $1533(2)$ & $44(1)$ \\
\hline $\mathrm{C}(2)$ & 9664(4) & $8253(3)$ & $944(2)$ & $37(1)$ \\
\hline$C(5)$ & $8747(5)$ & $7472(3)$ & $10(2)$ & $37(1)$ \\
\hline $\mathrm{C}(6)$ & $8537(4)$ & 6489(3) & $-264(2)$ & $33(1)$ \\
\hline $\mathrm{C}(7)$ & $9108(4)$ & $6096(3)$ & $-796(2)$ & $33(1)$ \\
\hline $\mathrm{C}(8)$ & $8416(4)$ & $5178(3)$ & $-859(2)$ & 31(1) \\
\hline $\mathrm{C}(9)$ & $7445(4)$ & $5031(3)$ & $-359(2)$ & $32(1)$ \\
\hline
\end{tabular}




\begin{tabular}{l|c|c|c|c}
$\mathrm{C}(11)$ & $10246(5)$ & $6517(3)$ & $-1245(2)$ & $43(1)$ \\
\hline $\mathrm{C}(13)$ & $9614(5)$ & $3763(3)$ & $-1201(2)$ & $49(1)$ \\
\hline $\mathrm{C}(14)$ & $6334(4)$ & $4300(2)$ & $-145(2)$ & $30(1)$ \\
\hline $\mathrm{C}(17)$ & $5565(4)$ & $3802(3)$ & $-1226(2)$ & $40(1)$ \\
\hline $\mathrm{C}(18)$ & $4040(5)$ & $3326(4)$ & $-1397(2)$ & $49(1)$ \\
\hline $\mathrm{C}(19)$ & $3817(5)$ & $2580(3)$ & $-899(2)$ & $48(1)$ \\
\hline $\mathrm{C}(20)$ & $4413(4)$ & $3068(3)$ & $-312(2)$ & $37(1)$ \\
\hline $\mathrm{C}(21)$ & $3129(4)$ & $3539(3)$ & $61(2)$ & $34(1)$ \\
\hline $\mathrm{C}(24)$ & $1621(4)$ & $4976(3)$ & $270(2)$ & $38(1)$ \\
\hline $\mathrm{C}(25)$ & $2106(4)$ & $5369(3)$ & $894(2)$ & $37(1)$ \\
\hline $\mathrm{C}(28)$ & $4080(5)$ & $5410(3)$ & $1696(2)$ & $37(1)$ \\
\hline $\mathrm{C}(29)$ & $5649(5)$ & $5887(3)$ & $1640(2)$ & $37(1)$ \\
\hline $\mathrm{C}(32)$ & $7848(8)$ & $6354(8)$ & $2161(3)$ & $106(3)$ \\
\hline $\mathrm{C}(33)$ & $4098(6)$ & $4534(3)$ & $2142(2)$ & $52(1)$ \\
\hline $\mathrm{C}(34)$ & $5170(10)$ & $3760(4)$ & $1918(3)$ & $82(2)$ \\
\hline $\mathrm{C}(35)$ & $2446(9)$ & $4169(7)$ & $2224(3)$ & $102(3)$
\end{tabular}

Table 3. Bond lengths $[\mathrm{A}]$ and angles [deg] for compound $\mathbf{1 0 .}$

\begin{tabular}{c|c}
$\mathrm{O}(3)-\mathrm{C}(2)$ & $1.235(5)$ \\
\hline $\mathrm{O}(12)-\mathrm{C}(8)$ & $1.378(5)$ \\
\hline $\mathrm{O}(12)-\mathrm{C}(13)$ & $1.434(5)$ \\
\hline $\mathrm{O}(15)-\mathrm{C}(14)$ & $1.237(4)$ \\
\hline $\mathrm{O}(22)-\mathrm{C}(21)$ & $1.238(5)$ \\
\hline $\mathrm{O}(26)-\mathrm{C}(25)$ & $1.227(5)$ \\
\hline $\mathrm{O}(30)-\mathrm{C}(29)$ & $1.183(5)$ \\
\hline $\mathrm{O}(31)-\mathrm{C}(29)$ & $1.311(5)$ \\
\hline $\mathrm{O}(31)-\mathrm{C}(32)$ & $1.450(9)$ \\
\hline $\mathrm{N}(4)-\mathrm{C}(2)$ & $1.346(5)$ \\
\hline $\mathrm{N}(4)-\mathrm{C}(5)$ & $1.459(5)$ \\
\hline $\mathrm{N}(10)-\mathrm{C}(6)$ & $1.375(5)$ \\
\hline $\mathrm{N}(10)-\mathrm{C}(9)$ & $1.363(5)$ \\
\hline $\mathrm{N}(16)-\mathrm{C}(14)$ & $1.354(4)$ \\
\hline $\mathrm{N}(16)-\mathrm{C}(17)$ & $1.460(5)$ \\
\hline $\mathrm{N}(16)-\mathrm{C}(20)$ & $1.482(5)$ \\
\hline $\mathrm{N}(23)-\mathrm{C}(21)$ & $1.337(5)$ \\
\hline $\mathrm{N}(23)-\mathrm{C}(24)$ & $1.448(5)$ \\
\hline $\mathrm{N}(27)-\mathrm{C}(25)$ & $1.348(5)$ \\
\hline $\mathrm{N}(27)-\mathrm{C}(28)$ & $1.452(5)$ \\
\hline $\mathrm{N}(4)-\mathrm{H}(4)$ & 0.8803 \\
&
\end{tabular}




\begin{tabular}{|c|c|}
\hline $\mathrm{N}(10)-\mathrm{H}(10)$ & 0.8802 \\
\hline $\mathrm{N}(23)-\mathrm{H}(23)$ & 0.8796 \\
\hline $\mathrm{N}(27)-\mathrm{H}(27)$ & 0.8803 \\
\hline $\mathrm{C}(1)-\mathrm{C}(2)$ & $1.511(6)$ \\
\hline$C(5)-C(6)$ & $1.508(6)$ \\
\hline $\mathrm{C}(6)-\mathrm{C}(7)$ & $1.375(5)$ \\
\hline$C(7)-C(11)$ & $1.512(6)$ \\
\hline$C(7)-C(8)$ & $1.424(6)$ \\
\hline $\mathrm{C}(8)-\mathrm{C}(9)$ & $1.394(5)$ \\
\hline $\mathrm{C}(9)-\mathrm{C}(14)$ & $1.483(5)$ \\
\hline $\mathrm{C}(17)-\mathrm{C}(18)$ & $1.534(6)$ \\
\hline $\mathrm{C}(18)-\mathrm{C}(19)$ & $1.515(7)$ \\
\hline $\mathrm{C}(19)-\mathrm{C}(20)$ & $1.538(6)$ \\
\hline $\mathrm{C}(20)-\mathrm{C}(21)$ & $1.532(5)$ \\
\hline $\mathrm{C}(24)-\mathrm{C}(25)$ & $1.524(6)$ \\
\hline $\mathrm{C}(28)-\mathrm{C}(33)$ & $1.562(6)$ \\
\hline $\mathrm{C}(28)-\mathrm{C}(29)$ & $1.528(6)$ \\
\hline $\mathrm{C}(33)-\mathrm{C}(34)$ & $1.510(8)$ \\
\hline $\mathrm{C}(33)-\mathrm{C}(35)$ & $1.540(10)$ \\
\hline $\mathrm{C}(1)-\mathrm{H}(1 \mathrm{~A})$ & 0.9801 \\
\hline $\mathrm{C}(1)-\mathrm{H}(1 \mathrm{~B})$ & 0.9809 \\
\hline $\mathrm{C}(1)-\mathrm{H}(1 \mathrm{C})$ & 0.9792 \\
\hline $\mathrm{C}(5)-\mathrm{H}(5 \mathrm{~A})$ & 0.9904 \\
\hline $\mathrm{C}(5)-\mathrm{H}(5 \mathrm{~B})$ & 0.9898 \\
\hline $\mathrm{C}(11)-\mathrm{H}(11 \mathrm{~A})$ & 0.9799 \\
\hline $\mathrm{C}(11)-\mathrm{H}(11 \mathrm{~B})$ & 0.9806 \\
\hline $\mathrm{C}(11)-\mathrm{H}(11 \mathrm{C})$ & 0.9801 \\
\hline $\mathrm{C}(13)-\mathrm{H}(13 \mathrm{~A})$ & 0.9797 \\
\hline $\mathrm{C}(13)-\mathrm{H}(13 \mathrm{~B})$ & 0.9792 \\
\hline $\mathrm{C}(13)-\mathrm{H}(13 \mathrm{C})$ & 0.9809 \\
\hline $\mathrm{C}(17)-\mathrm{H}(17 \mathrm{~A})$ & 0.9899 \\
\hline $\mathrm{C}(17)-\mathrm{H}(17 \mathrm{~B})$ & 0.9892 \\
\hline $\mathrm{C}(18)-\mathrm{H}(18 \mathrm{~A})$ & 0.9900 \\
\hline $\mathrm{C}(18)-\mathrm{H}(18 \mathrm{~B})$ & 0.9895 \\
\hline $\mathrm{C}(19)-\mathrm{H}(19 \mathrm{~A})$ & 0.9903 \\
\hline $\mathrm{C}(19)-\mathrm{H}(19 \mathrm{~B})$ & 0.9901 \\
\hline $\mathrm{C}(20)-\mathrm{H}(20)$ & 1.0003 \\
\hline $\mathrm{C}(24)-\mathrm{H}(24 \mathrm{~A})$ & 0.9892 \\
\hline $\mathrm{C}(24)-\mathrm{H}(24 \mathrm{~B})$ & 0.9904 \\
\hline $\mathrm{C}(28)-\mathrm{H}(28)$ & 1.0002 \\
\hline
\end{tabular}




\begin{tabular}{l|l}
$\mathrm{C}(32)-\mathrm{H}(32 \mathrm{~A})$ & 0.9796 \\
\hline $\mathrm{C}(32)-\mathrm{H}(32 \mathrm{~B})$ & 0.9790 \\
\hline $\mathrm{C}(32)-\mathrm{H}(32 \mathrm{C})$ & 0.9808 \\
\hline $\mathrm{C}(33)-\mathrm{H}(33)$ & 0.9991 \\
\hline $\mathrm{C}(34)-\mathrm{H}(34 \mathrm{~A})$ & 0.9794 \\
\hline $\mathrm{C}(34)-\mathrm{H}(34 \mathrm{~B})$ & 0.9797 \\
\hline $\mathrm{C}(34)-\mathrm{H}(34 \mathrm{C})$ & 0.9806 \\
\hline $\mathrm{C}(35)-\mathrm{H}(35 \mathrm{~A})$ & 0.9804 \\
\hline $\mathrm{C}(35)-\mathrm{H}(35 \mathrm{~B})$ & 0.9800 \\
\hline $\mathrm{C}(35)-\mathrm{H}(35 \mathrm{C})$ & 0.9808
\end{tabular}

\begin{tabular}{c|c}
$\mathrm{C}(8)-\mathrm{O}(12)-\mathrm{C}(13)$ & $113.8(3)$ \\
\hline $\mathrm{C}(29)-\mathrm{O}(31)-\mathrm{C}(32)$ & $114.5(4)$ \\
\hline $\mathrm{C}(2)-\mathrm{N}(4)-\mathrm{C}(5)$ & $120.8(3)$ \\
\hline $\mathrm{C}(6)-\mathrm{N}(10)-\mathrm{C}(9)$ & $111.1(3)$ \\
\hline $\mathrm{C}(14)-\mathrm{N}(16)-\mathrm{C}(17)$ & $129.2(3)$ \\
\hline $\mathrm{C}(14)-\mathrm{N}(16)-\mathrm{C}(20)$ & $117.9(3)$ \\
\hline $\mathrm{C}(17)-\mathrm{N}(16)-\mathrm{C}(20)$ & $112.9(3)$ \\
\hline $\mathrm{C}(21)-\mathrm{N}(23)-\mathrm{C}(24)$ & $122.5(3)$ \\
\hline $\mathrm{C}(25)-\mathrm{N}(27)-\mathrm{C}(28)$ & $122.3(3)$ \\
\hline $\mathrm{C}(2)-\mathrm{N}(4)-\mathrm{H}(4)$ & 119.59 \\
\hline $\mathrm{C}(5)-\mathrm{N}(4)-\mathrm{H}(4)$ & 119.59 \\
\hline $\mathrm{C}(6)-\mathrm{N}(10)-\mathrm{H}(10)$ & 124.43 \\
\hline $\mathrm{C}(9)-\mathrm{N}(10)-\mathrm{H}(10)$ & 124.49 \\
\hline $\mathrm{C}(21)-\mathrm{N}(23)-\mathrm{H}(23)$ & 118.80 \\
\hline $\mathrm{C}(24)-\mathrm{N}(23)-\mathrm{H}(23)$ & 118.69 \\
\hline $\mathrm{C}(28)-\mathrm{N}(27)-\mathrm{H}(27)$ & 118.82 \\
\hline $\mathrm{C}(25)-\mathrm{N}(27)-\mathrm{H}(27)$ & 118.90 \\
\hline $\mathrm{O}(3)-\mathrm{C}(2)-\mathrm{N}(4)$ & $121.9(4)$ \\
\hline $\mathrm{O}(3)-\mathrm{C}(2)-\mathrm{C}(1)$ & $121.3(4)$ \\
\hline $\mathrm{N}(4)-\mathrm{C}(2)-\mathrm{C}(1)$ & $116.7(3)$ \\
\hline $\mathrm{N}(4)-\mathrm{C}(5)-\mathrm{C}(6)$ & $113.1(3)$ \\
\hline $\mathrm{N}(10)-\mathrm{C}(6)-\mathrm{C}(7)$ & $107.8(3)$ \\
\hline $\mathrm{C}(5)-\mathrm{C}(6)-\mathrm{C}(7)$ & $130.7(4)$ \\
\hline $\mathrm{N}(10)-\mathrm{C}(6)-\mathrm{C}(5)$ & $121.3(3)$ \\
\hline $\mathrm{C}(6)-\mathrm{C}(7)-\mathrm{C}(11)$ & $128.8(4)$ \\
\hline $\mathrm{C}(8)-\mathrm{C}(7)-\mathrm{C}(11)$ & $124.5(3)$ \\
\hline $\mathrm{C}(6)-\mathrm{C}(7)-\mathrm{C}(8)$ & $106.6(3)$ \\
\hline $\mathrm{C}(7)-\mathrm{C}(8)-\mathrm{C}(9)$ & $108.4(3)$ \\
\hline $\mathrm{O}(12)-\mathrm{C}(8)-\mathrm{C}(7)$ & $122.3(3)$ \\
\end{tabular}




\begin{tabular}{|c|c|}
\hline $\mathrm{O}(12)-\mathrm{C}(8)-\mathrm{C}(9)$ & $129.3(4)$ \\
\hline $\mathrm{C}(8)-\mathrm{C}(9)-\mathrm{C}(14)$ & $138.1(4)$ \\
\hline $\mathrm{N}(10)-\mathrm{C}(9)-\mathrm{C}(8)$ & 106.1(3) \\
\hline $\mathrm{N}(10)-\mathrm{C}(9)-\mathrm{C}(14)$ & 115.7(3) \\
\hline $\mathrm{N}(16)-\mathrm{C}(14)-\mathrm{C}(9)$ & $120.5(3)$ \\
\hline $\mathrm{O}(15)-\mathrm{C}(14)-\mathrm{N}(16)$ & $121.0(3)$ \\
\hline $\mathrm{O}(15)-\mathrm{C}(14)-\mathrm{C}(9)$ & $118.4(3)$ \\
\hline $\mathrm{N}(16)-\mathrm{C}(17)-\mathrm{C}(18)$ & $102.4(3)$ \\
\hline $\mathrm{C}(17)-\mathrm{C}(18)-\mathrm{C}(19)$ & $103.6(3)$ \\
\hline $\mathrm{C}(18)-\mathrm{C}(19)-\mathrm{C}(20)$ & $104.2(3)$ \\
\hline $\mathrm{N}(16)-\mathrm{C}(20)-\mathrm{C}(19)$ & $102.7(3)$ \\
\hline $\mathrm{C}(19)-\mathrm{C}(20)-\mathrm{C}(21)$ & $112.5(3)$ \\
\hline $\mathrm{N}(16)-\mathrm{C}(20)-\mathrm{C}(21)$ & $113.0(3)$ \\
\hline $\mathrm{O}(22)-\mathrm{C}(21)-\mathrm{N}(23)$ & $122.9(3)$ \\
\hline $\mathrm{O}(22)-\mathrm{C}(21)-\mathrm{C}(20)$ & $119.4(4)$ \\
\hline $\mathrm{N}(23)-\mathrm{C}(21)-\mathrm{C}(20)$ & $117.8(3)$ \\
\hline $\mathrm{N}(23)-\mathrm{C}(24)-\mathrm{C}(25)$ & $116.2(3)$ \\
\hline $\mathrm{O}(26)-\mathrm{C}(25)-\mathrm{N}(27)$ & $123.7(4)$ \\
\hline $\mathrm{O}(26)-\mathrm{C}(25)-\mathrm{C}(24)$ & $119.2(3)$ \\
\hline $\mathrm{N}(27)-\mathrm{C}(25)-\mathrm{C}(24)$ & $117.0(3)$ \\
\hline $\mathrm{N}(27)-\mathrm{C}(28)-\mathrm{C}(29)$ & $109.4(3)$ \\
\hline $\mathrm{C}(29)-\mathrm{C}(28)-\mathrm{C}(33)$ & $112.4(4)$ \\
\hline $\mathrm{N}(27)-\mathrm{C}(28)-\mathrm{C}(33)$ & $112.1(3)$ \\
\hline $\mathrm{O}(30)-\mathrm{C}(29)-\mathrm{C}(28)$ & $124.8(4)$ \\
\hline $\mathrm{O}(31)-\mathrm{C}(29)-\mathrm{C}(28)$ & $111.1(4)$ \\
\hline $\mathrm{O}(30)-\mathrm{C}(29)-\mathrm{O}(31)$ & $124.1(4)$ \\
\hline $\mathrm{C}(28)-\mathrm{C}(33)-\mathrm{C}(35)$ & $108.8(5)$ \\
\hline $\mathrm{C}(34)-\mathrm{C}(33)-\mathrm{C}(35)$ & $112.4(5)$ \\
\hline $\mathrm{C}(28)-\mathrm{C}(33)-\mathrm{C}(34)$ & $111.5(4)$ \\
\hline $\mathrm{C}(2)-\mathrm{C}(1)-\mathrm{H}(1 \mathrm{~A})$ & 109.48 \\
\hline $\mathrm{C}(2)-\mathrm{C}(1)-\mathrm{H}(1 \mathrm{~B})$ & 109.47 \\
\hline $\mathrm{C}(2)-\mathrm{C}(1)-\mathrm{H}(1 \mathrm{C})$ & 109.47 \\
\hline $\mathrm{H}(1 \mathrm{~A})-\mathrm{C}(1)-\mathrm{H}(1 \mathrm{~B})$ & 109.49 \\
\hline $\mathrm{H}(1 \mathrm{~A})-\mathrm{C}(1)-\mathrm{H}(1 \mathrm{C})$ & 109.44 \\
\hline $\mathrm{H}(1 \mathrm{~B})-\mathrm{C}(1)-\mathrm{H}(1 \mathrm{C})$ & 109.48 \\
\hline $\mathrm{N}(4)-\mathrm{C}(5)-\mathrm{H}(5 \mathrm{~A})$ & 108.91 \\
\hline $\mathrm{N}(4)-\mathrm{C}(5)-\mathrm{H}(5 \mathrm{~B})$ & 109.01 \\
\hline $\mathrm{C}(6)-\mathrm{C}(5)-\mathrm{H}(5 \mathrm{~A})$ & 108.97 \\
\hline $\mathrm{C}(6)-\mathrm{C}(5)-\mathrm{H}(5 \mathrm{~B})$ & 108.97 \\
\hline $\mathrm{H}(5 \mathrm{~A})-\mathrm{C}(5)-\mathrm{H}(5 \mathrm{~B})$ & 107.74 \\
\hline
\end{tabular}




\begin{tabular}{|c|c|}
\hline $\mathrm{C}(7)-\mathrm{C}(11)-\mathrm{H}(11 \mathrm{~A})$ & 109.45 \\
\hline $\mathrm{C}(7)-\mathrm{C}(11)-\mathrm{H}(11 \mathrm{~B})$ & 109.45 \\
\hline $\mathrm{C}(7)-\mathrm{C}(11)-\mathrm{H}(11 \mathrm{C})$ & 109.43 \\
\hline $\mathrm{H}(11 \mathrm{~A})-\mathrm{C}(11)-\mathrm{H}(11 \mathrm{~B})$ & 109.48 \\
\hline $\mathrm{H}(11 \mathrm{~A})-\mathrm{C}(11)-\mathrm{H}(11 \mathrm{C})$ & 109.53 \\
\hline $\mathrm{H}(11 \mathrm{~B})-\mathrm{C}(11)-\mathrm{H}(11 \mathrm{C})$ & 109.50 \\
\hline $\mathrm{O}(12)-\mathrm{C}(13)-\mathrm{H}(13 \mathrm{~A})$ & 109.48 \\
\hline $\mathrm{O}(12)-\mathrm{C}(13)-\mathrm{H}(13 \mathrm{~B})$ & 109.53 \\
\hline $\mathrm{O}(12)-\mathrm{C}(13)-\mathrm{H}(13 \mathrm{C})$ & 109.42 \\
\hline $\mathrm{H}(13 \mathrm{~A})-\mathrm{C}(13)-\mathrm{H}(13 \mathrm{~B})$ & 109.61 \\
\hline $\mathrm{H}(13 \mathrm{~A})-\mathrm{C}(13)-\mathrm{H}(13 \mathrm{C})$ & 109.38 \\
\hline $\mathrm{H}(13 \mathrm{~B})-\mathrm{C}(13)-\mathrm{H}(13 \mathrm{C})$ & 109.41 \\
\hline $\mathrm{N}(16)-\mathrm{C}(17)-\mathrm{H}(17 \mathrm{~A})$ & 111.28 \\
\hline $\mathrm{N}(16)-\mathrm{C}(17)-\mathrm{H}(17 \mathrm{~B})$ & 111.26 \\
\hline $\mathrm{C}(18)-\mathrm{C}(17)-\mathrm{H}(17 \mathrm{~A})$ & 111.26 \\
\hline $\mathrm{C}(18)-\mathrm{C}(17)-\mathrm{H}(17 \mathrm{~B})$ & 111.31 \\
\hline $\mathrm{H}(17 \mathrm{~A})-\mathrm{C}(17)-\mathrm{H}(17 \mathrm{~B})$ & 109.25 \\
\hline $\mathrm{C}(17)-\mathrm{C}(18)-\mathrm{H}(18 \mathrm{~A})$ & 110.99 \\
\hline $\mathrm{C}(17)-\mathrm{C}(18)-\mathrm{H}(18 \mathrm{~B})$ & 111.03 \\
\hline $\mathrm{C}(19)-\mathrm{C}(18)-\mathrm{H}(18 \mathrm{~A})$ & 111.01 \\
\hline $\mathrm{C}(19)-\mathrm{C}(18)-\mathrm{H}(18 \mathrm{~B})$ & 111.05 \\
\hline $\mathrm{H}(18 \mathrm{~A})-\mathrm{C}(18)-\mathrm{H}(18 \mathrm{~B})$ & 109.06 \\
\hline $\mathrm{C}(18)-\mathrm{C}(19)-\mathrm{H}(19 \mathrm{~A})$ & 110.93 \\
\hline $\mathrm{C}(18)-\mathrm{C}(19)-\mathrm{H}(19 \mathrm{~B})$ & 110.90 \\
\hline $\mathrm{C}(20)-\mathrm{C}(19)-\mathrm{H}(19 \mathrm{~A})$ & 110.92 \\
\hline $\mathrm{C}(20)-\mathrm{C}(19)-\mathrm{H}(19 \mathrm{~B})$ & 110.95 \\
\hline $\mathrm{H}(19 \mathrm{~A})-\mathrm{C}(19)-\mathrm{H}(19 \mathrm{~B})$ & 108.89 \\
\hline $\mathrm{N}(16)-\mathrm{C}(20)-\mathrm{H}(20)$ & 109.53 \\
\hline $\mathrm{C}(19)-\mathrm{C}(20)-\mathrm{H}(20)$ & 109.45 \\
\hline $\mathrm{C}(21)-\mathrm{C}(20)-\mathrm{H}(20)$ & 109.48 \\
\hline $\mathrm{N}(23)-\mathrm{C}(24)-\mathrm{H}(24 \mathrm{~A})$ & 108.25 \\
\hline $\mathrm{N}(23)-\mathrm{C}(24)-\mathrm{H}(24 \mathrm{~B})$ & 108.26 \\
\hline $\mathrm{C}(25)-\mathrm{C}(24)-\mathrm{H}(24 \mathrm{~A})$ & 108.26 \\
\hline $\mathrm{C}(25)-\mathrm{C}(24)-\mathrm{H}(24 \mathrm{~B})$ & 108.18 \\
\hline $\mathrm{H}(24 \mathrm{~A})-\mathrm{C}(24)-\mathrm{H}(24 \mathrm{~B})$ & 107.40 \\
\hline $\mathrm{N}(27)-\mathrm{C}(28)-\mathrm{H}(28)$ & 107.58 \\
\hline $\mathrm{C}(29)-\mathrm{C}(28)-\mathrm{H}(28)$ & 107.55 \\
\hline $\mathrm{C}(33)-\mathrm{C}(28)-\mathrm{H}(28)$ & 107.60 \\
\hline $\mathrm{O}(31)-\mathrm{C}(32)-\mathrm{H}(32 \mathrm{~A})$ & 109.47 \\
\hline $\mathrm{O}(31)-\mathrm{C}(32)-\mathrm{H}(32 \mathrm{~B})$ & 109.53 \\
\hline
\end{tabular}




\begin{tabular}{c|c}
$\mathrm{O}(31)-\mathrm{C}(32)-\mathrm{H}(32 \mathrm{C})$ & 109.42 \\
\hline $\mathrm{H}(32 \mathrm{~A})-\mathrm{C}(32)-\mathrm{H}(32 \mathrm{~B})$ & 109.59 \\
\hline $\mathrm{H}(32 \mathrm{~A})-\mathrm{C}(32)-\mathrm{H}(32 \mathrm{C})$ & 109.41 \\
\hline $\mathrm{H}(32 \mathrm{~B})-\mathrm{C}(32)-\mathrm{H}(32 \mathrm{C})$ & 109.40 \\
\hline $\mathrm{C}(28)-\mathrm{C}(33)-\mathrm{H}(33)$ & 108.01 \\
\hline $\mathrm{C}(34)-\mathrm{C}(33)-\mathrm{H}(33)$ & 108.02 \\
\hline $\mathrm{C}(35)-\mathrm{C}(33)-\mathrm{H}(33)$ & 108.01 \\
\hline $\mathrm{C}(33)-\mathrm{C}(34)-\mathrm{H}(34 \mathrm{~A})$ & 109.45 \\
\hline $\mathrm{C}(33)-\mathrm{C}(34)-\mathrm{H}(34 \mathrm{~B})$ & 109.40 \\
\hline $\mathrm{C}(33)-\mathrm{C}(34)-\mathrm{H}(34 \mathrm{C})$ & 109.39 \\
\hline $\mathrm{H}(34 \mathrm{~A})-\mathrm{C}(34)-\mathrm{H}(34 \mathrm{~B})$ & 109.55 \\
\hline $\mathrm{H}(34 \mathrm{~A})-\mathrm{C}(34)-\mathrm{H}(34 \mathrm{C})$ & 109.54 \\
\hline $\mathrm{H}(34 \mathrm{~B})-\mathrm{C}(34)-\mathrm{H}(34 \mathrm{C})$ & 109.50 \\
\hline $\mathrm{C}(33)-\mathrm{C}(35)-\mathrm{H}(35 \mathrm{~A})$ & 109.50 \\
\hline $\mathrm{C}(33)-\mathrm{C}(35)-\mathrm{H}(35 \mathrm{~B})$ & 109.55 \\
\hline $\mathrm{C}(33)-\mathrm{C}(35)-\mathrm{H}(35 \mathrm{C})$ & 109.42 \\
\hline $\mathrm{H}(35 \mathrm{~A})-\mathrm{C}(35)-\mathrm{H}(35 \mathrm{~B})$ & 109.60 \\
\hline $\mathrm{H}(35 \mathrm{~A})-\mathrm{C}(35)-\mathrm{H}(35 \mathrm{C})$ & 109.34 \\
\hline $\mathrm{H}(35 \mathrm{~B})-\mathrm{C}(35)-\mathrm{H}(35 \mathrm{C})$ & 109.42 \\
\hline
\end{tabular}

Table 4. Anisotropic displacement parameters $\left(\mathrm{A}^{\wedge} 2 \times 10^{\wedge} 3\right)$ for compound $\mathbf{1 0}$. The anisotropic displacement factor exponent takes the form: $-2 \mathrm{pi}^{\wedge} 2\left[\mathrm{~h}^{\wedge} 2 \mathrm{a}^{* \wedge} 2 \mathrm{U} 11+\ldots+2 \mathrm{~h} \mathrm{k}\right.$ $a^{*} b * U 12$ ]

\begin{tabular}{c|c|c|c|c|c|c} 
& $\mathrm{U} 11$ & $\mathrm{U} 22$ & $\mathrm{U} 33$ & $\mathrm{U} 23$ & $\mathrm{U} 13$ & $\mathrm{U} 12$ \\
\hline $\mathrm{O}(3)$ & $48(2)$ & $28(1)$ & $66(2)$ & $-4(1)$ & $-3(2)$ & $0(1)$ \\
\hline $\mathrm{O}(12)$ & $44(2)$ & $35(1)$ & $37(1)$ & $-5(1)$ & $4(1)$ & $1(1)$ \\
\hline $\mathrm{O}(15)$ & $31(1)$ & $40(1)$ & $35(1)$ & $2(1)$ & $2(1)$ & $-1(1)$ \\
\hline $\mathrm{O}(22)$ & $40(2)$ & $37(1)$ & $63(2)$ & $5(1)$ & $4(1)$ & $-6(1)$ \\
\hline $\mathrm{O}(26)$ & $45(2)$ & $52(2)$ & $49(2)$ & $-3(1)$ & $3(1)$ & $15(1)$ \\
\hline $\mathrm{O}(30)$ & $50(2)$ & $38(1)$ & $41(2)$ & $-3(1)$ & $8(1)$ & $-7(1)$ \\
\hline $\mathrm{O}(31)$ & $70(2)$ & $125(4)$ & $37(2)$ & $8(2)$ & $-9(2)$ & $-48(3)$ \\
\hline $\mathrm{N}(4)$ & $36(2)$ & $27(1)$ & $41(2)$ & $-1(1)$ & $-1(1)$ & $-1(1)$ \\
\hline $\mathrm{N}(10)$ & $28(1)$ & $32(1)$ & $30(1)$ & $0(1)$ & $5(1)$ & $3(1)$ \\
\hline $\mathrm{N}(16)$ & $25(1)$ & $32(2)$ & $44(2)$ & $-2(1)$ & $0(1)$ & $-1(1)$ \\
\hline $\mathrm{N}(23)$ & $27(1)$ & $35(2)$ & $36(2)$ & $-1(1)$ & $0(1)$ & $4(1)$ \\
\hline $\mathrm{N}(27)$ & $29(2)$ & $42(2)$ & $36(2)$ & $-3(1)$ & $-2(1)$ & $2(1)$ \\
\hline $\mathrm{C}(1)$ & $44(2)$ & $39(2)$ & $49(2)$ & $-9(2)$ & $-2(2)$ & $-5(2)$ \\
\hline $\mathrm{C}(2)$ & $33(2)$ & $33(2)$ & $44(2)$ & $-2(2)$ & $8(2)$ & $-8(2)$ \\
& & & & & &
\end{tabular}




\begin{tabular}{c|c|c|c|c|c|c}
$\mathrm{C}(5)$ & $41(2)$ & $33(2)$ & $39(2)$ & $0(2)$ & $-3(2)$ & $5(2)$ \\
\hline $\mathrm{C}(6)$ & $31(2)$ & $33(2)$ & $35(2)$ & $2(1)$ & $-1(2)$ & $1(1)$ \\
\hline $\mathrm{C}(7)$ & $30(2)$ & $34(2)$ & $35(2)$ & $4(1)$ & $1(2)$ & $-2(1)$ \\
\hline $\mathrm{C}(8)$ & $27(2)$ & $36(2)$ & $29(2)$ & $-2(1)$ & $-4(1)$ & $3(1)$ \\
\hline $\mathrm{C}(9)$ & $25(2)$ & $35(2)$ & $35(2)$ & $0(1)$ & $-3(1)$ & $1(1)$ \\
\hline $\mathrm{C}(11)$ & $48(2)$ & $45(2)$ & $37(2)$ & $0(2)$ & $11(2)$ & $-15(2)$ \\
\hline $\mathrm{C}(13)$ & $43(2)$ & $46(2)$ & $59(3)$ & $-15(2)$ & $-7(2)$ & $12(2)$ \\
\hline $\mathrm{C}(14)$ & $26(2)$ & $29(2)$ & $36(2)$ & $2(1)$ & $-3(1)$ & $4(1)$ \\
\hline $\mathrm{C}(17)$ & $30(2)$ & $49(2)$ & $41(2)$ & $-10(2)$ & $2(2)$ & $-3(2)$ \\
\hline $\mathrm{C}(18)$ & $35(2)$ & $63(3)$ & $49(2)$ & $-15(2)$ & $-1(2)$ & $-11(2)$ \\
\hline $\mathrm{C}(19)$ & $39(2)$ & $45(2)$ & $61(3)$ & $-18(2)$ & $3(2)$ & $-7(2)$ \\
\hline $\mathrm{C}(20)$ & $34(2)$ & $31(2)$ & $46(2)$ & $-3(2)$ & $2(2)$ & $0(2)$ \\
\hline $\mathrm{C}(21)$ & $27(2)$ & $35(2)$ & $40(2)$ & $-5(2)$ & $-1(2)$ & $-8(1)$ \\
\hline $\mathrm{C}(24)$ & $30(2)$ & $39(2)$ & $45(2)$ & $-1(2)$ & $-4(2)$ & $8(2)$ \\
\hline $\mathrm{C}(25)$ & $37(2)$ & $32(2)$ & $41(2)$ & $1(2)$ & $2(2)$ & $1(2)$ \\
\hline $\mathrm{C}(28)$ & $43(2)$ & $39(2)$ & $31(2)$ & $1(2)$ & $4(2)$ & $-1(2)$ \\
\hline $\mathrm{C}(29)$ & $43(2)$ & $35(2)$ & $33(2)$ & $-2(2)$ & $-1(2)$ & $-1(2)$ \\
\hline $\mathrm{C}(32)$ & $81(5)$ & $184(8)$ & $52(3)$ & $13(4)$ & $-22(3)$ & $-75(5)$ \\
\hline $\mathrm{C}(33)$ & $65(3)$ & $51(2)$ & $41(2)$ & $12(2)$ & $-5(2)$ & $-11(2)$ \\
\hline $\mathrm{C}(34)$ & $147(6)$ & $43(3)$ & $55(3)$ & $11(2)$ & $-18(4)$ & $7(3)$ \\
\hline $\mathrm{C}(35)$ & $97(5)$ & $129(6)$ & $81(4)$ & $50(4)$ & $-6(4)$ & $-60(5)$ \\
& & & & & \\
\hline
\end{tabular}

Table 5. Hydrogen coordinates ( $\left.\mathrm{x} 10^{\wedge} 4\right)$ and isotropic displacement parameters $\left(\mathrm{A}^{\wedge} 2 \mathrm{x}\right.$ $10^{\wedge} 3$ ) for compound $\mathbf{1 0 .}$

\begin{tabular}{c|c|c|c|c} 
& $\mathrm{x}$ & $\mathrm{y}$ & $\mathrm{z}$ & $\mathrm{U}(\mathrm{eq})$ \\
\hline $\mathrm{H}(1 \mathrm{~A})$ & 11545 & 8516 & 1488 & 53 \\
\hline $\mathrm{H}(1 \mathrm{~B})$ & 10778 & 7495 & 1621 & 53 \\
\hline $\mathrm{H}(1 \mathrm{C})$ & 9986 & 8452 & 1872 & 53 \\
\hline $\mathrm{H}(4)$ & 9988 & 6923 & 724 & 42 \\
\hline $\mathrm{H}(5 \mathrm{~A})$ & 9324 & 7876 & -284 & 45 \\
\hline $\mathrm{H}(5 \mathrm{~B})$ & 7729 & 7769 & 71 & 45 \\
\hline $\mathrm{H}(10)$ & 7060 & 5922 & 343 & 36 \\
\hline $\mathrm{H}(11 \mathrm{~A})$ & 11215 & 6643 & -1033 & 52 \\
\hline $\mathrm{H}(11 \mathrm{~B})$ & 9839 & 7118 & -1412 & 52 \\
\hline $\mathrm{H}(11 \mathrm{C})$ & 10420 & 6063 & -1582 & 52 \\
\hline $\mathrm{H}(13 \mathrm{~A})$ & 9074 & 3378 & -893 & 59 \\
\hline $\mathrm{H}(13 \mathrm{~B})$ & 10604 & 3969 & -1036 & 59 \\
\hline $\mathrm{H}(13 \mathrm{C})$ & 9780 & 3377 & -1572 & 59 \\
\hline
\end{tabular}




\begin{tabular}{c|c|c|c|c}
$\mathrm{H}(17 \mathrm{~A})$ & 6445 & 3439 & -1393 & 48 \\
\hline $\mathrm{H}(17 \mathrm{~B})$ & 5611 & 4470 & -1376 & 48 \\
\hline $\mathrm{H}(18 \mathrm{~A})$ & 3194 & 3798 & -1395 & 59 \\
\hline $\mathrm{H}(18 \mathrm{~B})$ & 4099 & 3024 & -1808 & 59 \\
\hline $\mathrm{H}(19 \mathrm{~A})$ & 4411 & 1993 & -991 & 58 \\
\hline $\mathrm{H}(19 \mathrm{~B})$ & 2722 & 2408 & -857 & 58 \\
\hline $\mathrm{H}(20)$ & 4953 & 2585 & -50 & 44 \\
\hline $\mathrm{H}(23)$ & 3262 & 4739 & -369 & 39 \\
\hline $\mathrm{H}(24 \mathrm{~A})$ & 728 & 4551 & 332 & 46 \\
\hline $\mathrm{H}(24 \mathrm{~B})$ & 1278 & 5520 & 12 & 46 \\
\hline $\mathrm{H}(27)$ & 4150 & 4844 & 837 & 43 \\
\hline $\mathrm{H}(28)$ & 3356 & 5895 & 1869 & 45 \\
\hline $\mathrm{H}(32 \mathrm{~A})$ & 7824 & 6999 & 1984 & 127 \\
\hline $\mathrm{H}(32 \mathrm{~B})$ & 8500 & 5940 & 1909 & 127 \\
\hline $\mathrm{H}(32 \mathrm{C})$ & 8261 & 6385 & 2580 & 127 \\
\hline $\mathrm{H}(33)$ & 4474 & 4760 & 2551 & 62 \\
\hline $\mathrm{H}(34 \mathrm{~A})$ & 4841 & 3541 & 1511 & 98 \\
\hline $\mathrm{H}(34 \mathrm{~B})$ & 6212 & 4017 & 1892 & 98 \\
\hline $\mathrm{H}(34 \mathrm{C})$ & 5151 & 3221 & 2207 & 98 \\
\hline $\mathrm{H}(35 \mathrm{~A})$ & 1811 & 4680 & 2399 & 123 \\
\hline $\mathrm{H}(35 \mathrm{~B})$ & 2031 & 3976 & 1824 & 123 \\
\hline $\mathrm{H}(35 \mathrm{C})$ & 2445 & 3617 & 2503 & 123 \\
\hline
\end{tabular}

Table 6. Torsion angles [deg] for compound $\mathbf{1 0 .}$

\begin{tabular}{c|c}
$\mathrm{C}(13)-\mathrm{O}(12)-\mathrm{C}(8)-\mathrm{C}(7)$ & $109.0(4)$ \\
\hline $\mathrm{C}(13)-\mathrm{O}(12)-\mathrm{C}(8)-\mathrm{C}(9)$ & $-72.0(5)$ \\
\hline $\mathrm{C}(32)-\mathrm{O}(31)-\mathrm{C}(29)-\mathrm{O}(30)$ & $-6.0(8)$ \\
\hline $\mathrm{C}(32)-\mathrm{O}(31)-\mathrm{C}(29)-\mathrm{C}(28)$ & $176.0(6)$ \\
\hline $\mathrm{C}(5)-\mathrm{N}(4)-\mathrm{C}(2)-\mathrm{C}(1)$ & $-178.1(3)$ \\
\hline $\mathrm{C}(2)-\mathrm{N}(4)-\mathrm{C}(5)-\mathrm{C}(6)$ & $-170.9(3)$ \\
\hline $\mathrm{C}(5)-\mathrm{N}(4)-\mathrm{C}(2)-\mathrm{O}(3)$ & $-0.5(6)$ \\
\hline $\mathrm{C}(9)-\mathrm{N}(10)-\mathrm{C}(6)-\mathrm{C}(7)$ & $-1.1(4)$ \\
\hline $\mathrm{C}(9)-\mathrm{N}(10)-\mathrm{C}(6)-\mathrm{C}(5)$ & $174.6(3)$ \\
\hline $\mathrm{C}(6)-\mathrm{N}(10)-\mathrm{C}(9)-\mathrm{C}(8)$ & $0.8(4)$ \\
\hline $\mathrm{C}(6)-\mathrm{N}(10)-\mathrm{C}(9)-\mathrm{C}(14)$ & $-176.1(3)$ \\
\hline $\mathrm{C}(20)-\mathrm{N}(16)-\mathrm{C}(14)-\mathrm{C}(9)$ & $-178.3(3)$ \\
\hline $\mathrm{C}(17)-\mathrm{N}(16)-\mathrm{C}(14)-\mathrm{O}(15)$ & $174.2(3)$ \\
\hline $\mathrm{C}(17)-\mathrm{N}(16)-\mathrm{C}(20)-\mathrm{C}(19)$ & $5.2(4)$ \\
\hline $\mathrm{C}(14)-\mathrm{N}(16)-\mathrm{C}(17)-\mathrm{C}(18)$ & $-159.3(4)$ \\
\hline
\end{tabular}




\begin{tabular}{|c|c|}
\hline $\mathrm{C}(17)-\mathrm{N}(16)-\mathrm{C}(14)-\mathrm{C}(9)$ & $-1.4(5)$ \\
\hline $\mathrm{C}(17)-\mathrm{N}(16)-\mathrm{C}(20)-\mathrm{C}(21)$ & $-116.2(3)$ \\
\hline $\mathrm{C}(20)-\mathrm{N}(16)-\mathrm{C}(14)-\mathrm{O}(15)$ & $-2.7(5)$ \\
\hline $\mathrm{C}(20)-\mathrm{N}(16)-\mathrm{C}(17)-\mathrm{C}(18)$ & $17.7(4)$ \\
\hline $\mathrm{C}(14)-\mathrm{N}(16)-\mathrm{C}(20)-\mathrm{C}(19)$ & $-177.4(3)$ \\
\hline $\mathrm{C}(14)-\mathrm{N}(16)-\mathrm{C}(20)-\mathrm{C}(21)$ & $61.2(4)$ \\
\hline $\mathrm{C}(24)-\mathrm{N}(23)-\mathrm{C}(21)-\mathrm{C}(20)$ & $-177.7(3)$ \\
\hline $\mathrm{C}(21)-\mathrm{N}(23)-\mathrm{C}(24)-\mathrm{C}(25)$ & $78.9(4)$ \\
\hline $\mathrm{C}(24)-\mathrm{N}(23)-\mathrm{C}(21)-\mathrm{O}(22)$ & $3.9(6)$ \\
\hline $\mathrm{C}(25)-\mathrm{N}(27)-\mathrm{C}(28)-\mathrm{C}(33)$ & $103.1(4)$ \\
\hline $\mathrm{C}(28)-\mathrm{N}(27)-\mathrm{C}(25)-\mathrm{O}(26)$ & $5.4(6)$ \\
\hline $\mathrm{C}(28)-\mathrm{N}(27)-\mathrm{C}(25)-\mathrm{C}(24)$ & $-174.3(3)$ \\
\hline $\mathrm{C}(25)-\mathrm{N}(27)-\mathrm{C}(28)-\mathrm{C}(29)$ & $-131.5(4)$ \\
\hline $\mathrm{N}(4)-\mathrm{C}(5)-\mathrm{C}(6)-\mathrm{N}(10)$ & $70.2(5)$ \\
\hline $\mathrm{N}(4)-\mathrm{C}(5)-\mathrm{C}(6)-\mathrm{C}(7)$ & $-115.3(5)$ \\
\hline $\mathrm{N}(10)-\mathrm{C}(6)-\mathrm{C}(7)-\mathrm{C}(11)$ & $-179.1(4)$ \\
\hline $\mathrm{N}(10)-\mathrm{C}(6)-\mathrm{C}(7)-\mathrm{C}(8)$ & $0.9(4)$ \\
\hline$C(5)-C(6)-C(7)-C(11)$ & $5.8(7)$ \\
\hline $\mathrm{C}(5)-\mathrm{C}(6)-\mathrm{C}(7)-\mathrm{C}(8)$ & $-174.2(4)$ \\
\hline $\mathrm{C}(11)-\mathrm{C}(7)-\mathrm{C}(8)-\mathrm{C}(9)$ & $179.5(4)$ \\
\hline $\mathrm{C}(6)-\mathrm{C}(7)-\mathrm{C}(8)-\mathrm{O}(12)$ & $178.8(3)$ \\
\hline $\mathrm{C}(6)-\mathrm{C}(7)-\mathrm{C}(8)-\mathrm{C}(9)$ & $-0.4(4)$ \\
\hline $\mathrm{C}(11)-\mathrm{C}(7)-\mathrm{C}(8)-\mathrm{O}(12)$ & $-1.2(6)$ \\
\hline $\mathrm{O}(12)-\mathrm{C}(8)-\mathrm{C}(9)-\mathrm{N}(10)$ & $-179.4(3)$ \\
\hline $\mathrm{C}(7)-\mathrm{C}(8)-\mathrm{C}(9)-\mathrm{N}(10)$ & $-0.2(4)$ \\
\hline $\mathrm{C}(7)-\mathrm{C}(8)-\mathrm{C}(9)-\mathrm{C}(14)$ & $175.7(4)$ \\
\hline $\mathrm{O}(12)-\mathrm{C}(8)-\mathrm{C}(9)-\mathrm{C}(14)$ & $-3.5(7)$ \\
\hline $\mathrm{C}(8)-\mathrm{C}(9)-\mathrm{C}(14)-\mathrm{O}(15)$ & $154.2(4)$ \\
\hline $\mathrm{C}(8)-\mathrm{C}(9)-\mathrm{C}(14)-\mathrm{N}(16)$ & $-30.1(6)$ \\
\hline $\mathrm{N}(10)-\mathrm{C}(9)-\mathrm{C}(14)-\mathrm{N}(16)$ & $145.5(3)$ \\
\hline $\mathrm{N}(10)-\mathrm{C}(9)-\mathrm{C}(14)-\mathrm{O}(15)$ & $-30.2(5)$ \\
\hline $\mathrm{N}(16)-\mathrm{C}(17)-\mathrm{C}(18)-\mathrm{C}(19)$ & $-33.7(4)$ \\
\hline $\mathrm{C}(17)-\mathrm{C}(18)-\mathrm{C}(19)-\mathrm{C}(20)$ & $37.7(4)$ \\
\hline $\mathrm{C}(18)-\mathrm{C}(19)-\mathrm{C}(20)-\mathrm{C}(21)$ & $95.3(4)$ \\
\hline $\mathrm{C}(18)-\mathrm{C}(19)-\mathrm{C}(20)-\mathrm{N}(16)$ & $-26.5(4)$ \\
\hline $\mathrm{N}(16)-\mathrm{C}(20)-\mathrm{C}(21)-\mathrm{N}(23)$ & $26.4(5)$ \\
\hline $\mathrm{C}(19)-\mathrm{C}(20)-\mathrm{C}(21)-\mathrm{N}(23)$ & $-89.3(4)$ \\
\hline $\mathrm{N}(16)-\mathrm{C}(20)-\mathrm{C}(21)-\mathrm{O}(22)$ & $-155.2(3)$ \\
\hline $\mathrm{C}(19)-\mathrm{C}(20)-\mathrm{C}(21)-\mathrm{O}(22)$ & $89.1(4)$ \\
\hline $\mathrm{N}(23)-\mathrm{C}(24)-\mathrm{C}(25)-\mathrm{N}(27)$ & $-3.1(5)$ \\
\hline
\end{tabular}




\begin{tabular}{l|l}
$\mathrm{N}(23)-\mathrm{C}(24)-\mathrm{C}(25)-\mathrm{O}(26)$ & $177.2(4)$ \\
\hline $\mathrm{C}(29)-\mathrm{C}(28)-\mathrm{C}(33)-\mathrm{C}(34)$ & $-61.6(5)$ \\
\hline $\mathrm{N}(27)-\mathrm{C}(28)-\mathrm{C}(29)-\mathrm{O}(31)$ & $-168.2(4)$ \\
\hline $\mathrm{C}(33)-\mathrm{C}(28)-\mathrm{C}(29)-\mathrm{O}(30)$ & $139.0(4)$ \\
\hline $\mathrm{C}(33)-\mathrm{C}(28)-\mathrm{C}(29)-\mathrm{O}(31)$ & $-43.0(5)$ \\
\hline $\mathrm{N}(27)-\mathrm{C}(28)-\mathrm{C}(29)-\mathrm{O}(30)$ & $13.8(6)$ \\
\hline $\mathrm{C}(29)-\mathrm{C}(28)-\mathrm{C}(33)-\mathrm{C}(35)$ & $173.9(4)$ \\
\hline $\mathrm{N}(27)-\mathrm{C}(28)-\mathrm{C}(33)-\mathrm{C}(35)$ & $-62.4(5)$ \\
\hline $\mathrm{N}(27)-\mathrm{C}(28)-\mathrm{C}(33)-\mathrm{C}(34)$ & $62.1(6)$
\end{tabular}

Table 7. Hydrogen-bonds for compound $\mathbf{1 0}$ [A and deg.].

\begin{tabular}{c|c|c|c|c}
$\mathrm{D}-\mathrm{H} \ldots \mathrm{A}$ & $\mathrm{d}(\mathrm{D}-\mathrm{H})$ & $\mathrm{d}(\mathrm{H} \ldots \mathrm{A})$ & $\mathrm{d}(\mathrm{D} \ldots \mathrm{A})$ & $<(\mathrm{DHA})$ \\
\hline $\mathrm{N}(4)-\mathrm{H}(4) \ldots \mathrm{O}(26) \# 1$ & 0.8800 & 2.0900 & $2.956(4)$ & 167.00 \\
\hline $\mathrm{N}(10)-\mathrm{H}(10) \ldots \mathrm{O}(30)$ & 0.8800 & 1.9900 & $2.870(4)$ & 173.00 \\
\hline $\mathrm{N}(23)-\mathrm{H}(23) \ldots \mathrm{N}(16)$ & 0.8800 & 2.4400 & $2.802(4)$ & 105.00 \\
\hline $\mathrm{N}(23)-\mathrm{H}(23) \ldots \mathrm{O}(3) \# 2$ & 0.8800 & 2.0800 & $2.880(4)$ & 151.00 \\
\hline $\mathrm{N}(27)-\mathrm{H}(27) \ldots \mathrm{O}(15)$ & 0.8800 & 2.1100 & $2.964(4)$ & 164.00 \\
\hline $\mathrm{N}(27)-\mathrm{H}(27) \ldots \mathrm{N}(23)$ & 0.8800 & 2.3700 & $2.777(5)$ & 108.00 \\
\hline $\mathrm{C}(1)-\mathrm{H}(1 \mathrm{~B}) \ldots \mathrm{O}(26) \# 1$ & 0.9800 & 2.5000 & $3.365(5)$ & 147.00 \\
\hline $\mathrm{C}(11)-\mathrm{H}(11 \mathrm{C}) \ldots \mathrm{O}(12)$ & 0.9800 & 2.5900 & $3.018(5)$ & 106.00 \\
\hline $\mathrm{C}(17)-\mathrm{H}(17 \mathrm{~A}) \ldots \mathrm{O}(12)$ & 0.9900 & 2.5500 & $2.971(4)$ & 106.00 \\
\hline $\mathrm{C}(20)-\mathrm{H}(20) \ldots \mathrm{O}(22) \# 3$ & 1.0000 & 2.5200 & $3.099(5)$ & 117.00 \\
\hline $\mathrm{C}(28)-\mathrm{H}(28) \ldots \mathrm{O}(26)$ & 1.0000 & 2.3900 & $2.814(5)$ & 104.00 \\
& & & &
\end{tabular}




\section{Details of NMR titrations}

\section{Self association of Boc-MOPAS-OEt, 5.}

Chemical induced shift of $\mathbf{5}$ :

\begin{tabular}{c|c|c} 
concentration & \multicolumn{2}{|c}{$\delta[\mathrm{ppm}]$} \\
\hline$[\mathrm{mmol} / \mathrm{l}]$ & Py-NH & Amide-NH \\
\hline 124 & 9.176 & 5.095 \\
\hline 61.9 & 9.034 & 5.000 \\
\hline 43.4 & 8.980 & 4.962 \\
\hline 31.0 & 8.937 & 4.933 \\
\hline 19.1 & 8.889 & 4.892 \\
\hline 12.4 & 8.856 & 4.870 \\
\hline 8.26 & 8.836 & 4.857 \\
\hline 4.96 & 8.821 & 4.849 \\
\hline 3.18 & 8.809 & 4.845
\end{tabular}

HYP-NMR-output:

Refinement converged successfully

Chi-squared $=5.11$

sigma $=0.00257 \mathrm{rms}$ weighted residual $=0.0021871$

\begin{tabular}{l|c|c|c|c|c} 
& & Value & relative std devn & $\log$ beta & standard deviation \\
\hline Beta 2 & refined & $1.9821 \mathrm{E} 0$ & 0.0580 & 0.2971 & 0.0252
\end{tabular}



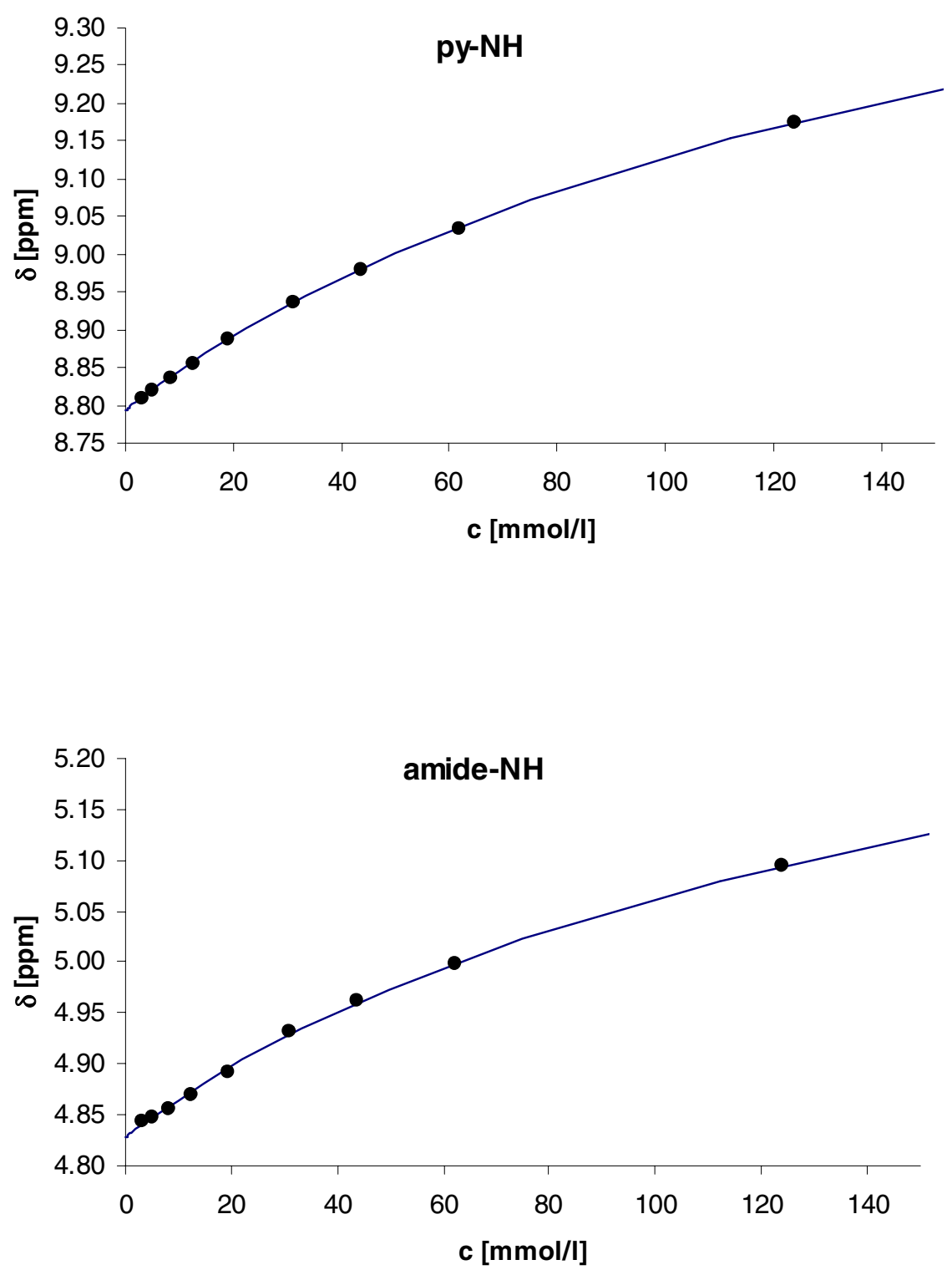
Titration of Ac-Val-Val-OMe (17) with Boc-MOPAS-OEt (5).

Chemical induced shift of $\mathbf{5}$ and 17:

\begin{tabular}{|c|c|c|c|c|c|}
\hline \multicolumn{2}{|c|}{ concentration $[\mathrm{mmol} / \mathrm{l}]$} & \multicolumn{4}{|c|}{$\delta[\mathrm{ppm}]$} \\
\hline & & Boc-M & PAS-OEt & Ac-Val- & al-OMe \\
\hline Boc-MOPAS-OEt & Ac-Val-Val-OMe & Py-NH & Boc-NH & Amid-NH 1 & Amid-NH 2 \\
\hline 40.0 & 121 & 9.6928 & 5.6018 & 7.23205 & 6.89615 \\
\hline 40.0 & 100 & 9.6411 & 5.5546 & 7.1777 & 6.85035 \\
\hline 40.0 & 80.0 & 9.5675 & 5.4902 & 7.09995 & 6.7845 \\
\hline 40.0 & 60.6 & 9.4915 & 5.4161 & 7.02265 & 6.7259 \\
\hline 40.0 & 47.1 & 9.4251 & 5.3551 & 6.9589 & 6.68095 \\
\hline 40.0 & 40.0 & 9.4110 & 5.3438 & 6.9426 & 6.8151 \\
\hline 40.0 & 34.0 & 9.3661 & 5.3052 & 6.9022 & 6.63845 \\
\hline 40.0 & 28.0 & 9.3261 & 5.2625 & 6.8668 & 6.61675 \\
\hline 40.0 & 24.0 & 9.2946 & 5.2328 & 6.83715 & 6.59715 \\
\hline 40.0 & 20.0 & 9.2635 & 5.2036 & 6.8134 & 6.58435 \\
\hline 40.0 & 18.0 & 9.2474 & 5.1874 & 6.79815 & 6.5746 \\
\hline 40.0 & 16.0 & 9.2266 & 5.1685 & 6.7852 & 6.56935 \\
\hline 40.0 & 13.2 & 9.2115 & 5.1525 & 6.76965 & 6.56115 \\
\hline 40.0 & 10.0 & 9.1802 & 5.1239 & 6.74465 & 6.54575 \\
\hline
\end{tabular}

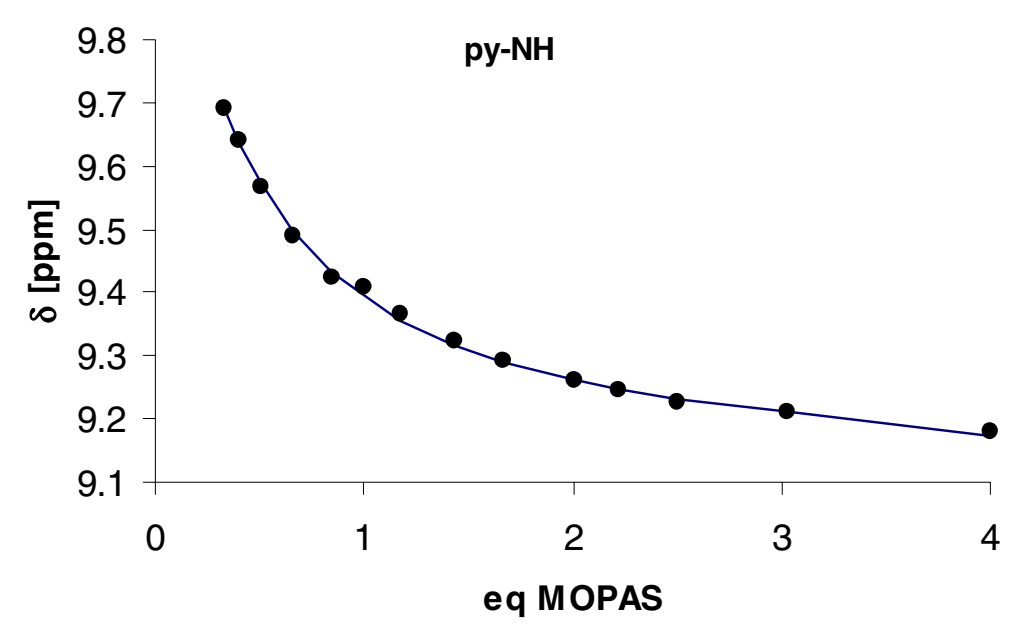




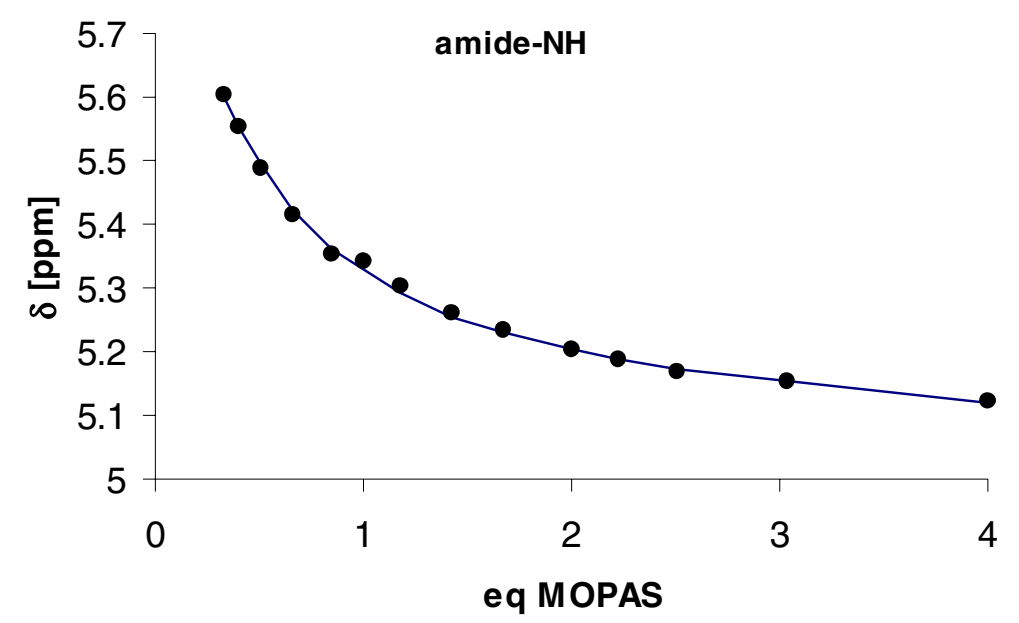

HYP-NMR output:

Refinement converged successfully

Chi-squared $=25.08$

sigma $=0.00640 \mathrm{rms}$ weighted residual $=0.0057542$

\begin{tabular}{l|c|c|c|c|c} 
& & Value & relative std devn & $\log$ beta & standard deviation \\
\hline Beta 1 & refined & $2.1360 \mathrm{E} 1$ & 0.0990 & 1.3296 & 0.0430 \\
\hline Beta 2 & constant & $1.9815 \mathrm{E} 0$ & & 0.2970 &
\end{tabular}


Self-association of Boc-(MOPAS $)_{2}$-OEt, 18.

Chemical induced shift of $\mathbf{1 8}$ :

\begin{tabular}{c|c|c|c} 
concentration & \multicolumn{3}{|c}{$\delta$ [ppm] } \\
\hline$[\mathrm{mmol} / \mathrm{l}]$ & py-NH1 & py-NH2 & amide-NH \\
\hline 74.0 & 10.680 & 10.410 & 5.510 \\
\hline 54.7 & 10.620 & 10.350 & 5.480 \\
\hline 37.3 & 10.540 & 10.270 & 5.440 \\
\hline 26.1 & 10.370 & 10.140 & 5.360 \\
\hline 14.8 & 10.310 & 10.060 & 5.330 \\
\hline 8.12 & 10.100 & 9.900 & 5.240 \\
\hline 4.06 & 9.840 & 9.720 & 5.140 \\
\hline 2.46 & 9.700 & 9.540 & 5.070 \\
\hline 1.41 & 9.480 & 9.430 & 5.000 \\
\hline 0.759 & 9.320 & 9.280 & 4.920
\end{tabular}

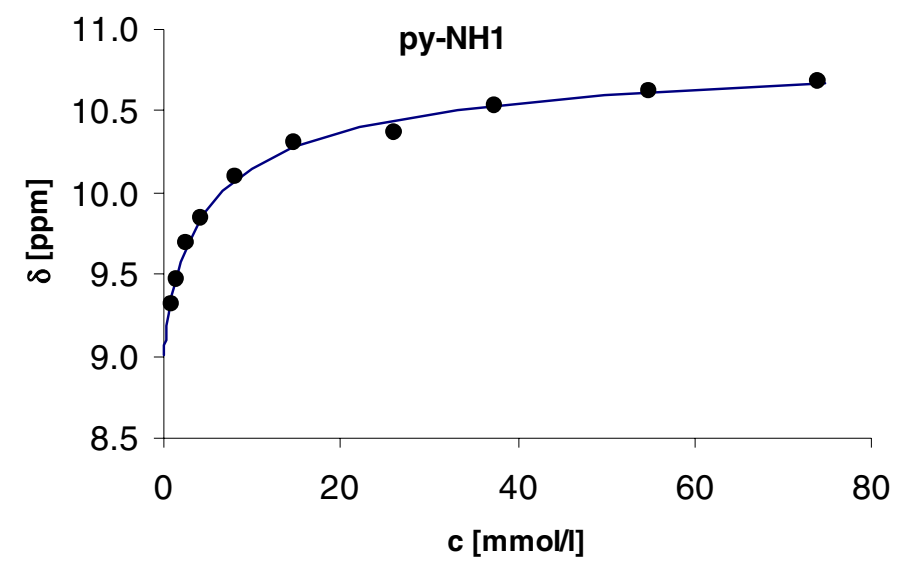



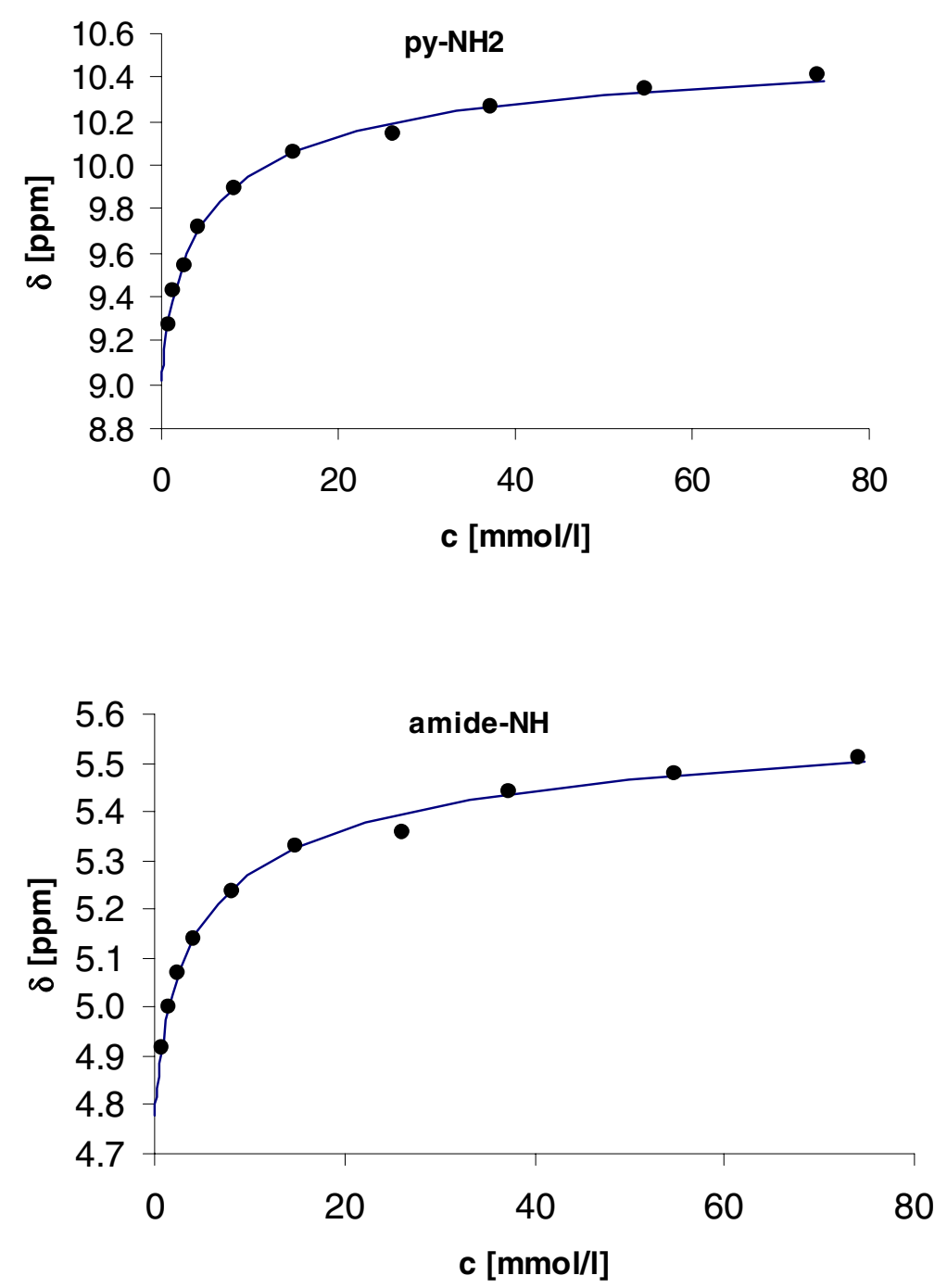

Hyp-NMR output:

Refinement converged successfully

Chi-squared $=12.67$

sigma $=0.02473 \mathrm{rms}$ weighted residual $=0.0216522$

\begin{tabular}{l|c|c|c|c|c} 
& & Value & relative std devn & $\log$ beta & standard deviation \\
\hline Beta 2 & refined & $1.3499 \mathrm{E} 2$ & 0.1143 & 2.1303 & 0.0496
\end{tabular}


Self-association of Boc-Phe-Ala-Val-Leu-OMe, 14.

Chemical induced shift of one selected amide-NH of 14:

\begin{tabular}{c|c} 
concentration & $\delta[\mathrm{ppm}]$ \\
\hline$[\mathrm{mmol} / \mathrm{l}]$ & amide-NH \\
\hline 0.400 & 4.784 \\
\hline 0.810 & 4.807 \\
\hline 1.18 & 4.814 \\
\hline 2.18 & 4.843 \\
\hline 3.15 & 4.866 \\
\hline 4.10 & 4.899 \\
\hline 5.02 & 4.931 \\
\hline 5.93 & 4.962 \\
\hline 6.81 & 4.987 \\
\hline 8.51 & 5.039 \\
\hline 10.1 & 5.092 \\
\hline 13.9 & 5.193 \\
\hline 18.5 & 5.292 \\
\hline 27.9 & 5.451 \\
\hline 37.0 & 5.569 \\
\hline
\end{tabular}

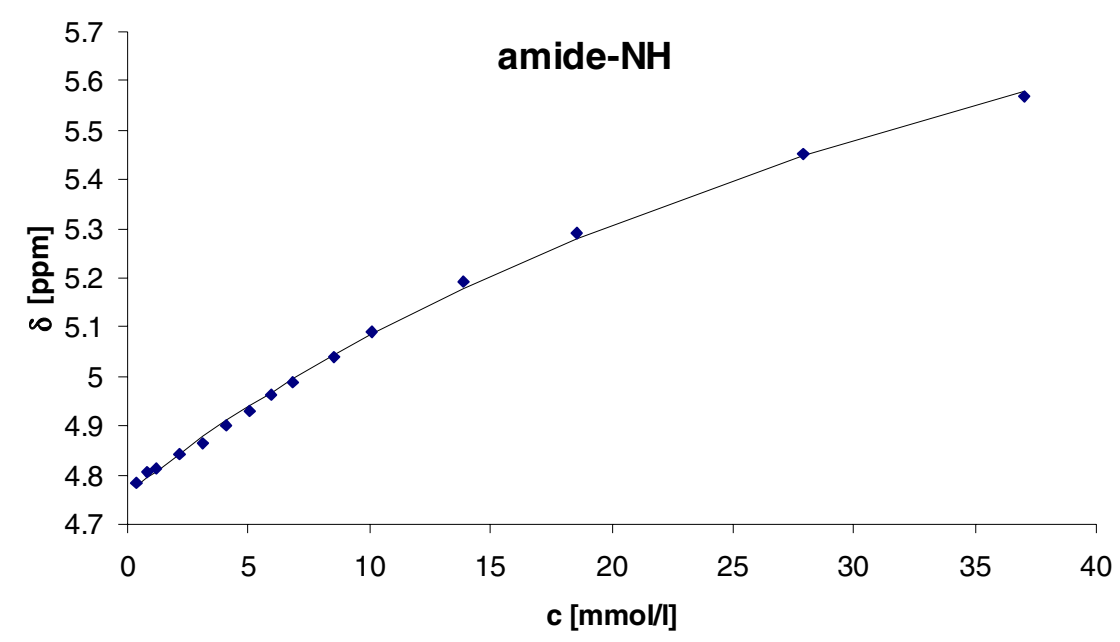


HypNMR-output:

Refinement converged successfully

Chi-squared $=10.07$

sigma $=3.02299 \mathrm{rms}$ weighted residual $=2.7038409$

\begin{tabular}{l|c|c|c|c|c} 
& & Value & relative std devn & $\log$ beta & standard deviation \\
\hline Beta 2 & refined & 6.2085E 0 & 0.1077 & 0.7930 & 0.0468
\end{tabular}

Titration of Boc-Phe-Ala-Val-Leu-OMe (14) with Boc-MOPAS-MOPAS-OEt (18).

Chemical induced shift of $\mathbf{1 8}$ :

\begin{tabular}{|c|c|c|c|}
\hline \multicolumn{2}{|c|}{ concentration $[\mathrm{mmol} / \mathrm{l}]$} & \multicolumn{2}{|c|}{$\delta[\mathrm{ppm}]$} \\
\hline $\begin{array}{l}\text { Boc-MOPAS- } \\
\text { MOPAS-OEt }\end{array}$ & $\begin{array}{l}\text { Boc-Phe-Ala- } \\
\text { Val-Leu-OMe }\end{array}$ & py-NH1 & py-NH2 \\
\hline 6.67 & 11.0 & 10.048 & 9.835 \\
\hline 8.48 & 10.5 & 10.097 & 9.878 \\
\hline 10.7 & 10.0 & 10.147 & 9.922 \\
\hline 13.2 & 9.38 & 10.191 & 9.960 \\
\hline 16.1 & 8.66 & 10.241 & 10.005 \\
\hline 19.4 & 7.87 & 10.287 & 10.048 \\
\hline 22.9 & 7.01 & 10.328 & 10.086 \\
\hline 26.5 & 6.14 & 10.362 & 10.120 \\
\hline 30.1 & 5.26 & 10.393 & 10.151 \\
\hline 33.5 & 4.43 & 10.421 & 10.178 \\
\hline 36.6 & 3.66 & 10.444 & 10.201 \\
\hline 2.25 & 7.37 & 9.796 & 9.591 \\
\hline 3.06 & 7.15 & 9.837 & 9.628 \\
\hline 3.82 & 6.94 & 9.875 & 9.663 \\
\hline 4.71 & 6.70 & 9.918 & 9.701 \\
\hline
\end{tabular}




\begin{tabular}{l|l|l|l}
6.17 & 6.30 & 9.978 & 9.756 \\
\hline 7.86 & 5.84 & 10.040 & 9.813 \\
\hline 9.88 & 5.29 & 10.102 & 9.869 \\
\hline 12.2 & 4.67 & 10.158 & 9.920 \\
\hline 14.6 & 4.01 & 10.209 & 9.966 \\
\hline 17.1 & 3.34 & 10.251 & 10.005 \\
\hline 19.4 & 2.71 & 10.306 & 10.054 \\
\hline 21.2 & 2.21 & 10.323 & 10.072
\end{tabular}
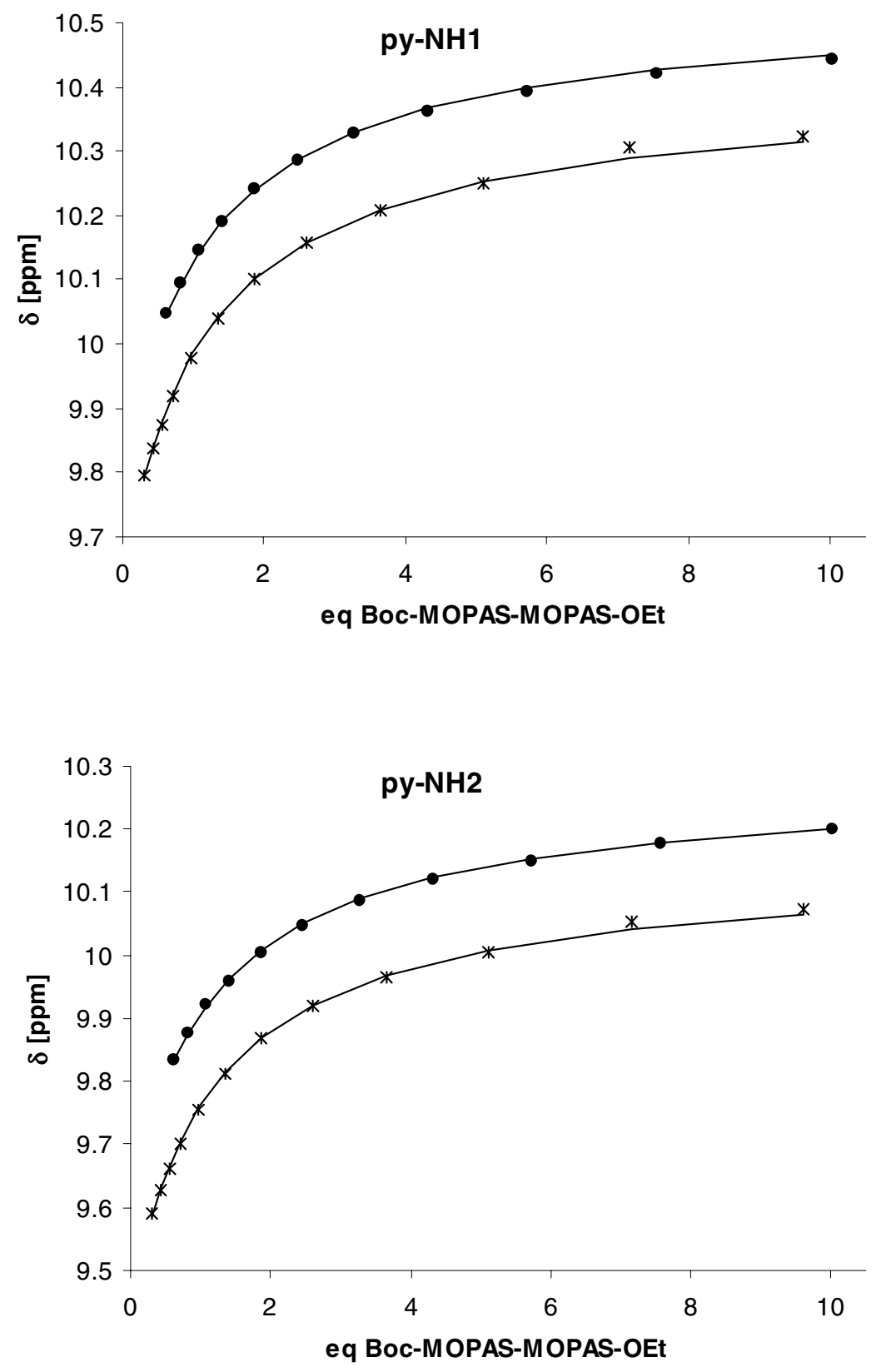


\section{HypNMR-output:}

Refinement converged successfully

Chi-squared $=7.22$

sigma $=0.00388 \mathrm{rms}$ weighted residual $=0.0035263$

\begin{tabular}{c|c|c|c|c|c} 
& & Value & relative std devn & log beta & standard deviation \\
\hline Beta 2:0 & refined & $5.7286 \mathrm{E} \mathrm{2}$ & 0.3013 & 2.7580 & 0.1309 \\
\hline Beta 1:1 & refined & $3.3914 \mathrm{E} \mathrm{2}$ & 0.2090 & 2.5304 & 0.0908 \\
\hline Beta 0:2 & constant & $6.2087 \mathrm{E} \mathrm{0}$ & & 0.7930 &
\end{tabular}




\section{NMR data for structure determination}

$\beta$-turn $10\left(\mathrm{CDCl}_{3}, 75.3 \mathrm{mM}\right)$
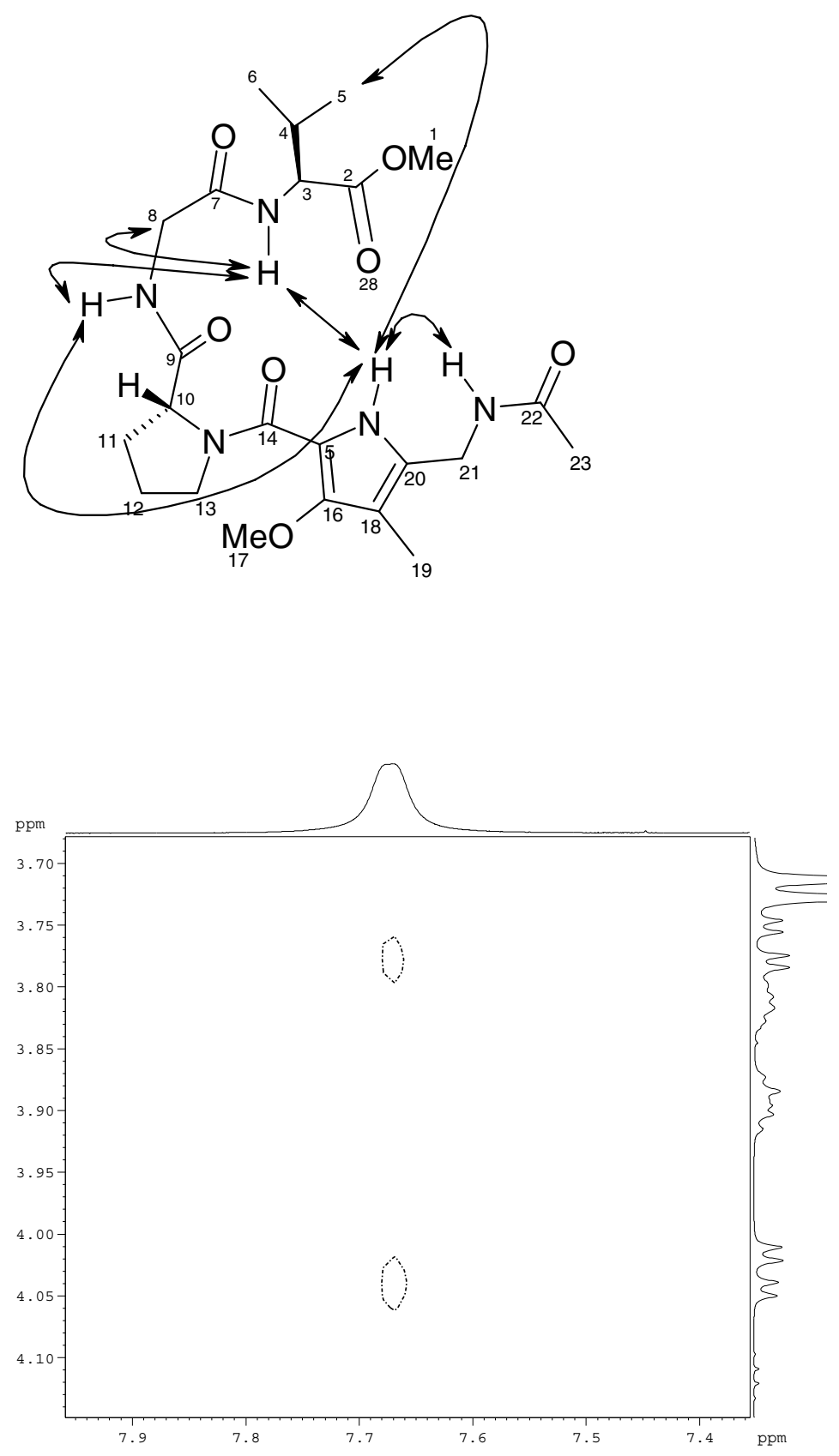

ROE: 4-NH - 8- $\mathrm{H}_{\mathrm{a} / \mathrm{b}}$. Intensity ratio $=1.13 \rightarrow$ distance ratio $=1.02$ 


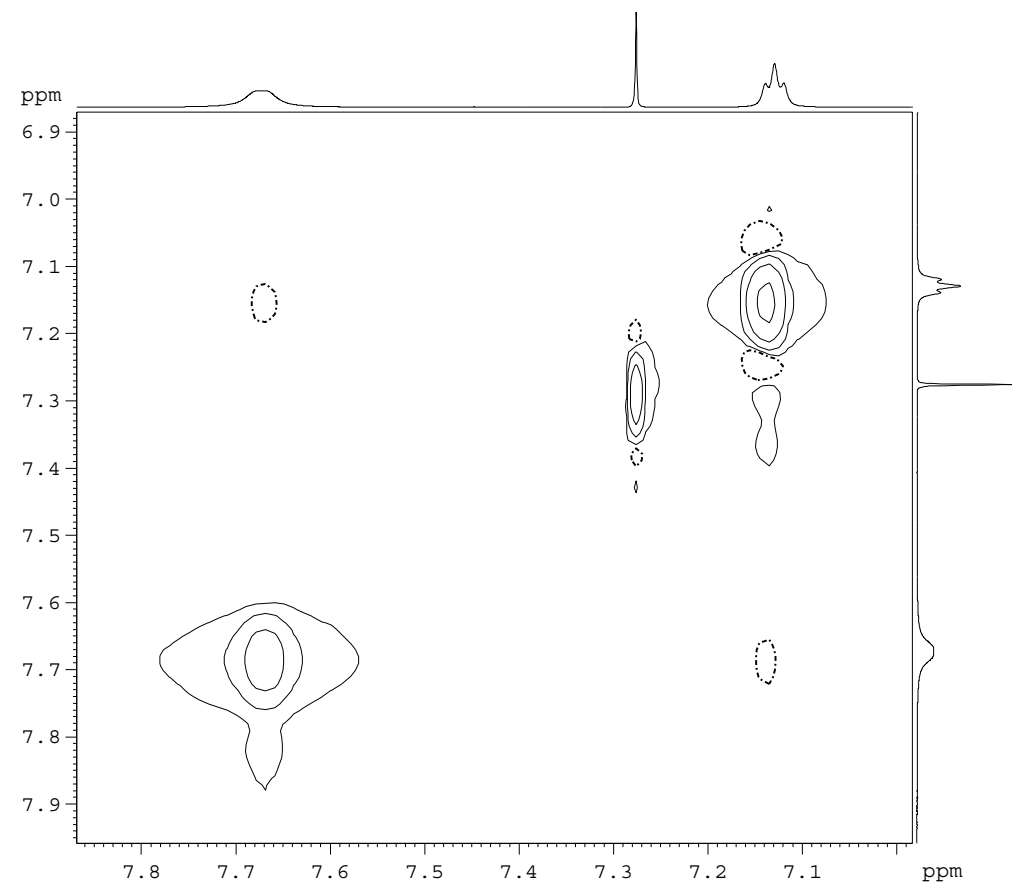

ROE: 3-NH - 8-NH

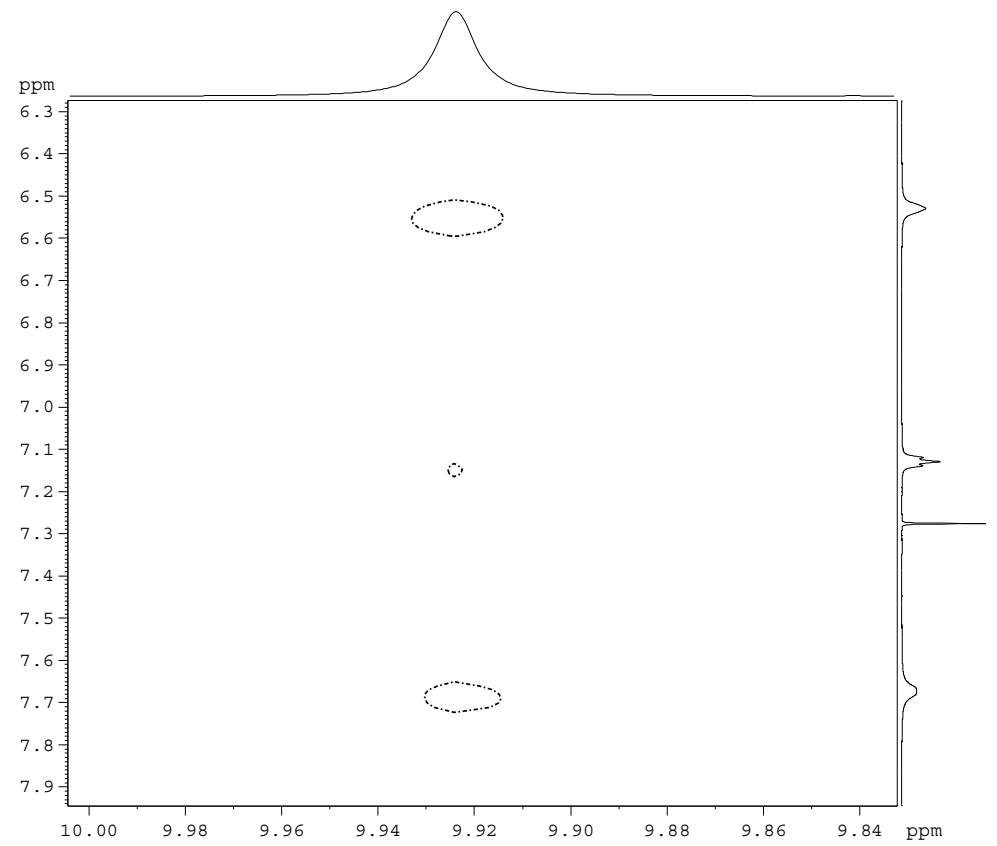

ROE: 15-NH - 3-NH (upper signal)

- 8-NH (middle signal)

- 21-NH (lower signal) 


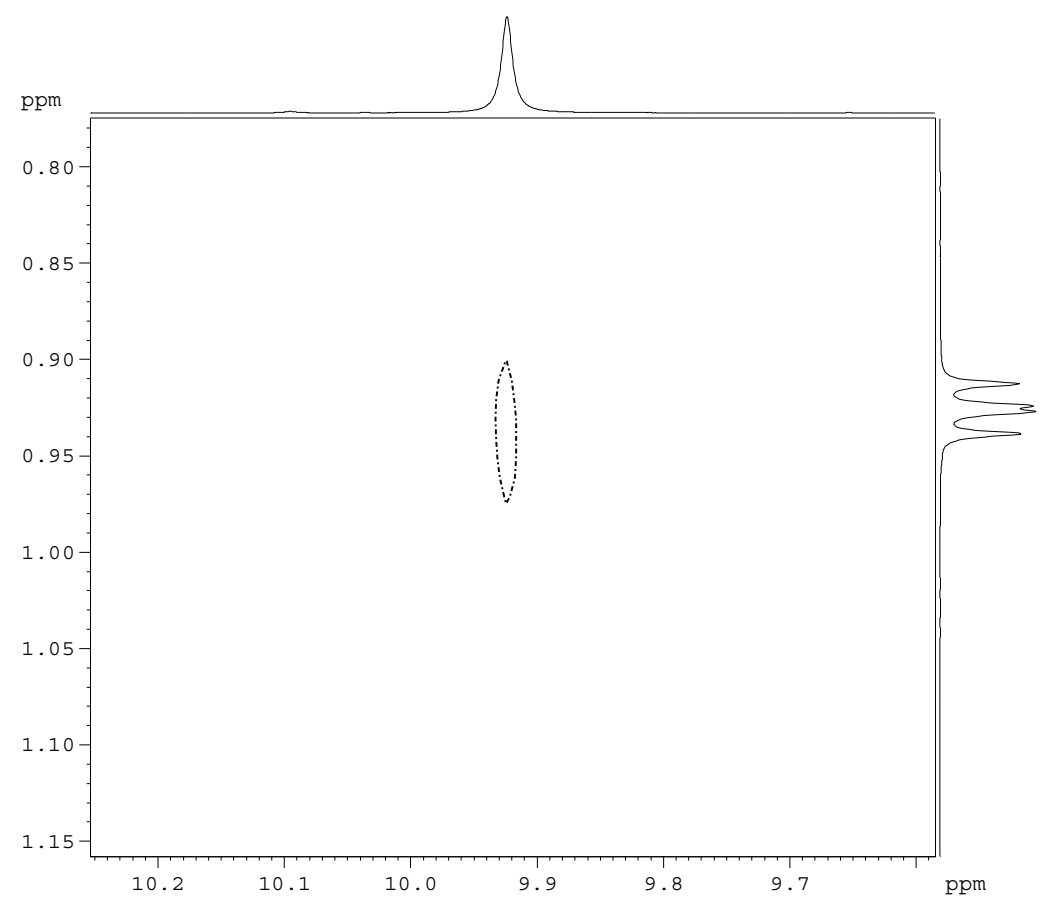

ROE: $15-\mathrm{NH}-5-\mathrm{H}$

$\beta$-turn $16\left(\mathrm{CDCl}_{3}, 47.7 \mathrm{mM}\right)$

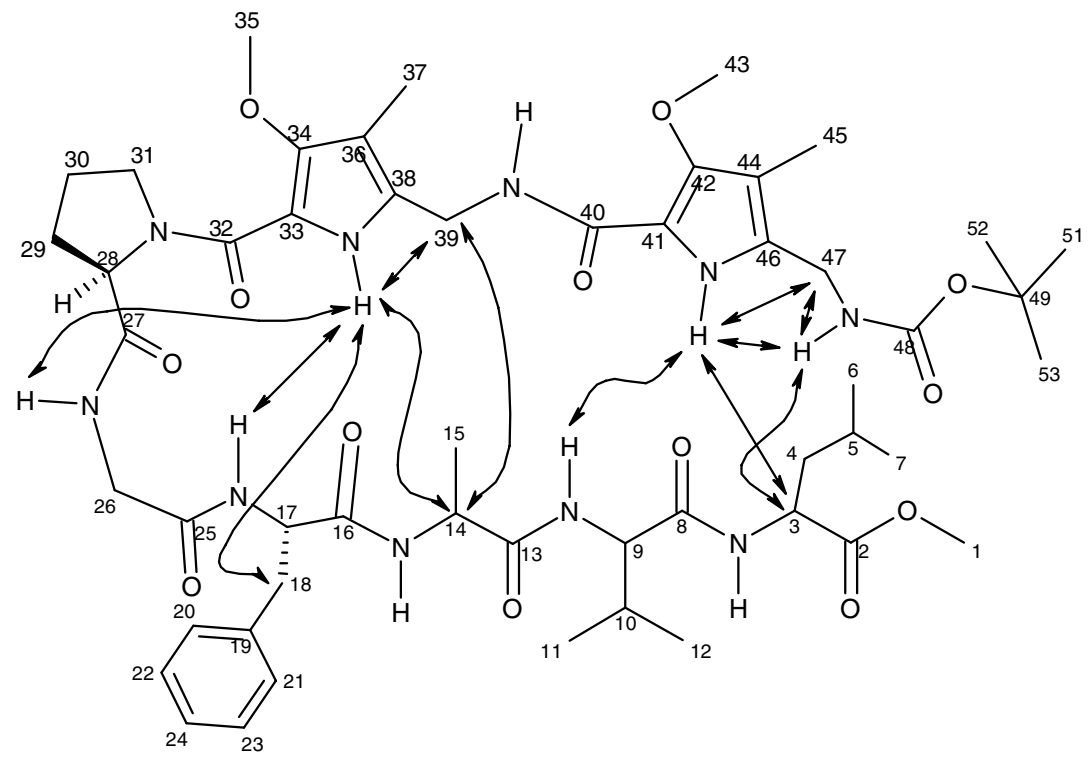




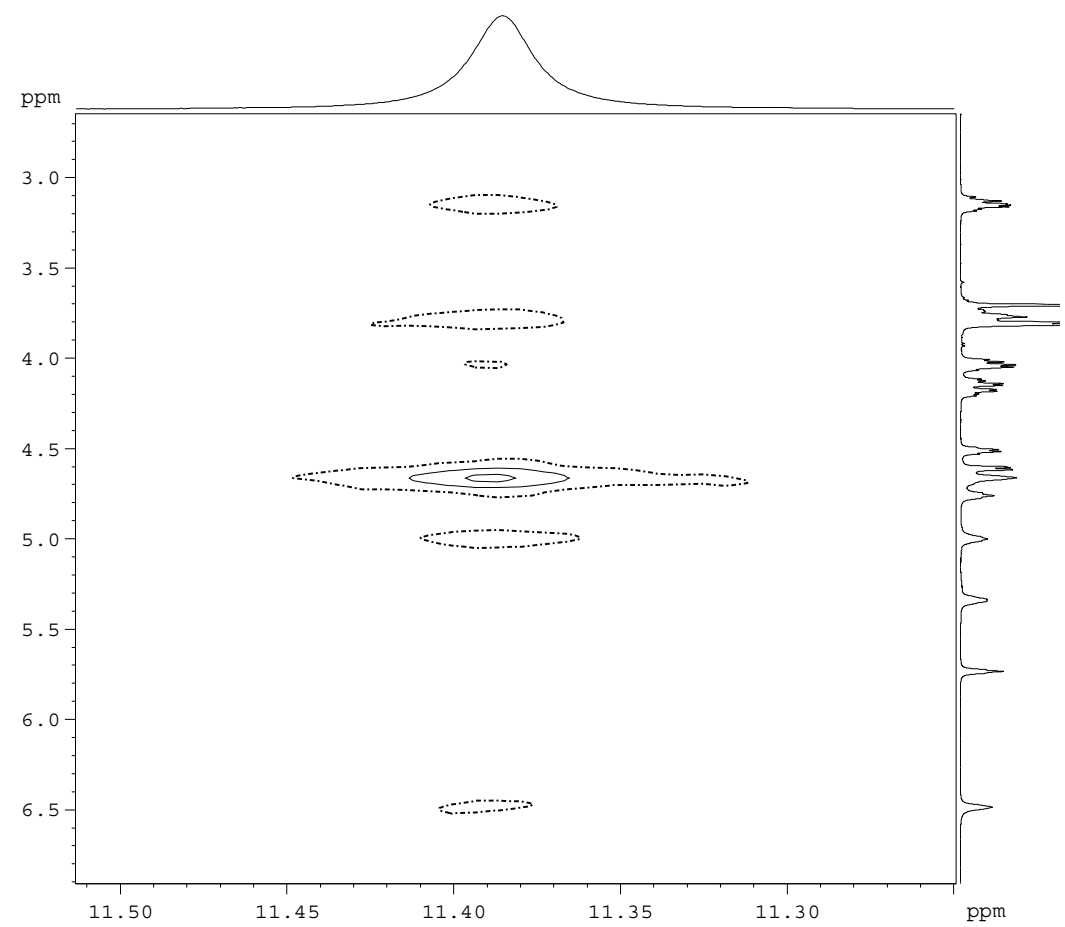

ROE: 33-NH - 18-H (upper signal)

- 39-H ( $4^{\text {th }}$ signal $)$

- $14-\mathrm{H}\left(5^{\text {th }}\right.$ signal $)$

- 26-NH (lower signal) 


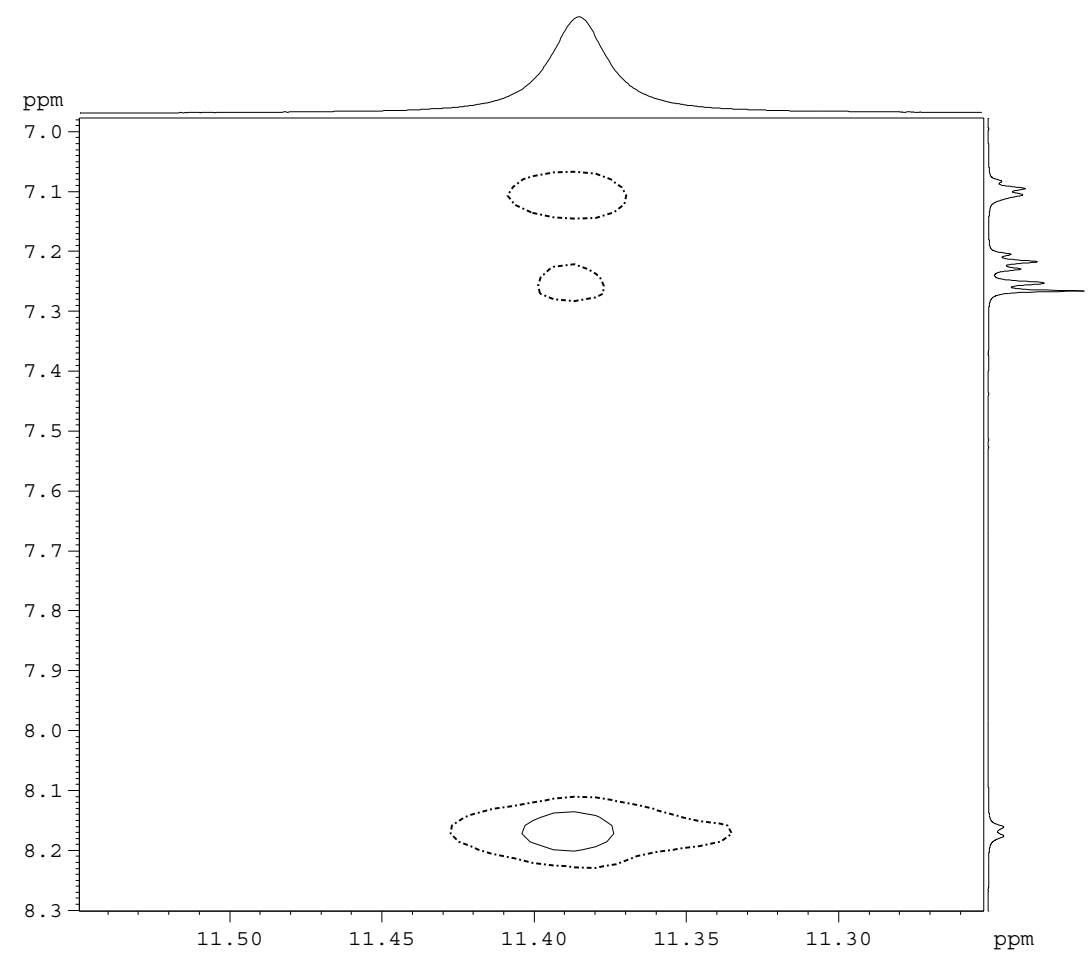

ROE: 33-NH - phenyl-protons overlapped with 39-NH (upper signal)

- phenyl-protons (middle signal)

- 17-NH (lower signal)

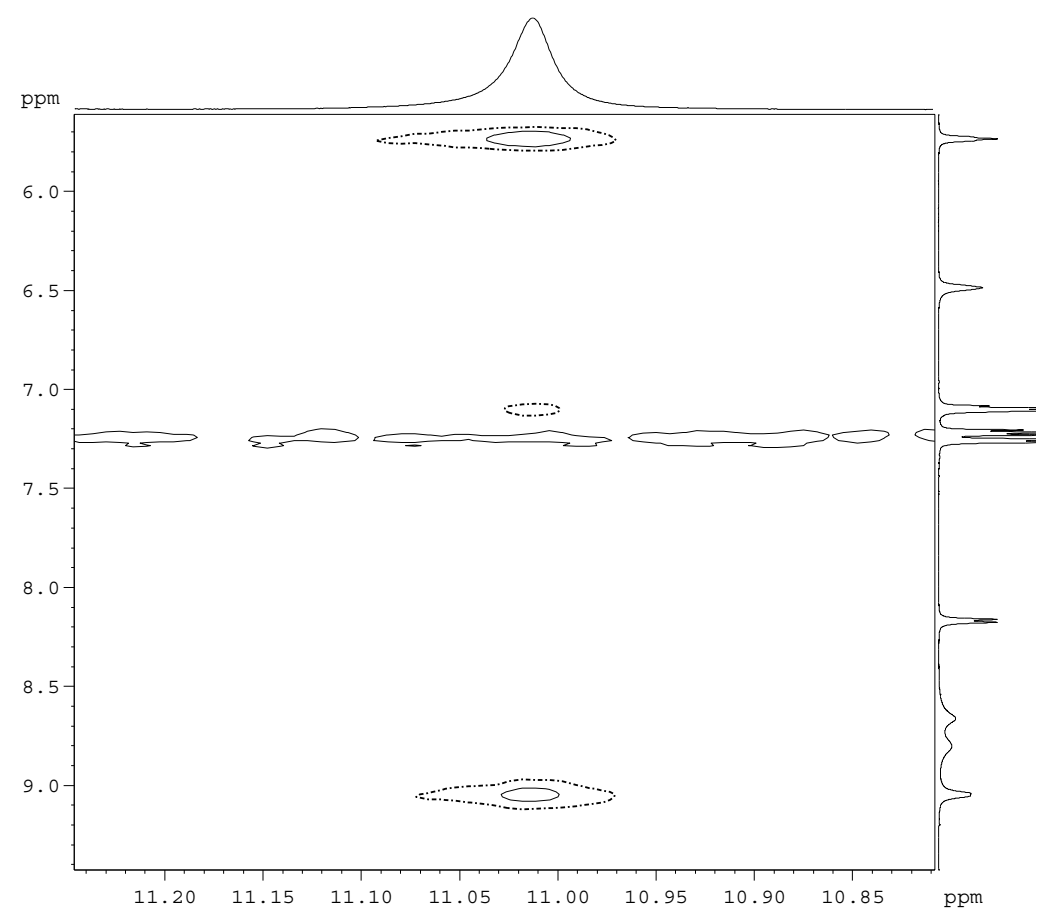

ROE: 41-NH - 47-NH (upper signal) - 9-NH (lower signal) 


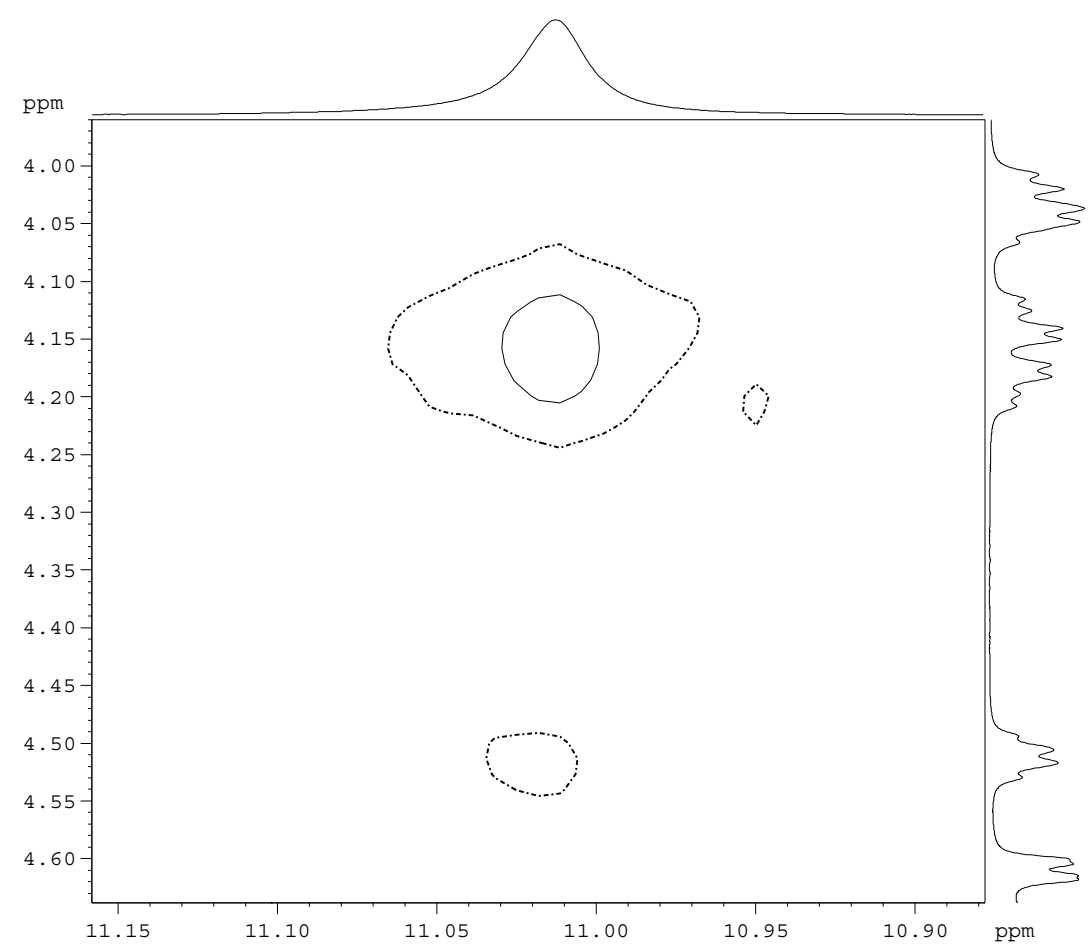

ROE: 41-NH - 47-H

$-3-\mathrm{H}$

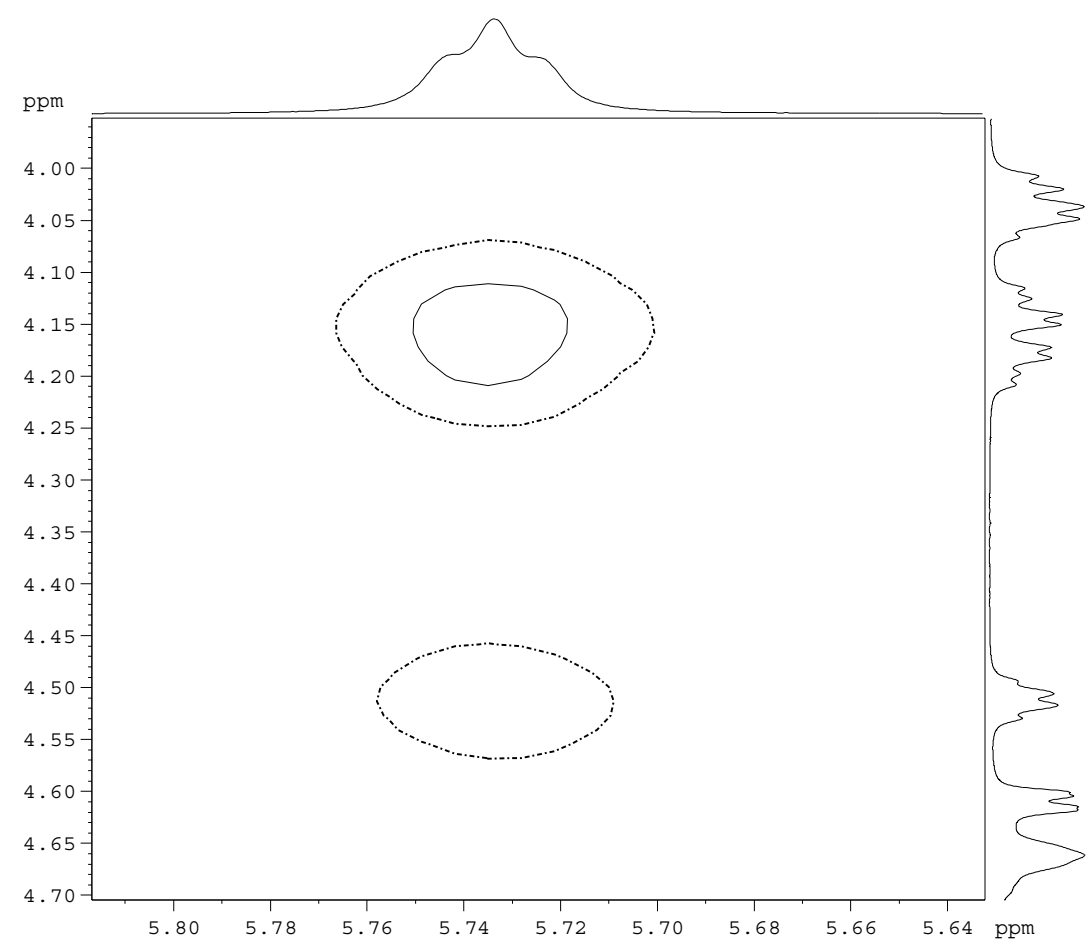

ROE: 47-NH - 47-H

- 3-H 


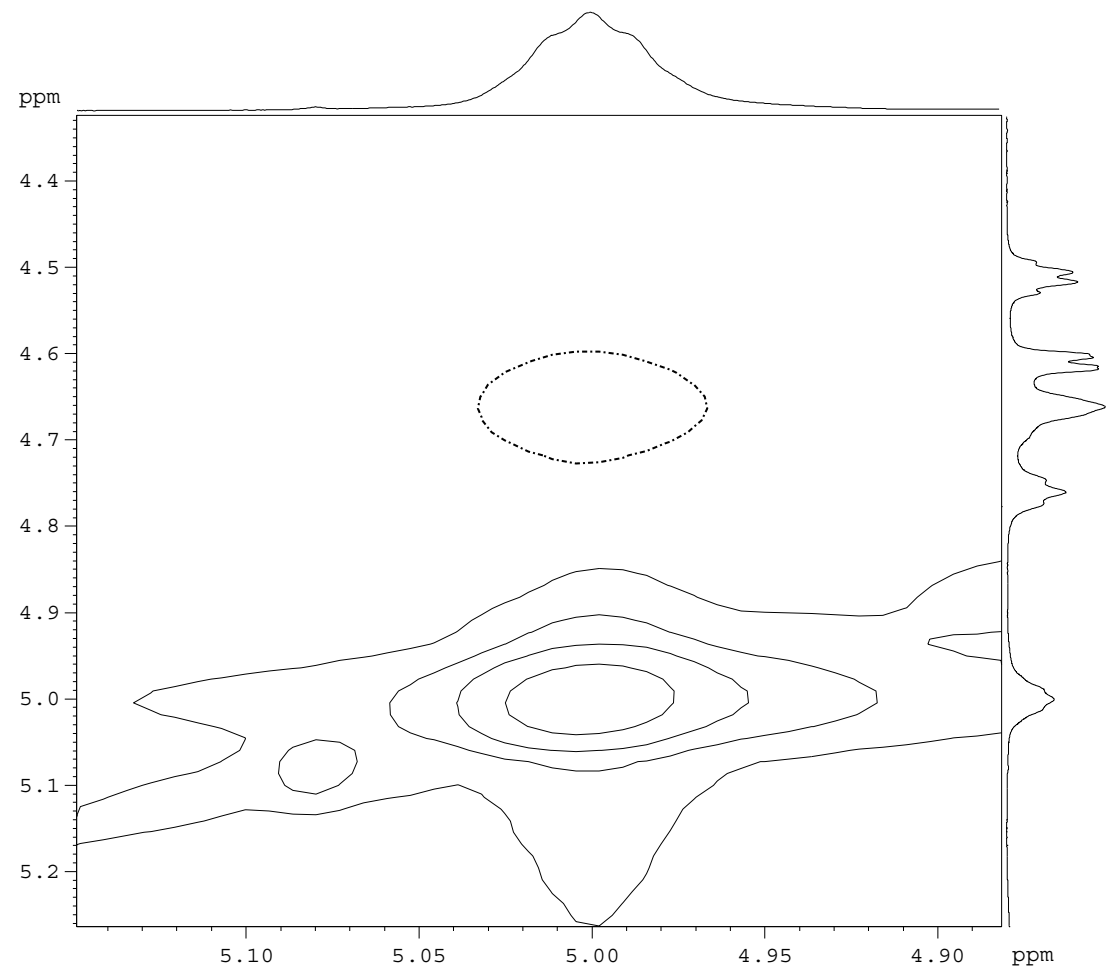

ROE: $39 \mathrm{H}-14 \mathrm{H}$

No significant change in chemical shifts was observed at different concentrations for both $\beta$ turn structures. This indicates that the compounds show no intermolecular association under the conditions of the experiments. 
${ }^{\mathbf{1}} \mathbf{H}-\mathrm{NMR}$ of compound $4(250 \mathrm{MHz}, \mathrm{CDCl} 3)$ :

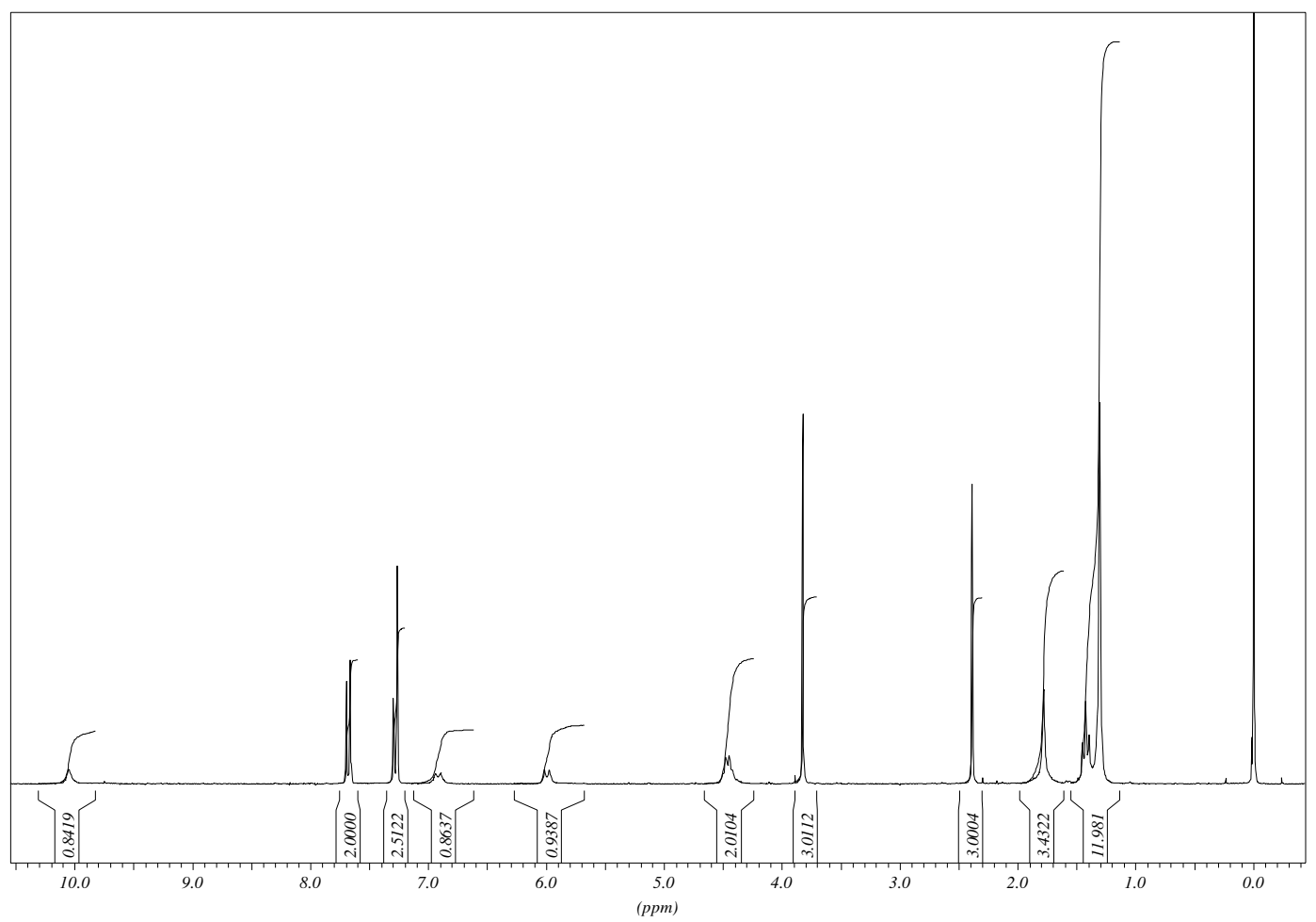

${ }^{\mathbf{1}} \mathbf{H}$-NMR of compound $7(300 \mathrm{MHz}, \mathrm{CDCl} 3)$ :

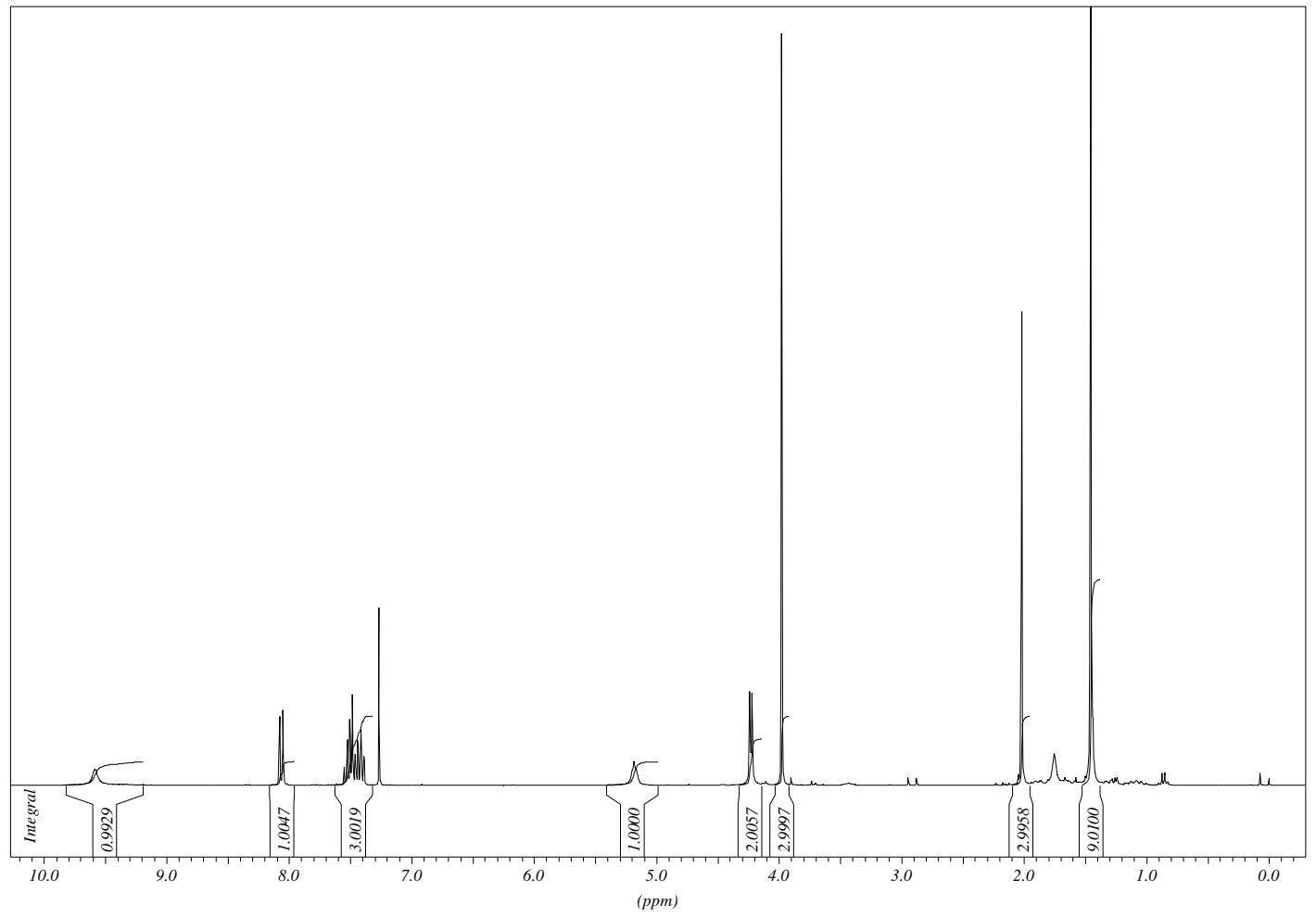


${ }^{1}$ H-NMR of compound 13 (300 MHz, DMSO-d $\mathrm{d}_{6}$ ):

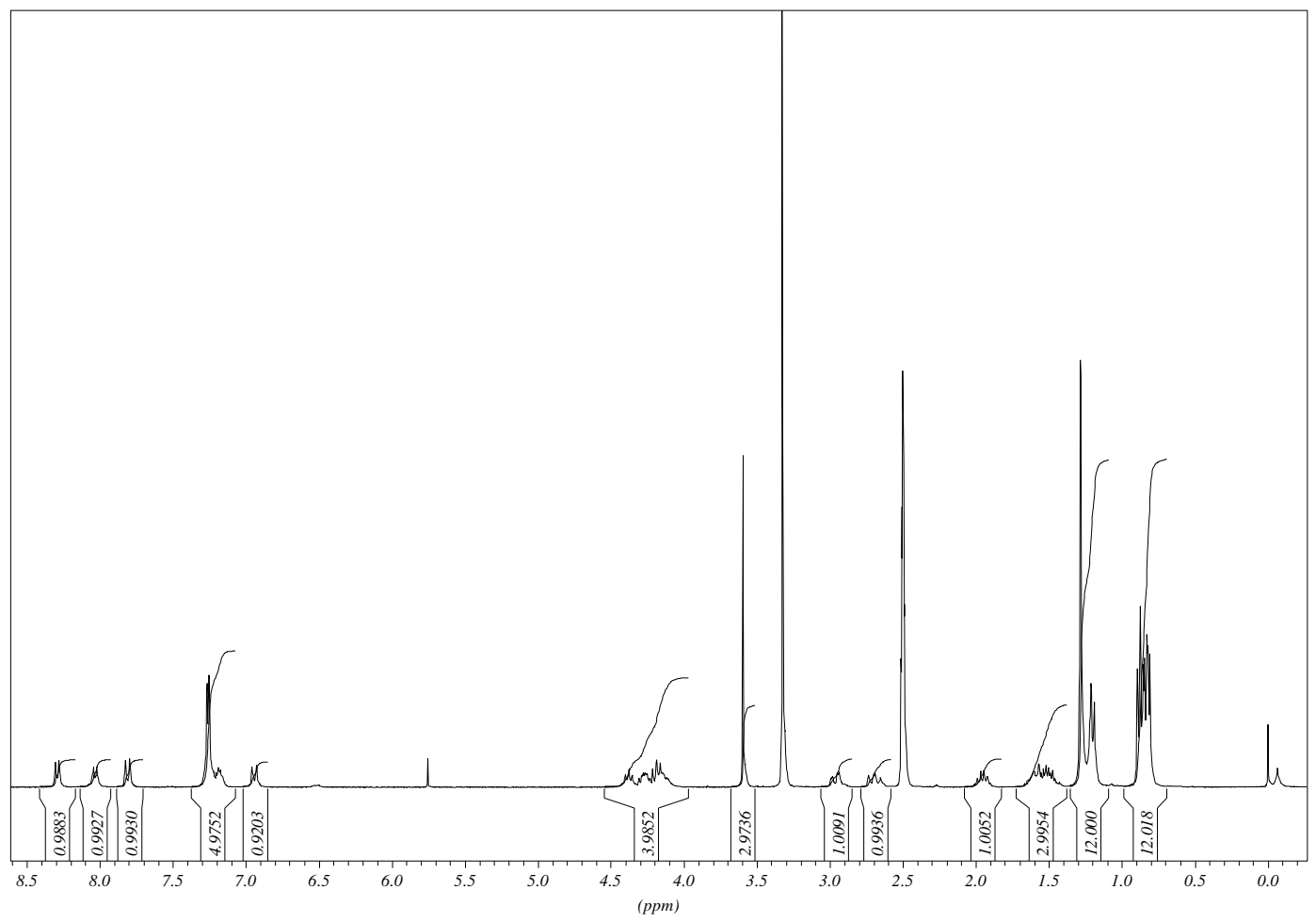

${ }^{1}$ H-NMR of compound 15 (300 MHz, DMSO-d ${ }_{6}$ ):

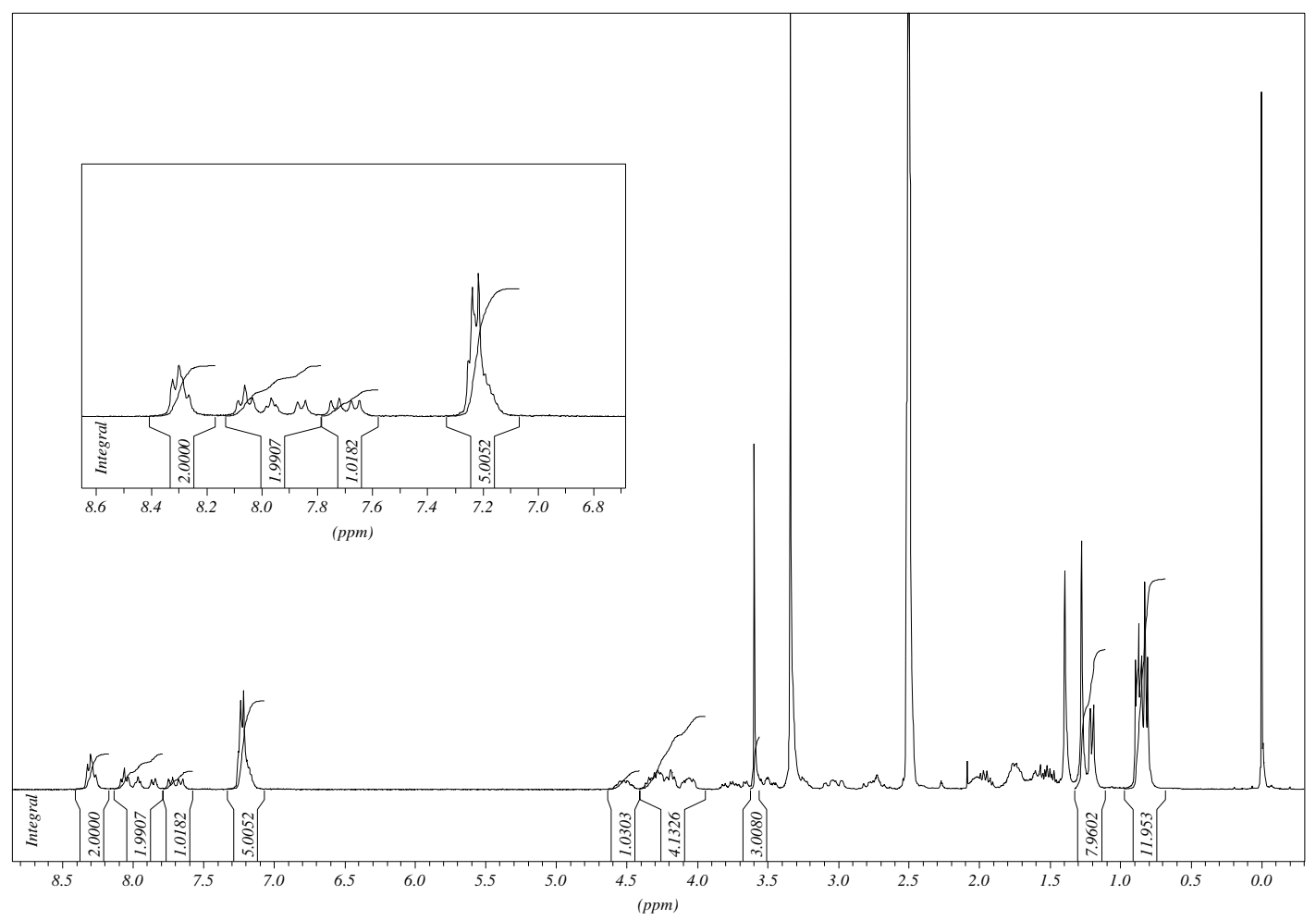

